\title{
NANOSCALE SCHOTTKY BARRIER VISUALIZATION UTILIZING COMPUTATIONAL MODELING AND BALLISTIC ELECTRON EMISSION MICROSCOPY
}

\section{Westly Nolting}

\author{
A Thesis \\ Submitted to the State University of New York, Polytechnic Institute \\ in Partial Fulfillment of \\ the Requirement for the Degree of \\ DOCTOR OF PHILOSOPHY
}

Colleges of Nanoscale Science and Engineering 2018 


\section{NANOSCALE SCHOTTKY BARRIER VISUALIZATION UTILIZING COMPUTATIONAL MODELING AND BALLISTIC ELECTRON EMISSION MICROSCOPY}

Westly Nolting

(C) Copyright 2018

All Rights Reserved 


\section{CONTENTS}

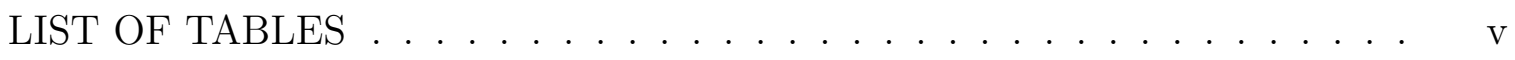

LIST OF FIGURES . . . . . . . . . . . . . . . . . . . vi

ACKNOWLEDGMENTS ........................ xi

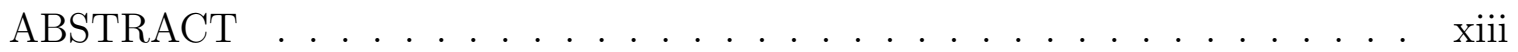

1. INTRODUCTION AND LITERATURE REVIEW . . . . . . . . . . . . . 1

1.1 Introduction . . . . . . . . . . . . . . . . . 1

1.2 Literature Review . . . . . . . . . . . . . . . . . . 2

1.2.1 Metal-Semiconductor Junctions . . . . . . . . . . . . . 3

1.2.2 Ballistic Electron Emission Microscopy . . . . . . . . . . 5

1.3 Thesis Overview . . . . . . . . . . . . . . . . . 11

2. Theory of BEEM and Related Phenomena . . . . . . . . . . . 13

2.1 Scanning Tunneling Microscopy . . . . . . . . . . . . . . . 13

2.2 Schottky Barrier Formation . . . . . . . . . . . . . . . 17

2.2.1 Image Force Lowering . . . . . . . . . . . . . . . . . . . . . 23

2.3 Ballistic Electron Emission Microscopy . . . . . . . . . . . . . . 24

2.3.1 BEEM Spectra Modelling . . . . . . . . . . . . . 24

2.3.2 BEEM Spectra Fitting . . . . . . . . . . . . 26

2.4 Electron Scattering . . . . . . . . . . . . . . . . . . . 28

2.4.1 Elastic Scattering . . . . . . . . . . . . . . 28

2.4 .2 Inelastic Scattering . . . . . . . . . . . . . . 32

2.5 Modelling . . . . . . . . . . . . . . . . 35

2.5.1 Monte-Carlo Modelling . . . . . . . . . . . . 35

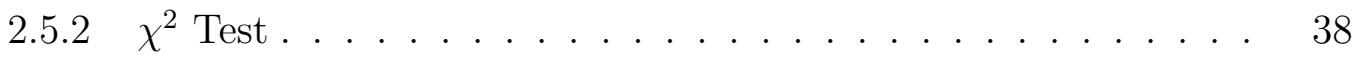

2.5.3 Model Algorithm .................. . 40

3. EXPERIMENTAL METHODS . . . . . . . . . . . . . . . . 42

3.1 Sample Preparation . . . . . . . . . . . . . . . . . . 42

3.1.1 Substrate preparation ................ 42

3.1.2 Metal depositions . . . . . . . . . . . . . . . 43

3.1.3 Sample mounting . . . . . . . . . . . . . . 44 
3.2 Sample Measurements . . . . . . . . . . . . . . . . . . . 45

3.2.1 Current-Voltage Spectroscopy _ . . . . . . . . . . . . 45

3.2.2 Ballistic Electron Emission Microscopy _ . . . . . . . . . . 46

3.2 .3 BEEM Mapping. . . . . . . . . . . . . . . . . . 48

4. Nanoscale Schottky barrier mapping of thermally evaporated and sputter deposited $\mathrm{W} / \mathrm{Si}(001)$ diodes and the presence of multiple Schottky barriers 51

4.0 .1 Results . . . . . . . . . . . . . . . . . 51

4.0 .2 Discussion . . . . . . . . . . . . . . . . . . 53

4.1 Multiple Barrier Height Modelling . . . . . . . . . . . . . . . . 60

5. Study of Chromium Thickness and Silicide Formation Study . . . . . . . 65

5.1 Detection of Silicide Formation in $\mathrm{Cr} / \mathrm{Si}(001) \ldots \ldots \ldots$

5.1 .1 Results . . . . . . . . . . . . . . . . . . 65

$5.1 .2 \quad$ Discussion . . . . . . . . . . . . . . . . . . 68

5.2 Cr Thickness Study . . . . . . . . . . . . . . . . 76

5.2 .1 Fermi level pinning . . . . . . . . . . . . . 76

$5.2 .2 \quad \mathrm{Si}(100)$ Results $\ldots \ldots \ldots \ldots \ldots \ldots$

$5.2 .3 \quad \mathrm{Si}(111)$ Results . . . . . . . . . . . . . . . . . . 79

5.2 .4 Discussion . . . . . . . . . . . . . . . . . . . . . . 81

6. Conclusions and Future Directions . . . . . . . . . . . . . . 86

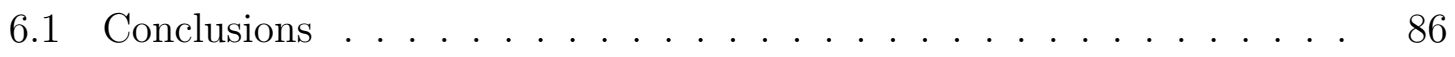

6.2 Future Studies . . . . . . . . . . . . . . . . . . . . . . . 87

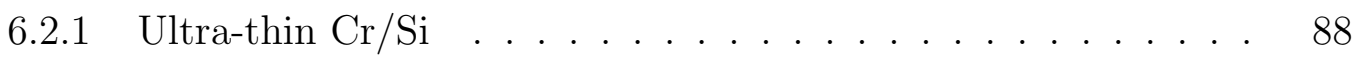

6.2.2 Thickness dependent Scatter . . . . . . . . . . . . . . 88

LITERATURE CITED . . . . . . . . . . . . . . . . . . . . . . . 90 


\section{LIST OF TABLES}

2.1 $\chi^{2}$ distribution table. DoF are the number of degrees of freedom assumed and $\mathrm{P}$ value is the probability associated with the $\chi^{2}$ value. . . . . . . 40

3.1 Temperatures are measured in degrees $\mathrm{C}$ and rate is calculated in nanometers per minute (nm/min). Metals used for deposition are all $99.99 \%$ pure and bought commercially. . . . . . . . . . . . . . . . .

5.1 Values utilized in the modeling of the BEEM threshold distributions displayed in Fig. 5.6 \& Fig. 5.7. . . . . . . . . . . . . . . . . . . . . . . . 


\section{LIST OF FIGURES}

1.1 A "cat's whisker" device used in early radio broadcasting for creating point-contact rectifying diodes of a metal wire and semiconducting crystal to prevent current flow in a specific direction. . . . . . . . . . . . .

1.2 Diagram of BEEM measurement schematic. Electrons can have 4 different paths to overcoming the barrier: (1) ballistic transport, (2) inelastic scattering in the metal, (3) elastic scattering from grain boundaries, interface reflection, or selective scattering at the interface, (4) inelastic impact ionization . . . . . . . . . . . . . . . . . .

2.1 Tunnel bands for electron tunneling through a potential barrier. . . . . 14

2.2 STM tunnel bands for electron tunneling through a potential barrier with an applied bias. Tunnel probability is controlled by Fowler-Nordheim tunneling. . . . . . . . . . . . . . . . . . .

2.3 The formation of the Schottky barrier comes in 3 parts. (a) The metal and semiconductor surfaces are brought in contact with each other. (b) The electrons in the conduction band of the semiconductor flow towards the interface. (c) The conduction and valence bands bend in response to flow of charges to the interface and a Schottky barrier forms. . . . . .

2.4 The Schottky-Mott relation does not follow reported measured data. Schottky-Mott relation is plotted for GaAs along with various metal Schottky barriers on GaAs. Linear fit to the data is also plotted to show disagreement with Schottky-Mott relation . . . . . . . . . .

2.5 (a) The induced charge density from $\mathrm{Nb}$ dopants in the $\mathrm{TiO}_{2}$ lattice. (b) Band bending with the vertical bar indicating the depth location of the Nb dopant atom. . . . . . . . . . . . . . . . .

2.6 Typical BEEM spectrum for fitting routine. The LSB and FSB are chosen and maximizing the $R^{2}$ is done until a barrier height is found with the best fit to the data. . . . . . . . . . . . . . .

2.7 Total momentum representation as parallel and perpendicular momentum with respect to the interface of the metal and semiconductor. . . .

2.8 Acceptance cone of scattered angles as transmitted energy across the Schottky barrier with respect to the energy of the electron and the interface of the metal and semiconductor. . . . . . . . . . . . . . . 
2.9 Examples of the energy distribution of electrons in the metal for large tip biases on the left column and the corresponding spectrum from a BEEM measurement on the right column. (i) The distribution of electrons at the interface for very thin metal film layers with all ballistic electron transport, (ii) electrons are inelastically scattered with thicker metal films and the start of secondary electron contributing to BEEM current, (iii) strong inelastic scattering that attenuates the signal and secondary electrons accounting for larger portion of BEEM current. Shaded blue region is contributions from secondary electrons $[1] . \ldots . . . . . . .$.

2.10 Scattering results based off of Fermi-liquid theory and the relation to the attenuation length. Example values are used to show comparison. . . .

2.11 Computational modelling results and the effect of charge density. An increase in donor density decreases the barrier height. Units of charge are measured with depth from the interface in e/nm. Schottky maps are shown with each histogram to demonstrate visual representation of the barrier height changing. . . . . . . . . . . . . . . . .

2.12 Computational modelling results and the effect of multiple barrier heights. 36

2.13 Computational modelling results and the effect of elastic and inelastic scattering. The elastic modelling shows a high energy tail appear while the inelastic modelling will have an overall large decrease in counts. $\Gamma_{e}$ is the elastic scattering probability and $t$ is an inelastic scattering length.

3.1 Diagram showing the silicon surface a) before exposure to oxygen, b) after $\mathrm{SiO} 2$ formation on the surface, and c) after hydrogen passivation of the surface with $\mathrm{HF}$ etch of the native oxide. . . . . . . . . . . . . .

3.2 Diagram showing the wiring of a ballistic electron emission microscopy (BEEM) plate. Metal and semiconductor both have separate contacts with preamplifier reading current from the semiconductor with bias placed on the tip. . . . . . . . . . . . . . . . . . .

3.3 Diagram of the wiring for BEEM setup. Both the Metal and semiconductor are grounded; with a preamplifier reading current from the semiconductor while a bias placed on the tip to the metal. . . . . . . . . .

3.4 Energy band diagram of the BEEM setup, showing the STM tip on the left, metal film in the center and the n-type semiconductor on the right.

3.5 Energy band diagram of the BHEM setup, showing the STM tip on the left, metal film in the center and the p-type semiconductor on the right.

3.6 BEEM Mapping grid. 10000 spectra are taken over a $1 \mu \mathrm{m}^{2}$ area region. The region is broken down into 25 individual frames with data taken every $10 \mathrm{~nm}$. . . . . . . . . . . . . . . . . . . 
4.1 TEM image of sputter deposited tungsten/Si sample. An interfacial layer of tungsten and tungsten silicide is formed. . . . . . . . . . . . .

4.2 (a) Linearized fit of BHEM spectra of p-type sputter Schottky diode using exponent of $n=2.5$. (b) n-type linearized fit of sputter Schottky BEEM spectra. (c) p-type e-beam linearized fit of BHEM spectra showing an increased Schottky barrier height compared to its sputter equivalent. (d) n-type e-beam linearized fit of BEEM spectra. . . . . . . . . . .

4.3 Average spectra of all four samples plotted together to demonstrate shift in barrier heights and agreement to the band gap of silicon. Each curve is plotted versus percent transmission of the BEEM current. . . . . . .

4.4 Each map displayed is representative of the electrostatic interface. (a) and (b) BHEM and BEEM measurements show a uniform interface for sputter. (c) and (d) e-beam samples show a very disordered interface caused by a change in the interface chemistry during deposition. . . . .

4.5 Histogram of Schottky barrier heights from fits to the individual spectra. (a) \& (b) are for the sputter sample and show a symmetric and narrow Gaussian-like distribution and good agreement between the mean Schottky and fit to the average. (c) \& (d) are for the e-beam sample showing a skewed asymmetric distribution attributed to elastic scattering of the hot electrons. . . . . . . . . . . . . . . .

4.6 Tungsten-Silicon Schottky maps and distributions show the difference between an incomplete silicide formation in (a), and a uniform $\mathrm{W} / \mathrm{Si}$ interface in (b). The Schottky maps for each show the distribution of threshold values over a $1 \mu m^{2} \ldots \ldots \ldots \ldots \ldots$

4.7 $\quad R^{2}$ vs Schottky barrier height for all samples. In the case of sputter (a) and (b), the Schottky barrier heights fits are associated with a tight distribution around the average $R^{2}$ value. Both (c) and (d) e-beam samples exhibit a broad distribution of $R^{2}$ values which correspond to a wider range of Schottky barrier heights. . . . . . . . . . . . . . .

4.8 The effects of model parameters and number of barrier heights. The progression of $\chi^{2}$ and scattering rate as parameters are added are shown.

4.9 Tungsten-Si computational modeling confidence levels from P-value tests using the incomplete gamma function. Best fits are obtained when modeling reaches a $3 \sigma$ value for $\mathrm{P}$-value tests. $\mathrm{x}$-axis values correspond to modeling shown in Fig.4.8. . . . . . . . . . . . . . .

$4.10(6.5 \mathrm{~nm}) \mathrm{Au} /(30 \mathrm{~nm}) \mathrm{Ag}$ is show in (a) with the majority of threshold values pinned to a higher energy than the barrier height of $\mathrm{Ag} / \mathrm{Si}$. (10nm) $\mathrm{Au} /(10 \mathrm{~nm}) \mathrm{Ag}$ is shown in (b) with the majority of threshold values equal to the barrier height of $\mathrm{Au} / \mathrm{Si} . \ldots \ldots \ldots \ldots$ 
5.1 Self aligning silicide process contains three major steps: Metallization of the transistor finFET, annealing to promote diffusion of the metal and silicon to grow a silicide, and selective etching of the left-over metal. . .

5.2 (a) Linearized fit of BHEM spectra of p-type Cr/Si Schottky diode using exponent of $n=2$. (b) n-type linearized fit of Schottky BEEM spectra. (c) BHEM and BEEM Schottky indicating the summation of the barrier heights. (inset) (d) Bright field TEM image of the $\mathrm{Au} / \mathrm{Cr} / \mathrm{Si}(001)$ interface and (e) corresponding EDS map. . . . . . . . . . . . . . . .

5.3 Transmission electron microscopy imaging of $\mathrm{Au}(8) / \mathrm{Cr}(2) / \mathrm{Si}(001)$. (a) Bright field image of $\mathrm{Cr} / \mathrm{Si}(001)$ Schottky diode and (b) EDS imaging of $\mathrm{Au}-\mathrm{Cr}$ layers. . . . . . . . . . . . . . . . . . . .

5.4 (a) $\mathrm{Cr} / \mathrm{p}-\mathrm{Si}(001)$ which shows a higher order degree of variation in the threshold values of the spectra at each tip location. (b) $\mathrm{Cr} / \mathrm{p}-\mathrm{Si}(001)$ Schottky map showing very little skewing to higher energies. . . . . . .

5.5 Spectra threshold distributions for (a) p-type and (b) n-type $\mathrm{Cr} / \mathrm{Si}(001)$ Schottky diode with associated $R^{2}$ values beneath. Inset is the map of the thresholds over a $1 \mu \mathrm{m}$ squared area. The lines plotted are fits to a lognormal probability distribution function. . . . . . . . . . . .

5.6 (a) $\mathrm{Cr} / \mathrm{n}-\mathrm{Si}(001)$ modelled histogram and data showing skewing to higher energies and degree of agreement between the two. (b) Improved agreement when a second threshold is added to the simulation with a mixture factor of $0.9 \ldots \ldots \ldots \ldots \ldots \ldots$

5.7 (a) $\mathrm{Cr} / \mathrm{p}-\mathrm{Si}(001)$ modelled histogram and data showing skewing to higher energies and degree of agreement between the two. (b) Improved agreement when a second threshold is added to the simulation with a mixture factor of 0.5 and a reduced $\Gamma_{e}$ of $6 \%$. . . . . . . . . . . .

5.8 (a) $\mathrm{Cr} / \mathrm{p}-\mathrm{Si}(001)$ slope map which shows a higher order degree of variation in the slopes of the spectra at each tip location. (b) $\mathrm{Cr} / \mathrm{n}-\mathrm{Si}(001)$ slope map with a consistent value across the micron area. . . . . . . . . . .

5.9 (a) $\mathrm{Cr} / \mathrm{p}-\mathrm{Si}(001) R^{2}$ scatter plots of the goodness of fit and the associated threshold value which shows a higher order degree of variation in fit. (b) $\mathrm{Cr} / \mathrm{n}-\mathrm{Si}(001)$ localized to very good fitting parameters near a value of 1 .

5.10 All average spectra for each of the different thicknesses are shown. An increase in $I_{t i p}$ is for increasing the percentage transmission being collected as BEEM current. . . . . . . . . . . . . . . . . . . 
5.11 (a)(b)(c) Linear fits to the average spectra, (d)(e)(f) show histogram plots of the measured threshold values of each spectra. Each histogram gives indication of the elastic and inelastic scattering in the metal as well variation in the threshold at the interface., (g)(h)(i) show threshold barrier height maps corresponding to thickness for chromium. (a) (d) (g) refer to $1.16 \mathrm{~nm}$ thickness, (b)(e)(h) refer to $1.35 \mathrm{~nm}$, and (c)(f)(i) refer

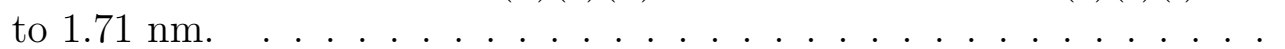

5.12 The scatter plot of the goodness of fit $R^{2}$. Tighter distributions generate more gaussian like histogram distribution of barrier heights. (a) to (c) is increasing in thickness from $1.16 \mathrm{~nm}$ to $1.35 \mathrm{~nm}$ to $1.71 \mathrm{~nm} . \quad \ldots$. . .

5.13 Average spectra for each of the different thicknesses are shown. An increase in $I_{t i p}$ is for increasing the percentage transmission being collected as BEEM current. . . . . . . . . . . . . . . . . . . .

5.14 (a)(b)(c) Linear fits to the average spectra, (d)(e)(f) show histogram plots of the measured threshold values of each spectra. Each histogram gives indication of the elastic and inelastic scattering in the metal as well variation in the threshold at the interface., $(\mathrm{g})(\mathrm{h})(\mathrm{i})$ show threshold barrier height maps corresponding to thickness for chromium. (a) (d) (g) refer to $1.26 \mathrm{~nm}$ thickness, (b)(e)(h) refer to $1.65 \mathrm{~nm}$, and (c)(f)(i) refer to $1.91 \mathrm{~nm} . \ldots \ldots \ldots \ldots \ldots \ldots$

5.15 The scatter plot of the goodness of fit $R^{2}$. Tighter distributions generate more gaussian like histogram distribution of barrier heights. (a) to (c) is in increasing in thickness $1.26 \mathrm{~nm}, 1.65 \mathrm{~nm}, 1.91 \mathrm{~nm} . \ldots \ldots$. . . .

5.16 Trend of the barrier height as thickness increases. $\mathrm{Si}(100)$ and $\mathrm{Si}(111)$ both follow a similar downward slope followed by a leveling off at the barrier height of Cr. . . . . . . . . . . . . . . .

6.1 Trend of the barrier height as thickness increases. $\mathrm{Cu} / \mathrm{Si}(001)$ shows an increasing trend of scattering and attenuation of hot electrons. . . . . . 


\section{ACKNOWLEDGMENTS}

The work presented here and discussions argued are comprised of help, discussion,

and support from many others whose words of encouragement or directly helped in measurements. I give out heartfelt thanks to all of those who contributed but there are a few people who I would like to thank in particular.

I would first like to thank my co-workers and fellow Ph.D candidates in the LaBella lab: Dr. Chris Durcan, Dr. Avyaya Jayanthinarasimham, Dr. Akitomo Matsubayashi, and Dr. Robert Balsano. Each of them helped me learn, progress, and achieve everything I wanted to when I started working in the lab. They spent countless hours helping me with my work and without their input and discussions this work would have been not nearly as complete. I would like to thank the past students that, while did not directly give input, helped develop the measurement techniques and foundations of knowledge in the lab into what it was for my use.

I would like to also thank my wonderful girlfriend, Stacey, for all of the love, support, and patience I needed to complete this program.

To all my friends and fellow CNSE students who helped me feel supported and encouraged the belief that the trials and endeavours needed to complete a Ph.D were worth it, thank you. Specifically, Dr. Brian McGowan for all of his help while a student at CNSE.

My undergraduate university physics department and advisor, Dr. Kevin Stokes, provided the knowledge and framework for me to succeed as a researcher in the field of physics, condensed matter, and material science, as well as allowing me to work in his lab and really learn how physics research is conducted.

I would like to thank my committee members: Dr. Carl Ventrice, Dr. Alain Diebold, Dr. James Lloyd, and Dr. Steve Consiglio.

And finally, most of all, I would like to thank my advisor Dr. Vincent LaBella. His guidance, teaching, and support allowed me to pursue research in areas which I did not realize would be interesting and fruitful. I would like to thank him for allowing me to travel to present my work at conferences and experience the physics community, and for helping me develop the language and style needed to successfully 
publish in physics.

This work was supported by the National Science Foundation (DMR-123456) and the Semiconductor Research Corporation, Center for Advanced Interconnect Science and Technology. 


\begin{abstract}
Understanding the properties and performance of semiconductor interfaces on the nanoscale advances semiconductor device technology which has had tremendous impact on nearly all aspects of our daily lives. Investigating the nanoscale fluctuations in the electrostatics of metal-semiconductor, or Schottky, interfaces is crucial. However, techniques for directly measuring the electrostatics at an interface are limited. Current state-of-the-art finFETs use metal-semiconductor silicides, such as Ti-Si/Si, for Schottky source-drain contacts. Studying the underlying physics of the Schottky barrier interface of silicides and other metal-semiconductor systems is critical for measuring the Schottky barrier accurately, which can be accomplished with ballistic electron emission microscopy (BEEM), a scanning tunneling microscopy (STM) based technique.

In this work, the visualization of the interface to nanoscale dimensions is enhanced by computational modelling of threshold histograms acquired by the BEEM measurement technique. Modelling using a kinetic Monte-Carlo approach is utilized to simulate the distributions of barrier heights that includes effects from the interface and transport of the hot electrons as well as indication of a multi-barrier heights present at the interface. The aid of this modelling enables the discovery of several underlying properties of the interface. Analyzing the parameters of the modelling and comparing to measured data provides detailed insight into the effects that both electron scattering and incomplete silicide formation in $\mathrm{W} / \mathrm{Si}(001)$ and $\mathrm{WSi}_{2} / \mathrm{Si}(001)$ have upon the transport of electrons through these structures, which is difficult to detect with conventional current-voltage measurements. The modelling also includes simulation of multiple barriers present at the interface due to the intermixing of similar metals such as $\mathrm{Au}$ and $\mathrm{Ag}$ at the interface of $\mathrm{Si}(001)$ In this regard, Schottky barrier visualization as the combination of histograms, mapping, and modelling provides a new insight into the local nanoscale phenomenon of the Schottky barrier. This thesis investigates the modelling of these metal-semiconductor systems and uses modelling to look at metal thickness dependent effects on the Schottky barrier from Fermi-level pinning in $\mathrm{Au} / \mathrm{Cr}-\mathrm{Si} / \mathrm{Si}(001)$ and $\mathrm{Au} / \mathrm{Cr}-\mathrm{Si} / \mathrm{Si}(111)$ silicide.
\end{abstract}




\section{CHAPTER 1 INTRODUCTION AND LITERATURE REVIEW}

\section{$1.1 \quad$ Introduction}

Semiconductors are the bedrock of all modern innovation in the twentieth and twenty first century. Nearly all aspects of the daily life are enhanced by technology developed using semiconductors. From the simplest technologies like light emitting diodes (LEDs) and solar cells to advanced technologies such as self-driving cars and quantum computers, all of these devices have semiconductors as one of their fundamental components of operation and design. We live our lives in luxury compared to the times before microprocessors, touch screens, electric cars, solar energy, and personal computers were available. Semiconductors have given us the power to see galaxies and star formations billions of light years away and also have given us the foundation for developing the nanoscale electrical devices for use in all aspects of modern society [2].

For a semiconductor device to operate there must be a pathway of conducting material to deliver electric charge efficiently. Copper is the choice conducting material used in the industry for metallic interconnects to deliver proper voltages to all the devices in a chip [3]. The interconnect pathways come in contact with the semiconductor devices and a metal-semiconductor junction is formed. This junction is often referred to as the Schottky contact or the Schottky barrier, coined from its namesake, Walter H. Schottky. The quintessential property of the Schottky barrier is the formation of a potential barrier at the interface of the two materials and current behaves as a rectifier when a voltage is applied across the barrier.

The properties and underlying physical phenomena that govern the Schottky barrier is still a topic that has unsolved properties since its discovery [4]. While a plethora of information is known about the Schottky barrier, the complex nature of all materials, charge transport, diffusion, density of states and scattering make the problem of studying the Schottky barrier challenging. Methods to measure the Schottky barrier such as current-voltage and capacitance-voltage techniques can extrapolate the barrier height but direct measurement of the Schottky barrier is elu- 
sive with these conventional measurement tools. Much of the discussion about the nature of the Schottky barrier in literature is derived by modelling the Schottky barrier $[5,6,7,8,9,10]$.

Modeling of physical mechanisms has provided tremendous improvement in benchmarking, characterization, fabrication, data analysis, parameter extrapolation, and process simulation and control [2]. These areas include developing predictive models for: comprehensive methods for novel devices to increase performance, decrease power usage, and improve yield and manufacturability; first principles modeling of defect and trap states in novel materials; multi-scale modeling of novel material properties for device designs including charge, carrier mobility, ionic transport, band gaps, crystalline structures, etc. The metal/semiconductor junction is one of the areas in semiconductor research that benefits from optimization of materials and electrical transport and modeling of these two properties gives insight into changes that would improve industry advancements in technology, such as detection and measurement of silicides, multiple Schottky barrier heights, and charge density fluctuations at the interface.

Ballistic electron emission microscopy (BEEM) is a technique which provides direct visualization of electrostatics for metal/semiconductor junctions [11]. BEEM is an STM technique that images and measures the potential energy of a buried Schottky barrier interface to nanoscale resolution by utilizing the atomic positioning capability of the STM tip. In BEEM, a voltage bias is applied between the STM tip and metal which allows electrons to tunnel through vacuum into the metal. Electrons with enough forward momentum to exceed the Schottky barrier are collected on the backside of the semiconductor as BEEM current. As the voltage is swept, a spectra is collected and fit to extract the Schottky barrier height.

\subsection{Literature Review}

The following chapter will be focused on a literature review of BEEM and related topics. At first some overview of the Schottky barrier and some of its history and then an extensive review of the initial BEEM publications that first probed BEEM measurements and capabilities of the technique in imaging and measuring the electrostatics of an interface. Related phenomena will be looked at next such as 
elastic scattering of electrons, attenuation length, BEEM transmission, and dopant density affects on the barrier height.

\subsubsection{Metal-Semiconductor Junctions}

Ferdinand Braun first posited the properties of the metal-semiconductor junction in 1874 when he used mercury to make a contact between copper sulfide and iron sulfide. He noticed an assymetrical response to the current conduction across this sulfide device and that the current behaved in relation to the difference between the metals [12]. This was the first documented evidence of rectifying behavior with a current-voltage measurement. Many years later sulfide was replaced in patents and publications with several other more commonly known semiconductors such as silicon and silicon carbide. An interesting side note is that in order to ensure the metallization layer deposited on the semiconductors was planar, the researchers would polish the surface of a "platinized", or platinum-deposited layer, and reflect light off of it as a mirror to check the distortion of the image reflection [13]. In the early decades of the Twentieth century, the earliest metal-semiconductor diodes were fabricated. These diodes were mostly made out of tungsten "cat's whisker" contacts that made a "point-contact" to a crystal chuck of semiconducting material, often a sulfide although more pure materials were used as well. This is shown in Fig. 1.1. The final culmination of an actual usable device in electronics is the development of the "large area rectifying diode," with selenium being evaporated onto large metal substrates. These were the early converters of AC to DC for power applications [14].

The biggest proposal of using a metal-semiconductor contact comes when the patents of J. E. Lilienfeld including the first ever field-effect transistor (FET) in 1925 declared a "method and apparatus for controlling electric currents" although the transistor would not be realized as a device for another 21 years when William Shockley at Bell Labs in 1947 demonstrated the first bipolar transistor's operation, an accomplishment that won him the Nobel prize in physics in 1956 [15]. This achieve-

ment alongside the research being conducted into doping the silicon to achieve more conductive semiconductors, led the revolution for more sensitive rectifying diodes, transistors and power electronics.

Interestingly, in the 70 year era between Braun and Schockley, none of the 


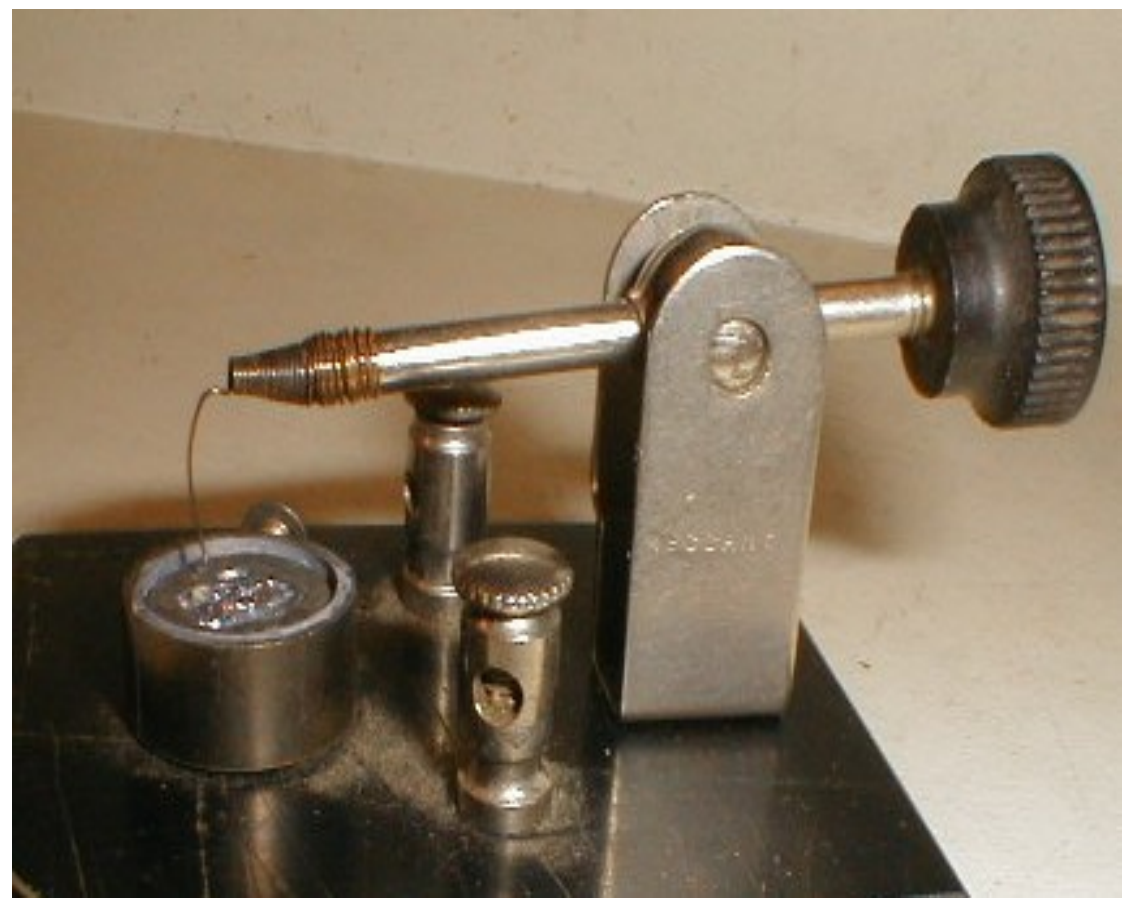

Figure 1.1: A "cat's whisker" device used in early radio broadcasting for creating point-contact rectifying diodes of a metal wire and semiconducting crystal to prevent current flow in a specific direction.

proposed theories of metal-semiconductor devices correctly predicted the direction of the rectification for a given metal-semiconductor diode. The first to attempt to derive a solution for this directionality was Nevill Mott in 1939. He reported that it is not the insulating layer of a semiconductor that causes the rectification but instead that when a metal and a semiconductor are in intimate contact, there is a barrier that forms which is proportional to the difference between the energy of the electrons in the lowest conduction band and the energy of the vacuum level, which we know to be the work function for metals, $\phi$, and electron affinity, $\chi$, for semiconductors, or more mathematically in Eqn. 1.1:

$$
\phi_{B}=\phi-\chi
$$

The use of the electron affinity, a property of the semiconductor, led Mott to develop a theory of conduction bands bending to form an equilibrium between the metal and semiconductor. This forms a continuous band from the potential barrier to the conduction band of the semiconductor. With this theory Mott assumed: 
- $\phi-\chi$ is larger than the fermi energy;

- Space charge due to currents in the blocking layer may be neglected;

- The mean free path is small compared to the depletion width, d;

- Nearly all of the potential drop of the barrier occurs in the semiconductor.

Mott was able to demonstrate that electron flow moves in the opposite direction of what was observed and that previous tunneling theories were incorrect in assuming the electron only travels through the barrier, but instead the electron must have enough thermal energy to pass through the barrier height [16].

Despite Mott's remarkable discovery, his predictions were flawed and disagreed with experiments. Mott's theory did not correctly account for donor/acceptor ions in the semiconducting layer; this was quickly added by Schottky-Spenke within a year $[17,18]$. This new Schottky theory took the constant electric field assumed in the semiconductor and showed that a linearly-decaying treatment agreed better for experimental data. Because of this change to the way the space charge was used, the barrier was named after Schottky as we know it today, the Schottky barrier. The final battle for the proper mechanism which caused this rectifying behavior was correctly proposed by Hans Bethe in 1942. In his paper, Bethe showed that the current was a thermionic emission of electrons over the potential barrier which indicated the kinetic energy of the electron must "go over" the barrier instead of tunneling through. Bethe's work brought the accepted theory from Mott's diffusion-drift mode to our current understanding of the thermionic mode of electron transport $[19,20]$.

\subsubsection{Ballistic Electron Emission Microscopy}

Shortly after the invention of the STM [21, 22, ?], two recent physics Ph.D. graduates, L. D. Bell and W. J. Kaiser, working for Jet Propulsion Laboratory in Pasadena, CA, developed a three terminal measurement utilizing the STM, which was able to tunnel electrons into a metal and then collect the ballistic electrons which made it through the metal and across the Schottky barrier at the interface of the metal and semiconductor, now known as ballistic electron emission microscopy [23]. This was the first technique able to directly image the subsurface interface properties 
with nanometer spatial resolution. The first measurements were done on Schottky barrier systems only but later work on semiconductor heterojunctions, oxides, and other interfaces were also investigated [11].

Ballistic electron emission microscopy is an STM based technique which injects electrons into the metal layer of a metal-semiconductor interface and collects the electrons that overcome the barrier height [11]. In BEEM the STM tip is positioned along the surface while a voltage is applied between the tip and the grounded metal surface, generating a tunneling current as shown in Fig. 1.2. If the energy of the electron is high enough and the electron has sufficient perpendicular momentum, it will pass from the metal to the semiconductor and be collected as BEEM current, $I_{B E E M}$,

$$
\frac{I_{B E E M}}{I_{\text {Tip }}} \propto\left(V_{\text {Tip }}-\phi_{B}\right)^{n} .
$$

Bell and Kaiser's seminal work assumed a simple theory to understand the current flow through the diode as shown in 1.2 where $I_{B E E M}$ is the BEEM current, $I_{\text {Tip }}$ is the tip current, $\phi_{B}$ is the Schottky barrier, $V_{\text {Tip }}$ is the tip bias, with $n=2$ as a fitting exponent $[23,11]$. This model (BK Model) was without consideration of quantum tunneling effects or reverse bias conditions. An $n=5 / 2$ fitting parameter was later published with these considerations and is discussed in Chapter 2 [24].

Bell and Kaiser developed the BEEM technique in most of their published work, verifying the validity of current-voltage spectra versus BEEM spectra and qualifying BEEM imaging. In their original work, $\mathrm{Au} / \mathrm{Si}$ and $\mathrm{Au} / \mathrm{GaAs}$ heterostructures were investigated. These two systems were chosen because of their differences in reported Schottky barrier values. The $\mathrm{Au} / \mathrm{GaAs}$ barrier height is dependent on the defects that are present at the interface, alloying between the metal and semiconductor layers and interdiffusion. The opposite is true of $\mathrm{Au} / \mathrm{Si}$ Schottky barrier height, where data is reproducible and has very little variation [25, 26, 27]. This early worked proved that $I_{B}-V$ spectra taken with the STM using BEEM technique was in fact a direct measurement of the Schottky barrier height and the interface electrostatics of the Schottky barrer. An extension to this work resulted in the formalization of ballistic electron emission microscopy and ballistic hole emission microscopy (BHEM), where BHEM is the generation of electron-hole pairs in the metal base while reversing the 
bias between the STM tip and the metal base.

Many of the early published papers on BEEM spectroscopy attempt to clarify the differences between crystal structure, BEEM current, and thickness through the use of BEEM imaging, spectroscopy, and modeling of the current [28, 29, 30, 31, 32, 33]. Schowalter et. al. demonstrated the discrepancy between the conventional BK model and measured data of $\mathrm{Au}$ on $\mathrm{Si}(111)$ and $\mathrm{Si}(001)$, which showed identical BEEM current characteristics. They argued that the inelastic scattering by interface defects was not the main cause of similarity between the spectra. The best agreement with the data was found when elastic scattering in the metal base using a Monte-Carlo method showed the broadening of the momentum distribution of injected electrons. In this model, electrons with energy $\leq 1 \mathrm{eV}$ showed a shorter elastic mean free path than their inelastic length, which makes the author's argument for the role of elastic scattering being the dominant mechanism for the discrepancy more intuitive.

Prietsch gives further arguments for elastic scattering in the metal which are characterized by a probability of change in momentum [1]. Random elastic scattering during electron transport provide a parallel momentum change with these electrons being accepted into the available states of the semiconductor by an acceptance cone. This acceptance cone will be discussed in Chapter 2, but in general the cone's acceptance angles that cross the interface are determined by the energy of the electron and material parameters. Prietsch also states that the other case for the acceptance of the electron into the semiconductor would be dependent on whether or not the electron is selectively scattered into propagating states. This selective scattering occurs when the hot electrons have already made it into the interface region and are influenced by the band structure of the semiconductor. Silicide as well as metal-semiconductor states penetrating across the interface on the order of $1 \mathrm{~nm}$ would be examples of when selective scattering may occur.

Inelastic scattering in the metal film causes an attenuation of the BEEM current and is attenuated based on an energy-dependent mean-free path of the electron. These events are primarily driven by electron-electron scattering because of the low energy nature of BEEM measurements. Electron-electron scattering is dominant in the range of $10 \mathrm{eV}$ above the Fermi energy of the metal and the excitation of plasmons is a highly energetic, energy-dependent scattering that will not be present in BEEM 
measurements, as stated by Crowell and Sze [34]. The inelastic scattering of BEEM with a ballistic electron at some energy, E, above the Fermi energy, $E_{f}$, will result in an absolute energy loss to the system with a value between zero and the total energy of the electron, E.

Another phenomena that can affect the BEEM spectra is that the overall transmission collected as BEEM current can be reduced by impact ionization occuring in the semiconductor after the electron has made it across the barrier height. This can occur at higher tip bias when the electron's energy is above the band gap of the semiconductor. Inelastic scattering causes a secondary electron to be generated with a hole. These effects are showing in the BEEM schematic in Fig. 1.2.

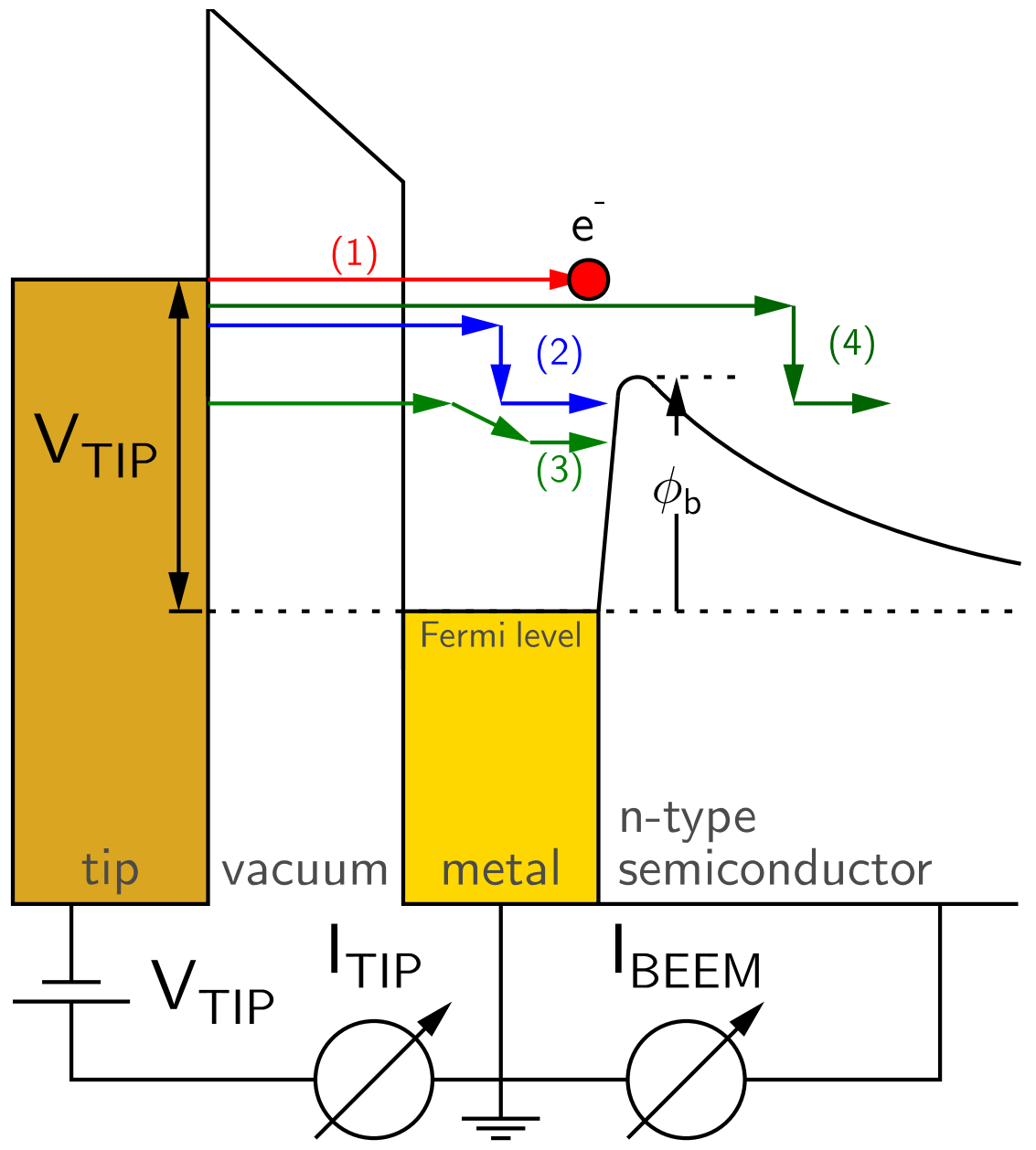

Figure 1.2: Diagram of BEEM measurement schematic. Electrons can have 4 different paths to overcoming the barrier: (1) ballistic transport, (2) inelastic scattering in the metal, (3) elastic scattering from grain boundaries, interface reflection, or selective scattering at the interface, (4) inelastic impact ionization 
Another major improvement in measuring the Schottky barrier after BEEM was introduced was achieved by comparing the sensitivity of BEEM current and barrier height when different sample preparation is involved. The benefits of BEEM are the high energy resolution on the order of $10 \mathrm{meV}$ and nanoscale probing of the interface. These two properties allow BEEM to investigate at interesting inhomogeneities and exotic metal/Si alloys to discern underlying Schottky barrier properties. One such study is on electron transport across the interface of high temperature annealing of $\mathrm{Au} / \mathrm{Si}(111)$ [35]. In Sumiya et al. gold was deposited on silicon (111) and BEEM spectra were taken after various annealing temperatures. Au dots with $3 \mathrm{~nm}$ thickness and $1.5 \mathrm{~mm}$ diameter were deposited on in-situ annealed $\mathrm{Si}(111)$ with a clean $7 \times 7$ surface confirmed by reflection high energy electron diffraction (RHEED) in a molecular beam epitaxy (MBE) system. Three samples were created with depositions performed at elevated substrate temperatures $300 \mathrm{~K}, 400 \mathrm{~K}$ and $573 \mathrm{~K}$. The highest temperature anneal was chosen to be below the eutectic temperature of $\mathrm{Au}-\mathrm{Si}$ at 636 K. Performing BEEM measurements on these samples revealed that the annealed samples showed $\mathrm{Au}-\mathrm{Si}$ interdiffusion which resulted in a disordered $\mathrm{Au}-\mathrm{Si}$ alloy at the interface. BEEM spectra were remarkably different, with the annealed samples showing a $500 \%$ decrease in the measured BEEM current at $2.0 \mathrm{eV}$ compared to the un-annealed. These results were recreated by annealing the room temperature sample at $573 \mathrm{~K}$ in-situ resulting in the same decrease in BEEM current beaing measured. The results concluded that a $\mathrm{Au}-\mathrm{Si}$ alloy with interdiffusion causes a large increase in scattering of the ballistic electrons which reduces the overall BEEM current and that care should be taken in measuring the substrate temperature before metal deposition.

A preference of using Au on Si to qualify new techniques and measurements of the Schottky barrier is noticeable by doing a literature review of BEEM. The benefit of $\mathrm{Au} / \mathrm{Si}$ measurements with BEEM is the inertness of $\mathrm{Au}$ when deposited on $\mathrm{Si}$ and the low scattering encountered in $\mathrm{Au}$ by the electron. This leads to relatively straightforward comparisons between measurements and between research groups using BEEM as a technique for measuring the Schottky barrier height. Similar use of the $\mathrm{Au} / \mathrm{Si}$ barrier height is used as a standard for data characterization in this work.

One of the first studies to employ measurement of the distribution of barrier heights across a $60 \times 60 \mathrm{~nm}$ area with $512 \times 512$ pixels was Palm et al. [36, 37]. The 
study used thin $\mathrm{Au}$ films on $\mathrm{Si}$ (111) to demonstrate a pixel-by-pixel approach to measuring the barrier height. $7.9 \mathrm{~nm}$ of Au was deposited by evaporating Au onto an hydroflouric acid (HF) cleaned Si surface. The authors used two seperate doping densities of Si, $N_{d}=1.5 \times 10^{14} \mathrm{~cm}^{-3}$ and $N_{d}=8.0 \times 10^{16} \mathrm{~cm}^{-3}$ to demonstrate fluctuations in the measured Schottky barrier between highly doped (HFLD) and low doped (LFLD) silicon. Measurements by BEEM with a pixel-by-pixel approach showed that the barrier height across the whole image varied by a guassian-like distribution of barrier heights that all had a goodness-of-fit $\left(R^{2}\right)$ better than 0.95 . The measured barrier height for HFLD was $0.801 \mathrm{eV}$ and for LFLD was $0.825 \mathrm{eV}$ with the distribution of barrier heights differing by up to $95 \mathrm{meV}$ at the extremities of the distributions but the weighted average differed by only $24 \mathrm{meV}$. The HFLD sample showed significant broadening of the histogram with the full width half maximum of $95 \mathrm{meV}$ compared to LFLD of $55 \mathrm{meV}$. Authors took effort to note the difference between the values of BEEM measurements versus more widely-accepted methods of reporting the Schottky barrier by current-voltage (IV), capacitance-voltage (CV), and photoelectric $(\mathrm{PE})$ measurements. The BEEM measurements agreed with $\mathrm{PE}$ measurements while the CV and IV measurements either over-estimated or underestimated the barrier height respectively. The study concluded that a more robust method of reporting the Schottky barrier is to develop a weighted average of barrier heights from the distributions.

Investigations into the distribution of barrier heights by several researchers [38, $39,40,41]$ shows that each distribution of barrier heights measured, account for fluctuations in electrostatics at the MS interface. Olbrich et al. were able to show that Co islands forming on GaAs surrounded by $\mathrm{Au}$ had two clear distinct distribution peaks at $1035 \mathrm{meV}$ and $1160 \mathrm{meV}$ respectively. As the thickness of Co increased from $0 \mathrm{~nm}$ Co to island growth and finally to a continuous Co layer, the peak of the distribution shifts from a single $\mathrm{Au} / \mathrm{GaAs}$ peak to many transition states showing a two peak distribution depending on the thickness. This demonstrated that the interface electrostatics is better represented by showing the distribution of the measured spectra.

With the capability of the STM, each of these unique spectra in the distribution are also able to be mapped out to their tip positions. Much like early BEEM imaging 
done on the interface, mapping the spectra to their STM tip positions presents a unique visualization of the interface coupled with a distribution of barrier heights. This visualization of the electrostatic barrier to nanoscale dimensions is a relatively recent BEEM advancement and is accomplished by mapping the barrier height using BEES [42, 43, 41, 44, 45]. Tens of thousands of spectra are collected on a regularly spaced grid and then individually fit to acquire a map of the local barrier height. The histograms of spectra thresholds contain information about the elastic scattering of the hot electrons and the interface stoichiometry [41, 44, 45]. This previous work has shown that growth of tungsten-silicide at the metal semiconductor interface increases the energy required to pass through the Schottky barrier by approximately $70 \mathrm{meV}$ over the similar tungsten-silicon bonded interface. This milli-electronvolt energetic resolution needed to resolve the barrier height can be measured with ballistic electron emission microscopy (BEEM) and modelled with an energy conservation kinetic Monte-Carlo method. The model is developed with the idea that a percentage of the electrons encountering the interface will be elastically scattered in the metal. These elastic scattering events can change the direction of the electron's momentum from perpendicular to parallel with respect to the interface. However, this early modeling neglected inelastic hot-electron scattering and scattering from ionized impurities in the near interface region. In addition, $\chi^{2}$ statistics were not utilized to find the best fit by performing exhaustive searches over the possible parameter space. A more robust model is needed to explain the electrostatics at the interface further.

\subsection{Thesis Overview}

This thesis aims to develop a computational model of the hot electron transport phenomenon and applied to many Schottky barrier height distributions measured with ballistic electron emission microscopy on a variety of metal-semiconductor systems. Thousands of BEEM spectra are acquired on various metal Schottky diodes over a square micron and used to generate a map and histograms of the thresholds. Monte-Carlo threshold modelling is utilized to illuminate the effects of localized barrier height formation and thickness-dependent transport. As well as, detection of multiple bonding species of barrier heights at the interface between a metal and a semiconductor through this modeling and measuring of the electrostatic barrier to 
nanoscale dimensions. The model finds the best agreement from $\chi^{2}$ minimization by performing large searches over parameter spaces as specified by the user. It captures the number of barrier heights at the interface, the elastic and inelastic hot electron scattering in the metal, as well as the presence of isolated charges near the interface. This model when combined with Schottky barrier maps and histograms enables discovery of the complex relationship between structural and electrostatic uniformity of a buried material interface with nanoscale resolution and called Schottky barrier visualization. 


\section{CHAPTER 2 \\ Theory of BEEM and Related Phenomena}

This chapter will be a brief overview of the topics necessary to understand the fundamental physics used in the measurements performed in this thesis. The topics discussed here are pertinent to the discussion of ballistic electron emission microscopy (BEEM) and modelling. The physics behind tunneling using the scanning tunneling microscope (STM) will be discussed as well as BEEM theory. Further discussion of the Schottky barrier formation will be shown with emphasis on Schottky-Mott theory, pinch-off effect, and scattering in metals. Finally, modelling efforts will be shown and statistical representation of the data-model fitting is described as well.

\subsection{Scanning Tunneling Microscopy}

In October of 1986, the Swedish Academy of Sciences issued a press release honoring Ernst Ruska "for his fundamental work in electron optics, and for the design of the first electron microscopy" and honoring Dr. Gerd Binnig and Dr. Heinrich Rohrer of IBM research laboratory in Zurich, Switzerland "for their design of the scanning tunnelling microscope." Binnig and Rohrers' design of the STM was a breakthrough in understanding the structure of matter and revolutionized what was thought to be imaging of a surface without direct electron microscopy or light optics. Instead, the STM uses mechanical motion and current feedback to probe the structure of the surface with angstrom resolution and relies on the quantum mechanical tunneling by maintaining a gap distance away from the conducting surface $[22,46]$.

The tunnel gap is the main physical concept of the STM. An atomically sharp metal tip is brought within the range of a few angstroms from the surface. This distance is the start of the quintessential potential barrier problem in quantum mechanics and solving this problem will provide information about required voltages, parameters and materials for measuring with the STM. As with most quantum mechanical problems, the best approach is to start with the time-independent Schrodinger equation: 


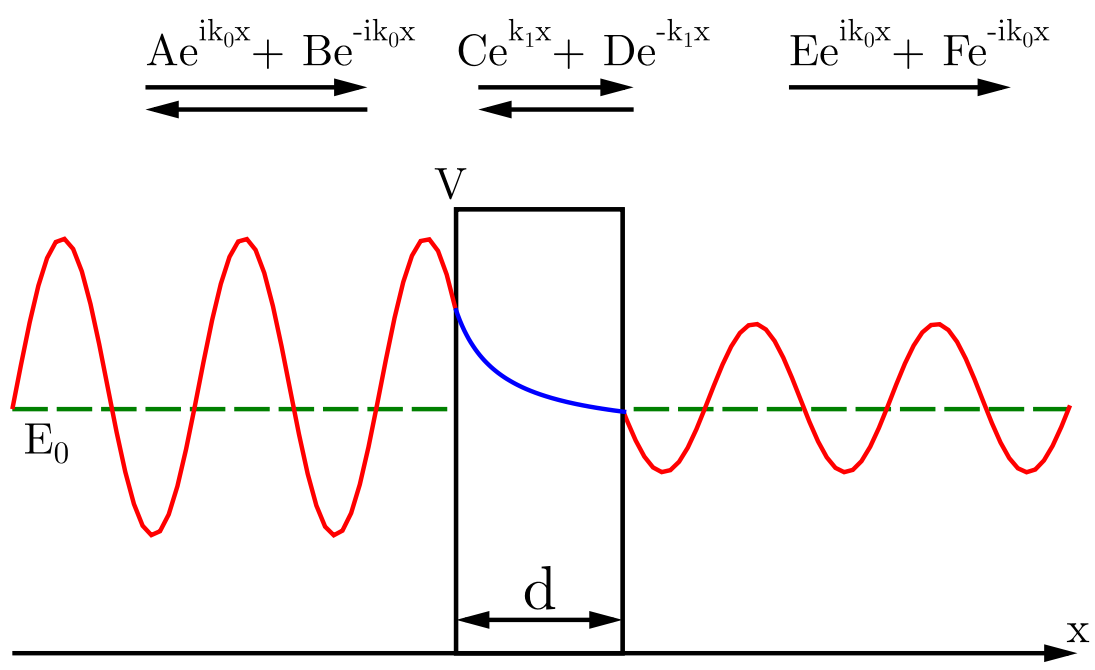

Figure 2.1: Tunnel bands for electron tunneling through a potential barrier.

$$
\left[-\frac{\hbar^{2}}{2 m} \frac{d^{2}}{d x^{2}}+V(x)\right] \psi(x)=E \psi(x),
$$

with $\psi(x)$ as the wave function, $E$ as the energy of the free particle and $V(x)$ as the perturbed potential. For the potential barrier problem there are two cases to be considered, $E>V$ and $E<V$. The second case is the tunnel barrier problem which is what will be focused on. The first thing is to solve for the wave equation for a free particle which results in

$$
\psi(x)=A e^{i k_{0} x}
$$

This is the solution to the wave equation, $k_{0}$ is the wave number for a free particle traveling in the positive $\mathrm{x}$ direction, as shown in Fig. 2.1. Other solutions can be found for a reflected particle traveling to the left in the negative $\mathrm{x}$ direction as well as a particle tunneling through a barrier with a given potential, $V$, with a width $d$. This leads us to a piecewise function of Eqns. 2.3, 2.4, and 2.5 with wave numbers indicated in Eqn. 2.6.

$$
\psi_{L}(x)=A e^{i k_{0} x}+B e^{-i k_{0} x} \quad x<0
$$




$$
\begin{gathered}
\psi_{C}(x)=C e^{k_{1} x}+D e^{-k_{1} x} \quad 0<x<d \quad(I I) \\
\psi_{R}(x)=E e^{i k_{0} x}+F e^{-i k_{0} x} \quad x>d \quad(I I I) \\
k_{0}=\frac{\sqrt{2 m E}}{\hbar}, k_{1}=\frac{\sqrt{2 m(V-E)}}{\hbar}
\end{gathered}
$$

These are the three equations which describe the particle traveling unbound and within a tunnel barrier in the three distinct regions shown in Fig. 2.1. Region I is the particle travelling from the left, region II is the potential barrier, and region III is the transmitted particle after tunneling. In region $\mathrm{I}$, the coefficients $A$ and $B$ are the amplitude coefficients of the travelling particle going left to right for $A$ and right to left after being reflected for $B$. $C$ and $D$ coefficients are the decay function while in the potential field. And finally the coefficients $E$ and $F$ are for the amplitude of the wave after the electron has exited the tunnel barrier potential and is traveling un-hindered by a potential field. Because of nothing hindering the particles' travel in region III, the coefficient for the wave traveling in the $-x$ direction, $F$, is zero and Eqn. 2.5 is now:

$$
\psi_{R}(x)=E e^{i k_{0} x} \quad x>d
$$

These coefficients can then be used to solve for the percentage of particles which are transmitted through the barrier and the percentage which are reflected by the barrier. The ratio of $B^{2} / A^{2}$ is the ratio of the number of electrons reflected at the tunnel barrier. Likewise, $E^{2} / A^{2}$ is the number of electrons which are transmitted through the barrier and are traveling in region III. This is also known as the tunneling current that can be measured in the STM as $I_{\text {tip }}$.

The STM can be used to provide a physical description of the tunnel barrier problem. Region I is the metallic STM tip, with the wave function of the periodic potential described as plane waves conducted through the atomically sharp tip. The tip is brought within a few angstroms' range of the conducting material and creates a tunnel gap which electrons are able to tunnel through with a voltage on the order of 


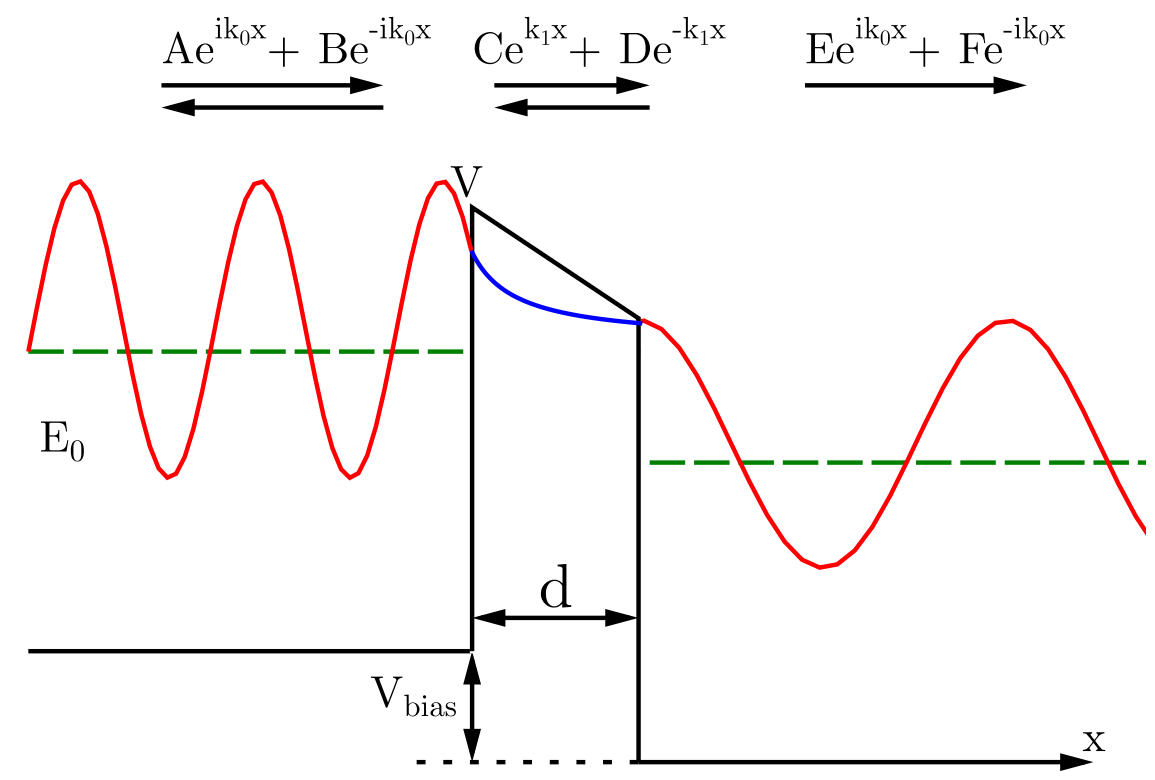

Figure 2.2: STM tunnel bands for electron tunneling through a potential barrier with an applied bias. Tunnel probability is controlled by Fowler-Nordheim tunneling.

$1 \mathrm{~V}$. Region II is the tunnel gap region and has the vacuum barrier with a potential energy that is, $4-5 \mathrm{eV}$ above the Fermi energy of the STM tip at $E_{0}$. The electrons can tunnel through this region when a bias is applied. Region III is the conducting sample which is providing energy states for the electron to tunnel into. For a plane wave to tunnel across the gap and be accepted in the conduction band of the conducting sample there must be available states which can accommodate a conducting electron. This is easily done with semiconductors and metals. The transmittance across this gap and into the accepted conducting film is provided by

$$
T(E)=|t|^{2}=\frac{1}{1+\frac{V_{0}^{2} \sinh ^{2}\left(k_{1} d\right)}{4 E(V-E)}} .
$$

When a bias is applied between the tip and the sample as in the setup of the STM, the tunnel barrier that the electrons need to overcome is no longer a rectangular barrier but instead a triangular barrier as shown in Fig. 2.2. In this special case the electrons that have to tunnel through this barrier now uses Fowler-Nordheim tunneling, or field emission [47]. Field electron emission refers to the emission of 
electrons when a large bias is applied between two conducting surfaces, in the case of the STM this is the STM tip facilitating the tunneling of electrons. To solve for this triangular barrier the Wentzel-Kramers-Brillouin (WKB) approximation must be used $[48,49]$. This approximation is done to assume that the wavefunction solution to the Schrodinger equation is in the form of plane waves and that any periodic potential or potential that slowly changes over spacial dimensions is able to be approximated by an exponential

$$
T \propto e^{-2 \int_{0}^{d} \frac{\sqrt{2 m(V-E x)}}{\hbar} d x} .
$$

It is assumed that $V=V(x)$ in the Fowler-Nordheim case and the tunneling probability is now dependent on the magnitude of the applied bias and the change in $x$ along the triangular tunnel barrier. This presents a unique case to have electrons tunnel from a STM tip with a specific given energy. The tunnel conductance is also dominated by the shape of the tip as well as the magnitude of the applied bias. Solving Eqn. 2.10 for a trapezoidal barrier as shown in Fig. 2.2 is

$$
T \propto e^{-V d},
$$

where $\mathrm{V}$ is the tip bias and $\mathrm{d}$ is the distance between the tip and sample.

\subsection{Schottky Barrier Formation}

The formation of the Schottky barrier at the interface of a metal and a semiconductor is a widely researched topic for the semiconductor industry and for solid state physics. For a general value and first approximation look at the value of the Schottky barrier height, the Schottky-Mott rule is able to predict a rough estimate based on the difference between the work function of the metal and the electron affinity of the semiconductor.

Schottky barrier formation is best described in three steps:

- The metal and semiconductor surfaces are brought into contact with each other.

- Charge flows from the semiconductor to the metal and a depletion width forms in the semiconductor. 


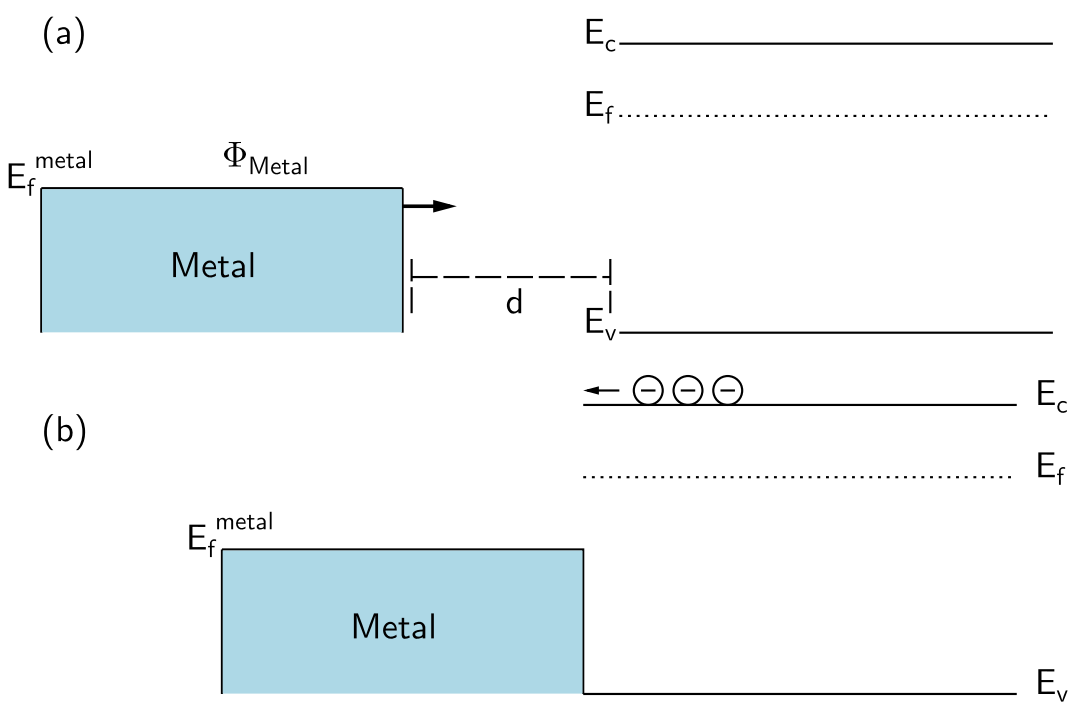

(c)

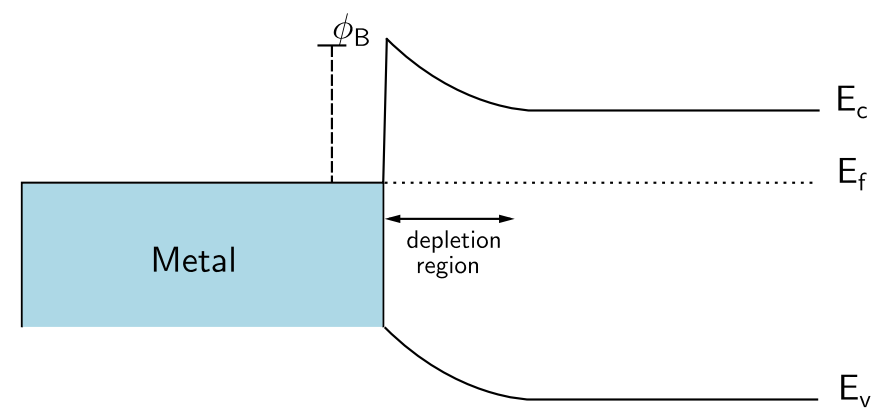

Figure 2.3: The formation of the Schottky barrier comes in 3 parts. (a) The metal and semiconductor surfaces are brought in contact with each other. (b) The electrons in the conduction band of the semiconductor flow towards the interface. (c) The conduction and valence bands bend in response to flow of charges to the interface and a Schottky barrier forms.

- The semiconductor bands bend in response to the depletion of charge and a potential barrier arises.

When the metal and the semiconductor surfaces come in contact, the large conductivity of the metal's "sea of electrons" and its Fermi level promote charges in the silicon to accumulate at the new interface. The semiconductor's Fermi level aligns itself with the Fermi level of the metal as indicated in Fig. 2.3. As charges migrate to the interface, a charge-depleted region of the semiconductor starts to form which is called the depletion layer. This depletion layer is dependent on the magnitude of the dopant density in the semiconductor. Also at this time, the conduction and valence bands bend in response to the redistribution of charge. The direction of the bending is also dependant on the type of dopant present in the semiconductor. 
At the interface of metal/n-type semiconductor the Schottky barrier height $(\mathrm{SBH})$ is the difference between the metal Fermi level and and the conduction band minimum of the semiconductor and the SBH of a metal/p-type semiconductor interface is measured between the valence band maximum and the metal Fermi level. In the case of n-type semiconductors the majority charge carriers are electrons which carry a negative charge, with electrons flowing from the semiconductor to the metal interface. p-type semiconductors have the majority charge carriers act as positive charges called holes. These charges or "lack of electrons" will flow towards the interface with electrons moving away from the interface. The carrier type of the semiconductor will affect the shape of the band diagram with n-type indicated in Fig. 2.3 and p-type semiconductor would have an inverse effect and the bands would bend downward towards the valence band.

$$
\phi_{B}=\Phi_{M}-\chi_{S}
$$

The Schottky-Mott rule in Eq. 2.11 shows that the Schottky barrier height, $\phi_{B}$ is equal to the metal work function, $\Phi_{M}$, minus the electron affinity, $\chi_{S}$. Unfortunately, this is an incomplete description of the barrier height and measured data disagrees with the value from only the difference between the work function of the metal and electron affinity of the semiconductor as shown in Fig. 2.4. Since the formation of the Schottky barrier involved many different phenomena occurring at the same time, the theories for the exact mechanisms which results in a specific Schottky barrier value are quite complicated. Many theories predict barrier heights based on orientation and structure of the interface and how well aligned the two lattices are matched [50, $51,52,53,54]$. Studying single crystal growth at the interface showed the change in the barrier height with pristine interfaces but were not able to theoretically predict the value $[55, ?]$. However, there are several supporting theories which do explain portions of the formation of the Schottky barrier successfully.

Cowley and Sze described the discrepancy between the Schottky-Mott model and the experimental data by an interface state creating a dipole between the metal and the semiconductor [56]. Dipoles that are created only a few atomic layers into the silicon have very large electric fields which cause the Fermi level of the semiconductor to be pinned at the interface. This pinning effect depends on a concept called the 


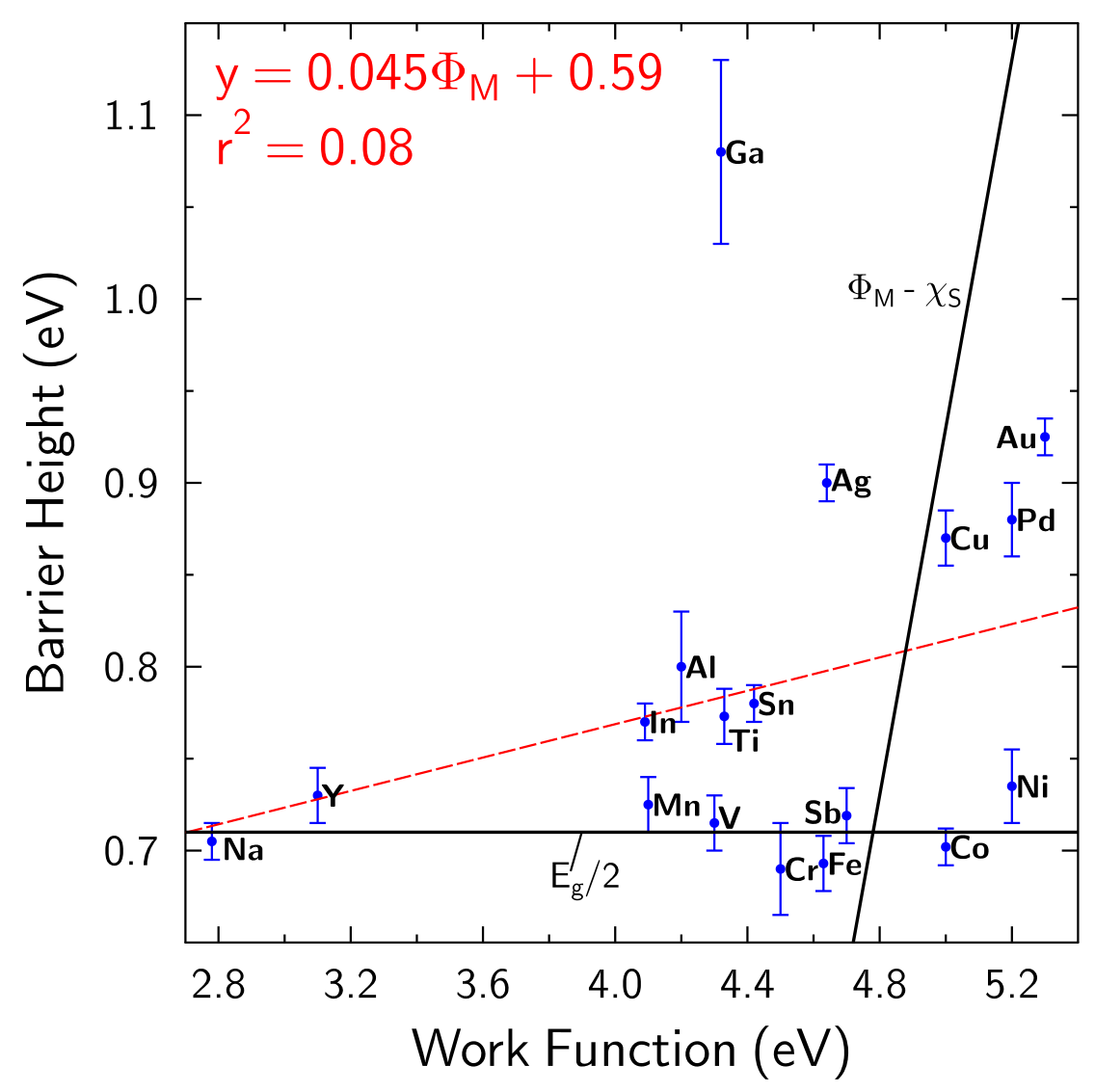

Figure 2.4: The Schottky-Mott relation does not follow reported measured data. Schottky-Mott relation is plotted for GaAs along with various metal Schottky barriers on GaAs. Linear fit to the data is also plotted to show disagreement with Schottky-Mott relation

charge neutrality level, CNL, and is described as the energy difference between the Fermi level and the level of filled charges at the interface. In other words, if the CNL is filled with negative charges, then the Fermi level is lower than the CNL and the first few atomic layers have a positive net charge. Inversely, if the CNL is filled with positive charges, the Fermi level is above the CNL and the surface is negatively charged. This leads to an energy level which the surface states must be filled for charge neutrality at the interface. The energy is the difference between the SchottkyMott relation and experimental data according to Eq. 2.12. Even further, the Fermi level pinning at the interface can be independent of the metal's work function and be a function of the doping and surface of the semiconductor or ignore surface effects and rely on the metal work function and electron affinity of the semiconductor with the CNL correction [57] 


$$
\phi_{B}=\Phi_{M}-\chi_{S}+e V_{i n t},
$$

where $V_{\text {int }}$ is the voltage of the interface dipole created.

One of the more popular recent theories introduces what are called MetalInduced Gap States, or MIGS. This theory relies on the fact that the wave function of a metal in close proximity to the semiconductor will tunnel into the semiconductor. This will induce electronic states in the band gap of the semiconductor. It was shown by Louie and Cohen that calculations of the electronic structure of the semiconductor close to the interface of a pseudo metal called "jellium" would result in a periodic potential change in the regions close to the metal but would decay more the further the calculation was from the interface $[58,59]$. The introduction of induced gap states is purely a function of the semiconductor but the periodic potential affects are metal dependant. The ionic properties of the semiconductor gives the decay length of the MIGS.

The MIGS theory has an issue with the magnitude of the the gap distance assumed between the metal and the decay of the MIGS. In the jellium model, it is shown that the typical decay length of MIGS is on the order of 0.1 to $0.3 \mathrm{~nm}$ for different semiconductors or around the distance of one lattice spacing of the semiconductor. This would put the induced gap states exactly at the interface but does not explain the location or dependence on the band bending outside of the interface. Tung showed that the contribution of MIGS is considered a more important part of the formation of the interface dipole states [58].

The barrier height of a silicide has been described by a more empirical model. Andrews et al. showed that the formation of the Schottky barrier in transition metalsilicide diodes is strongly correlated with their heats of formation, $\Delta H_{f}$, the measure of the energy released or consumed when one mole of a substance is created under standard conditions from its pure elements [60]. The heats of formation were normalized per metal atom per formula unit and a linear correlation was formed with heats of formation vs Schottky barrier heights. The analysis of why this correlation exists depend on the nature of the bonding for the transition metals. They state that the transition metals exhibit weak ionic structures with planes of strongly bonded atoms [61]. If the metalloid bonds are long and the interactions weak, increasing the 

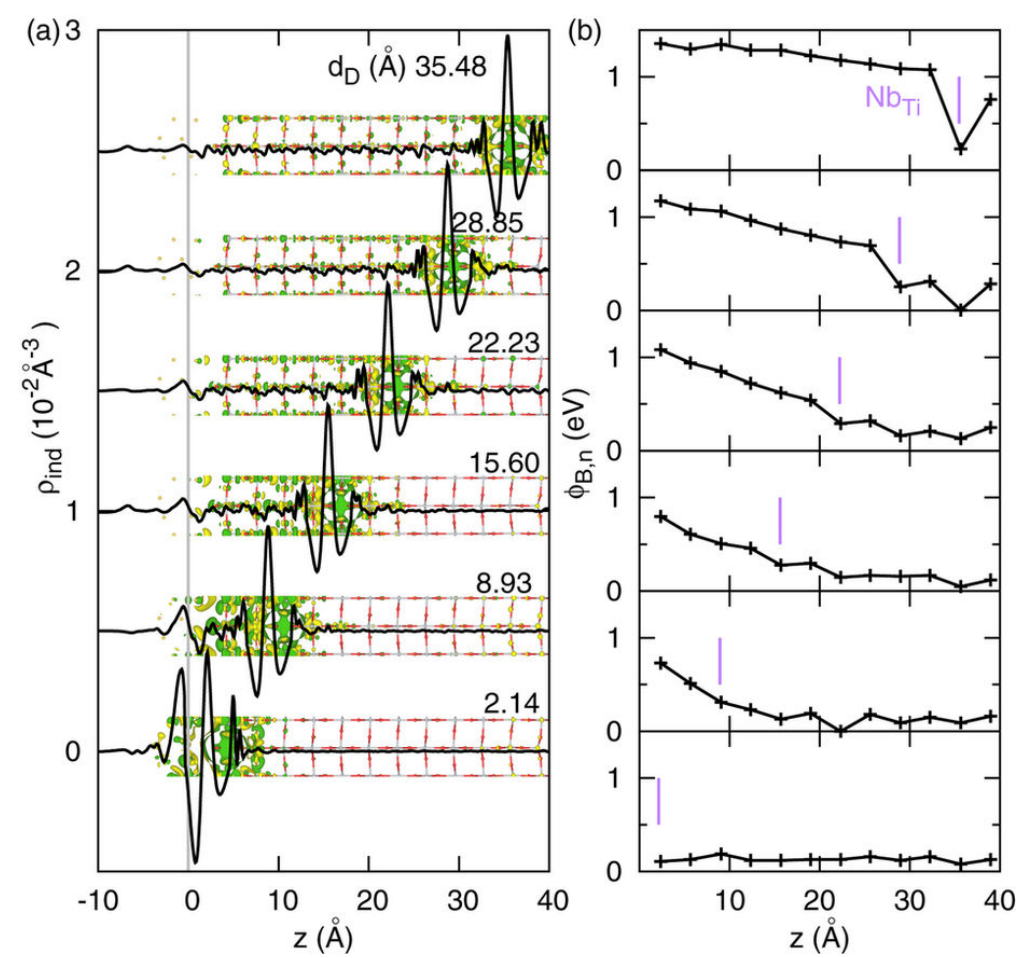

Figure 2.5: (a) The induced charge density from $\mathrm{Nb}$ dopants in the $\mathrm{TiO}_{2}$ lattice. (b) Band bending with the vertical bar indicating the depth location of the $\mathrm{Nb}$ dopant atom.

enthalpy of formation mixes more $s p^{3}$ orbitals with transition metal d-orbitals. With a strong mixture of $s p^{3}$ orbitals mixed with d-orbitals the metal atoms would become similar to Si and the barrier height approaches zero.

Recent explanations of barrier height formation are from density functional theory (DFT) calculations [62]. In this study, Jiao et al. looked at the potential barrier formation and band bending from an atomistic perspective with a deep level model (DL). The Schottky barrier investigated was $\mathrm{Nb} / \mathrm{TiO}_{2}$ and its value ranges from 0 to $1.26 \mathrm{eV}$ depending on the location of the dopant atom in the semiconductor. In Fig. 2.5, the location of the dopant atom in the $\mathrm{TiO}_{2}$ relative to the interface shows that the band bends linearly after a dopant atom is placed in the interface region. As the location approaches the surface, the barrier height shifts based on Eq. 2.13 with decreasing distance from the interface.

$$
\Delta \phi_{B, n}^{D L}=\frac{e^{2} a \sigma_{D L}\left(1-f_{D L}\right)}{\epsilon_{0} \epsilon_{S}},
$$


where

$$
f_{D L}=\left(1+\exp \left[\frac{\phi_{B, n}^{0}-e^{2} d_{D L} \sigma_{D L}\left(1-f_{D L}\right) /\left(\epsilon_{0} \epsilon_{S}\right)-E_{D L}}{k_{B} T}\right]\right)^{-1}
$$

and $d_{D L}$ is the distance into the interface and $\sigma_{D L}$ is the charge density in the interface. In their calculations, the characteristic width of the interface is $a=0.5 \mathrm{~nm}$ and point charge donors with energy $E_{D L}=0.2 \mathrm{eV}$ are below the conduction band minimum, $\epsilon_{S}=10, T=1000 \mathrm{~K}$. They concluded that the DL model is in very good agreement with experimental data when the model accounts for the barrier height reduction with dopant atom positioning near the interface.

\subsubsection{Image Force Lowering}

A phenomena that merits discussion with regard to measuring the Schottky barrier height is the lowering of the potential field at the interface due to an image force [4]. When a dopant atom in the semiconductor is within some distance, $\mathrm{z}$, from the interface of the metal and semiconductor, an image charge is assumed to be present in the metal with a magnitude equal to that of the carrier and opposite in sign of the charge and can be calculated using

$$
E_{\text {coloumb }}=\frac{-q^{2}}{8 \pi \epsilon_{s} z}
$$

where $\mathrm{q}$ is the charge, $\epsilon_{s}$ is the permittivity of the semiconductor, and $\mathrm{z}$ is the distance from the interface. This local change at the interface produces an electric field which interacts with the local interface dipoles which can affect the value of the barrier height. Image force lowering is dependent on the doping density of the semiconductor as more possible dopant atoms would be close to the interface and generate more localized electric fields. The change in the barrier height is calculated with Eq.2.16.

$$
\Delta \phi_{B} \approx \sqrt{\frac{2 * e^{3} N_{D} V_{b b}}{\epsilon_{s}}}
$$

In this work the impact of image force lowering is lessened by the use of low dopant density silicon for all experiments but this effect obviously has implications for manufacturing where very highly doped silicon is used often and for most devices. With 
an estimate of $8 \times 10^{3} \mathrm{~V} / \mathrm{cm}$ for depletion field of n-type silicon and an estimate of $3 \times 10^{3} \mathrm{~V} / \mathrm{cm}$ for p-type silicon, an approximate image force lowering is calculated to $\approx 2 \mathrm{meV}$

\subsection{Ballistic Electron Emission Microscopy}

During the deposition of the metal, defects appearing at the metal/semiconductor interface can affect the measured Schottky barrier height. The changes which affect the barrier height are typically studied with current-voltage and capacitance-voltage measurements, which measure thermionic emission of electrons across the Schottky barrier of the diode. Both methods require knowledge of the contact area and other assumptions and information about the semiconductor to calculate the barrier height. Ballistic electron emission microscopy is a technique which is able to probe the electrostatics of the interface directly without assumptions of material or varying methods of obtaining zero voltage current values. The technique also probes these electrostatics locally at a buried interface on the nanoscale enabling a look at interface defect and scattering sites that might affect the local barrier height.

\subsubsection{BEEM Spectra Modelling}

To model the ballistic electron transport from the STM tip to the collector base, the total current density across the metal/semiconductor interface is needed. The current density, $j_{c}$ is the total current that is collected after tunneling through a vacuum barrier, transport through the metal, including scattering, and across the potential step at the interface between the metal and semiconductor. These steps can be completed with a purely quantum mechanical approach by considering the potential functions for each of the stages. A thorough model was developed by M. Prietsch and R. Ludeke (PL) and is shown

$$
j_{c}=K \int_{E_{\min }}^{\infty} D\left(E_{x}\right) d E_{x} \int_{E_{0}}^{E_{\max }} f\left(E_{x}, E_{t}\right) T\left(E_{x}, E_{t}\right) F_{\theta}\left(E_{\max }, E_{t}\right) \Lambda\left(E_{x}, E_{t}\right) d E_{t}
$$

where $E_{x}$ and $E_{t}$ are the energy with momentum vector normal and transverse to the metal surface, respectively. The limits of integration are: $E_{\min }=E_{F}-e\left(V-\phi_{B}\right)$, 
$E_{\max }=r_{m}\left(E_{x}-E_{\min }\right)$ and $E_{\theta}=\left(e V+E_{x}\right) \sin ^{2} \theta D\left(E_{x}\right)$ is the transmission probability of overcoming a rectangular potential barrier, representing tunneling through the vacuum barrier as is shown in Eq. 2.8. $\Lambda\left(E_{x}, E_{t}\right)$ is the scattering probability of an electron in a metal and $f\left(E_{x}, E_{t}\right)$ is the Fermi function. $T\left(E_{x}, E_{t}\right)$ is the transmission across a one-dimensional step potential as has the generalized form of:

$$
T\left(E_{x}, E_{t}\right)=4\left[\frac{\left(m-m_{s}\right)\left(E_{\max }-E_{t}\right)}{m\left(e V+E_{x}\right)}\right]^{1 / 2} .
$$

The use of an average angle of incidence, $\theta$, is a new addition to this model which was not considered in early work by Bell and Kaiser (BK) [23, 11]. This incident angle is represented by a "co-ordinate transformation for off-normal angles of incidence" function: $F_{\theta}\left(E_{\max }, E_{t}\right)=\left(\left(E_{\max }-E_{t}\right) / E_{t}\right)^{1 / 2}$. Where $\theta$ is restricted to less than 20 degrees. $K=\left(\mathrm{em} / w \pi^{2} \hbar^{2}\right)$ is a set of constants with $w$ representing the depletion width in the semiconductor.

When $\theta$ is brought to 0 the transmission across the step potential is unity, and Eq. 2.17 is the same as it is in the BK model.

$$
j_{c}=K \int_{E_{\min }}^{\infty} D\left(E_{x}\right) d E_{x} \int_{E_{0}}^{E_{\max }} f\left(E_{x}, E_{t}\right) \Lambda\left(E_{x}, E_{t}\right) d E_{t}
$$

After integration the lowest order term can be discerned and gives a square law dependency of the voltage and Schottky barrier:

$$
j_{c} \propto\left(V-\phi_{B}\right)^{2}
$$

This is the equation given by what is considered the BK BEEM Model with $n=2$. This BEEM model is considered a good approximation for ignoring quantum mechanical effects at the interface as well as the electrons are considered normal incidence to the metal/semiconductor interface. When those effects are taken into consideration and the integral from Eq. 2.19 is calculated in full, a 5/2 power dependency is predicted by the lowest order. With the collector current:

$$
j_{c} \propto\left(V-\phi_{B}\right)^{5 / 2}
$$

The $5 / 2$ power is what is considered the PL BEEM model with $n=5 / 2$. These 
values of $n$ are used in the simplified BEEM model, Eq. 1.2, derived from expansion of terms from Eq. 2.19.

The difference between the two popular models shown here is determined by the source of the electrons and holes transmission across the Schottky barrier. In the case of BK model, the model predicts that the transmission across the metalsemiconductor interface is energy independent and is treated as a classical transmission probability across two materials, which shows that the change in applied bias does not alter the transmittance of the electrons across the interface. In the PL model, the authors explored an energy dependent transmission coefficient for the threshold values. Changing this transmission coefficient allows for quantum-mechanical transmission across a one dimensional potential step. In other words, the BK model is treated as a classical physics model and the PL model allows for energy-dependent quantum-mechanical tunneling to occur at the interface [63].

\subsubsection{BEEM Spectra Fitting}

Using the BEEM model with either choice of BK or PL power law is required to fit to the BEEM spectra. The percent transmission is typically utilized as the signal for the BEEM spectra. easier to understand. The percent transmission is calculated by dividing the BEEM current by the total current injected by the STM tip into the metal base as shown in Eq. 2.22.

$$
\frac{I_{B E E M}}{I_{T I P}}=A\left(V-\phi_{B}\right)^{n}
$$

Eq. 2.22 must be linearized into the form $y=m x+b$ by taking the $\mathrm{n}^{\text {th }}$-root of both sides. The slope, $m$, is equal to the nth-root of the amplitude of the BEEM spectra, $m=\sqrt[n]{A}$, and the y-intercept, $\mathrm{b}$, is proportional to the threshold of the spectra, $b=\sqrt[n]{A} \phi_{B}$.

In the fitting routine, the choice of a starting point is significant with a BEEM spectrum taking a shape similar to a current-voltage spectrum. This point in the routine is called the linearization start bias, LSB, and is usually the first data point in the spectrum. From the LSB, all data points thereafter have the nth-root calculated. A linear regression fit is started at a chosen bias called the fit start bias, or FSB. The FSB is the starting point of the fit window for the linear regression to be calculated. 


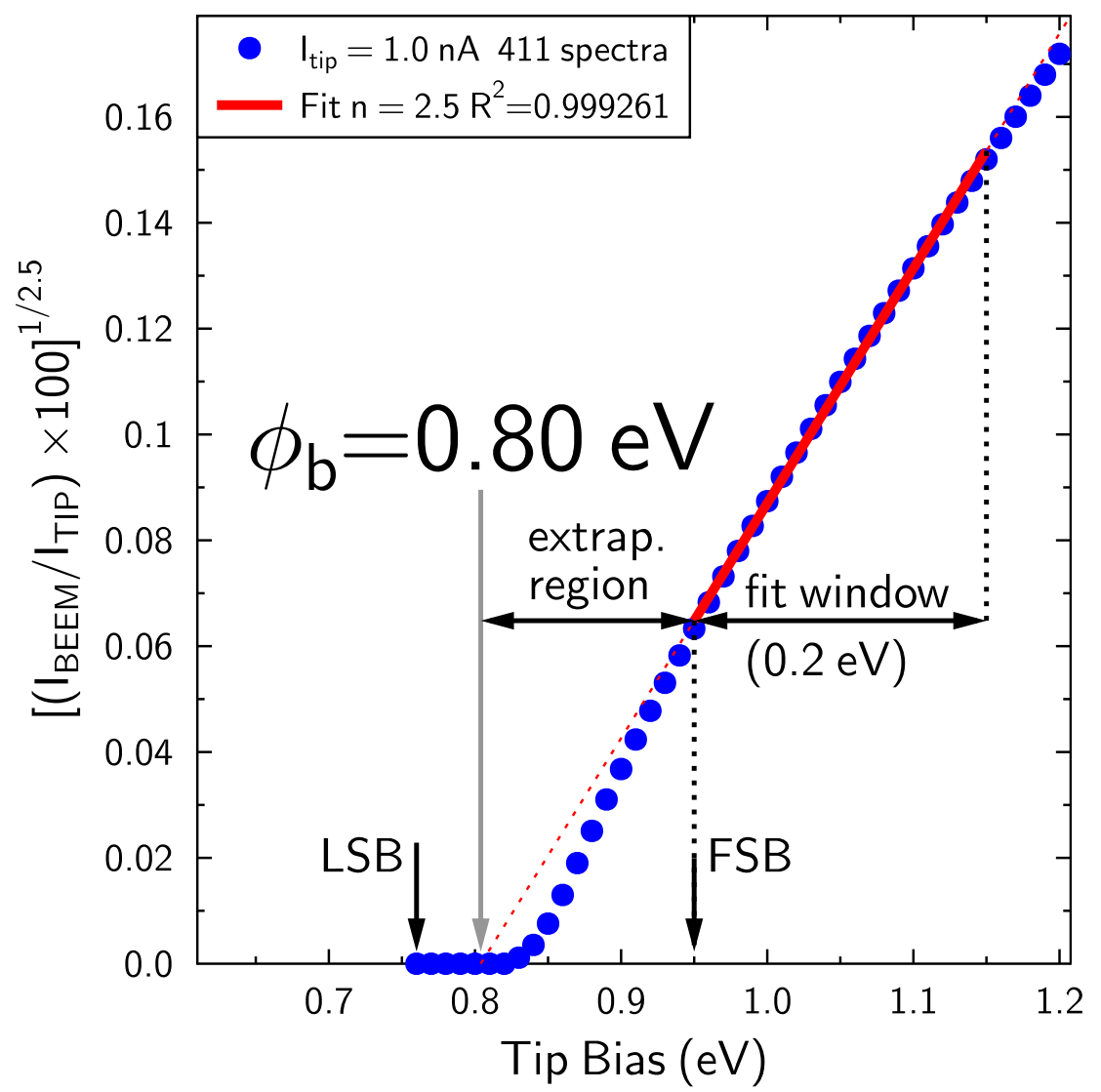

Figure 2.6: Typical BEEM spectrum for fitting routine. The LSB and FSB are chosen and maximizing the $R^{2}$ is done until a barrier height is found with the best fit to the data.

In general the fit window is on the order or $0.2 \mathrm{eV}$. Each point after the LSB is chosen as a new FSB and linear regression is again re-calculated and fit until the end of the spectrum is reached and all points have been fit. The second data point is then chosen as the LSB and the process is repeated until all points have been chosen as the LSB. These chosen starting points indicated in Fig. 2.6.

This routine is performed instead of using an initial guess of the parameters because not every spectrum will have the same shape or same characteristics. The simplicity of routine searching over all possible LSB and FSB values allows for finding the best fit to the data. The best fit to the data is chosen by the goodness-of-fit parameter, $R^{2}$, and fits typically fall within the range of 3 to $5 \sigma$ or 0.99 to 0.999999 . Once a good fit is found based on the maximization of $R^{2}$, then the linear fit is extrapolated to zero transmission and the barrier height is the $\mathrm{x}$-intercept for that spectrum. 


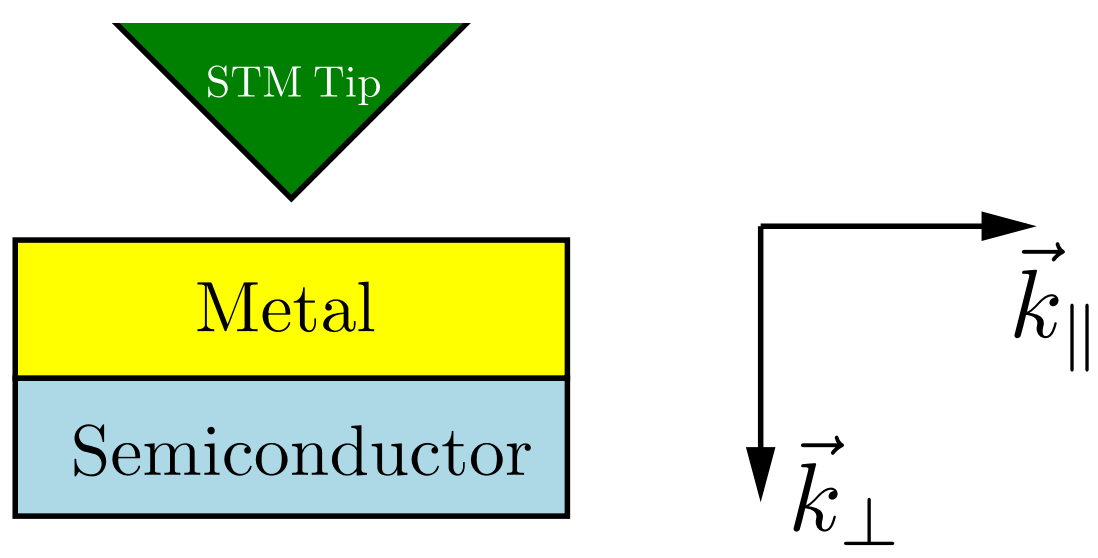

Figure 2.7: Total momentum representation as parallel and perpendicular momentum with respect to the interface of the metal and semiconductor.

\subsection{Electron Scattering}

One of the big insights gained in this work involves electron scattering in the metal base of the Schottky diode. There are many types scattering events which can occur but a broad look at the momentum of the electron will be discussed which includes: elastic scattering events and inelastic scattering events, specifically looking at the relative effect of the electron's momentum as it approaches the metal/semiconductor interface.

\subsubsection{Elastic Scattering}

Elastic scattering events are collision events which conserve energy of the particle when the event occurs, meaning that an electron with a given energy, E, and total momentum, $\vec{k}$, before an elastic scattering event will maintain the same energy and total momentum after the event. The direction of the momentum can change which can impact the BEEM current. For the sake of this work the total momentum will be split into perpendicular momentum and parallel momentum with respect to the interface of the metal and the semiconductor as shown in Fig. 2.7

The momentum $\overrightarrow{k_{T}}$ is the total momentum of the electron, $\overrightarrow{k_{\perp}}$ is the momentum vector that is orthogonal to the interface of the metal and semiconductor, and $\overrightarrow{k_{\|}}$ is the momentum vector that points in the direction parallel to the interface. The addition of the perpendicular and parallel momentums adds to the total momentum of the electron, 


$$
\overrightarrow{k_{T}}=\overrightarrow{k_{\perp}}+\overrightarrow{k_{\|}}
$$

where

$$
\overrightarrow{k_{\perp}}+\overrightarrow{k_{\|}}=m \vec{v}_{\perp}+m \vec{v}_{\|}
$$

The kinetic energy can also be defined in relation to the momentum

$$
K . E .=\frac{m v_{\perp}^{2}}{2}+\frac{m v_{\|}^{2}}{2}
$$

and this allows us to compare the perpendicular momentum to the barrier height

$$
\frac{k_{\perp}^{2}}{2 m} \geq \Phi_{B} \text { or } k_{\perp} \geq \sqrt{2 m \Phi_{B}}
$$

where eqn. 2.26 is a comparison between the kinetic energy associated with the perpendicular momentum and is the criteria needed for the electron to make it over the barrier height of metal-semiconductor system. This comparison is the crux of electron transport across the interface. In terms of momentum, if the electrons perpendicular momentum is large enough then the electron will be transmitted across the interface.

Typically, when discussing conservation of momentum of elastic scattering events careful consideration is taken to ensure that the electron's total momentum vector is what is conserved. In the case of BEEM measurements, whenever the electrons momentum is transferred from perpendicular to parallel, the electrons ability to surmount the barrier height is decreased. This can be shown with what is called an acceptance cone of energy in Fig. 2.8.

In this acceptance cone, Eqn. 2.27 is the critical angle subtended by the interface of the metal-semiconductor interface. The acceptance cone is specific to the energy of the electron, $E_{e}$, and the barrier height of metal-semiconductor system at that location of the STM tip. The Fermi energy, $E_{F}$, also gives more material-specific values of the critical angle

$$
\sin ^{2} \theta_{c}=\frac{m_{t}}{m} \frac{E_{e}-\Phi_{b}}{E_{F}+E_{e}}
$$

where the critical angle is the maximum angle which the electron can be de- 


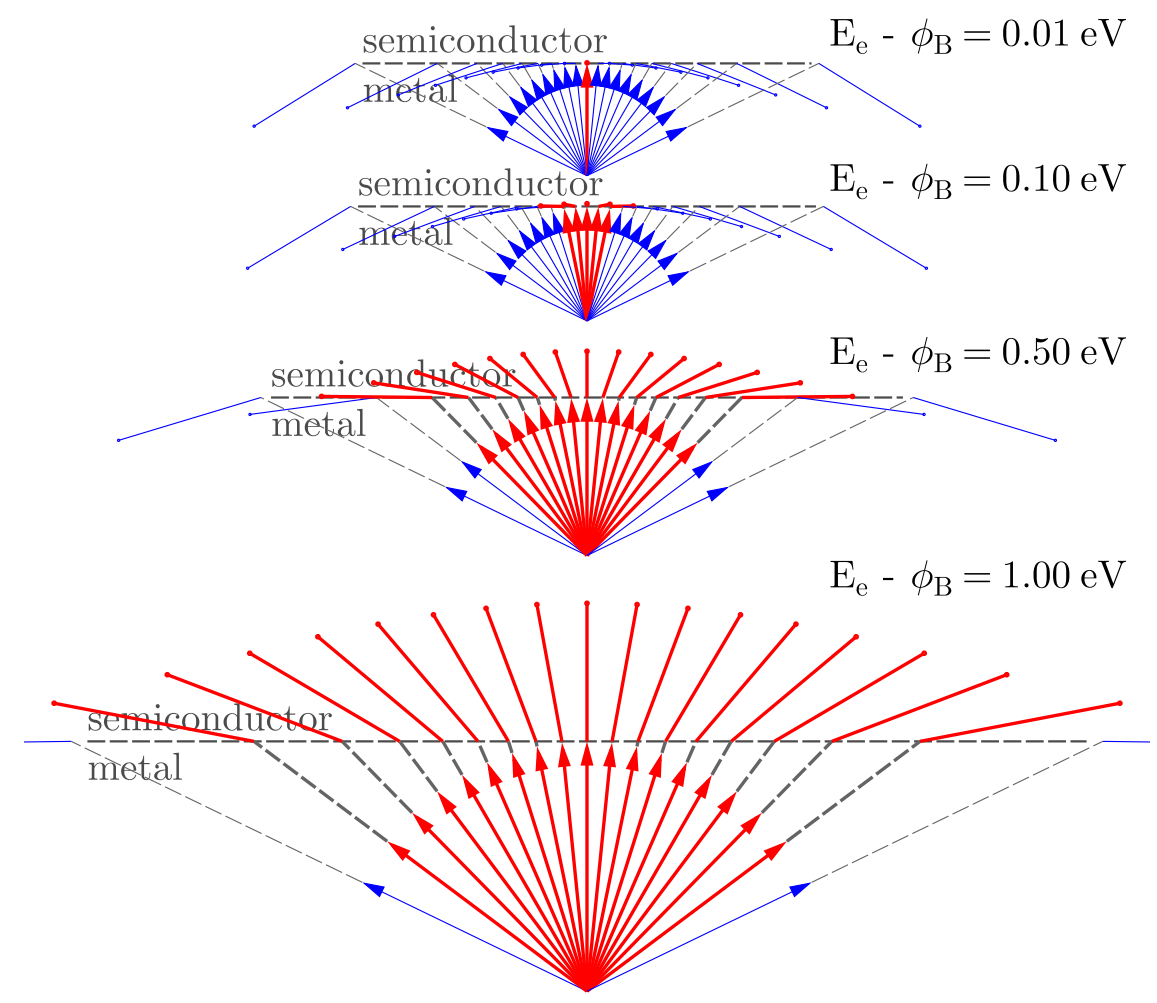

Figure 2.8: Acceptance cone of scattered angles as transmitted energy across the Schottky barrier with respect to the energy of the electron and the interface of the metal and semiconductor.

flected away from $\overrightarrow{k_{\perp}}$ due to elastic scattering. That is, if an electron is scattered at some angle away from normal to the interface, then the perpendicular momentum of the electron is decreased by some amount and the parallel momentum is increased by some amount. The total momentum of the electron is conserved but the perpendicular momentum of electron required to surmounting the barrier height has decreased.

Starting with an electron with $\vec{k}_{\perp}$ that is equal to the total momentum $\vec{k}_{T}$ and travelling in the direction of orthogonal to the interface:

$$
\mid \begin{aligned}
& \vec{k}_{\perp} \\
& \vec{k}_{\perp}=\vec{k}_{T},
\end{aligned}
$$


with

$$
\left|\vec{k}_{\perp}\right|=\sqrt{2 m V_{t i p}}
$$

If the electron travels without an elastic scattering event the kinetic energy of $\vec{k}_{\perp}$ is the same value as the tip bias from the STM and this value can be compared to the barrier height. If an elastic scattering event occurs the total momentum will remain the same, but the perpendicular momentum will no longer be equal to the total momentum. This will instead be equal to the sum of the parallel, $\vec{k}_{\|}$, and total momentum:

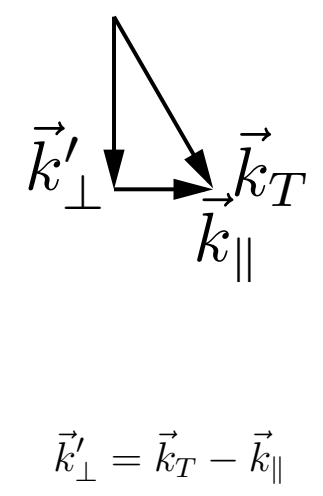

This new $\vec{k}_{\perp}^{\prime}$ is less than the original $\vec{k}_{\perp}$ and is thus less than the applied tip bias, $V_{t i p}$ :

$$
\vec{k}_{\perp}^{\prime}<\vec{k}_{\perp}
$$

Then the scattered perpendicular momentum can be compared to the barrier height

$$
\frac{k_{\perp}^{\prime 2}}{2 m} \geq \Phi_{B}
$$

This new "perpendicular" energy is no longer indicative of the total momentum of the electron from Eqn. 2.28. If the scattered electron is able to overcome the barrier height, this total energy is called the "threshold" since it is no longer simply describes a barrier height requiring a certain energy to overcome the barrier but also the increase of energy due to scattering. The decrease in the perpendicular momentum from elastic scattering, requiring higher energy to overcome the barrier, is what skews threshold histograms to higher energies with examples shown in the results section.

It is worth noting that none of the elastic scattering mentioned in this work 
cites a specific physical mechanism of elastic scattering. Several prominent scattering mechanisms which would generate elastic events include: electron-phonon, electrongrain boundary, and electron-defect interactions [64]. The effect of each interaction on elastic scattering is exacerbated by varying properties of the metal. The influence of grain boundary scattering might be hidden by surface roughness of the film or possibly the thickness of the film changes the orientation and size of the grains and a problem well discussed arises called the classical size effect and changes the resistivity of the film [65]. Also worth mentioning is the effects that are not limited to the physical aspect of scattering such as modifying the Debye temperature with a high surface/volume ratio could adversely affect the electron-phonon interactions. In general work done on the scattering in thin metal films is done by looking at the Mayadas-Shatzkes (MS) and Fuchs-Sondheimer (FS) models of scattering in metals which provides understanding of the elastic scattering in metals but this beyond the scope of this work $[66,67,68,69,70]$.

The general effect of electron scattering is the broadening of the ballistic electrons momentum distribution. This increases the mean angle of the ballistic electron emission spread and lowers the chance of acceptance into available states in the semiconductor when compared to the acceptance cone for the given energy and material.

\subsubsection{Inelastic Scattering}

As stated in Chapter One, the primary loss of ballistic electrons in BEEM measurements is elastic scattering, but inelastic scattering is still a significant fraction of the total scattering that an electron can encounter. Unlike elastic scattering, inelastic scattering is an energy dependent process and the total probability of the electron scattering inelastically is inversely proportional to the total energy of the electron squared, $1 / E^{2}$, and is captured by the inelastic mean free path

$$
\frac{1}{\lambda_{i}(E)}=\frac{A E^{2}}{\sqrt{\left(E+E_{F}\right)}},
$$

where $\mathrm{E}$ is the electron energy, $\mathrm{A}$ is a material specific scaling parameter of the metal layer, $E_{F}$ is the Fermi energy $[71,11]$.

Inelastic scattering events can lead to secondary electron generation in the metal 


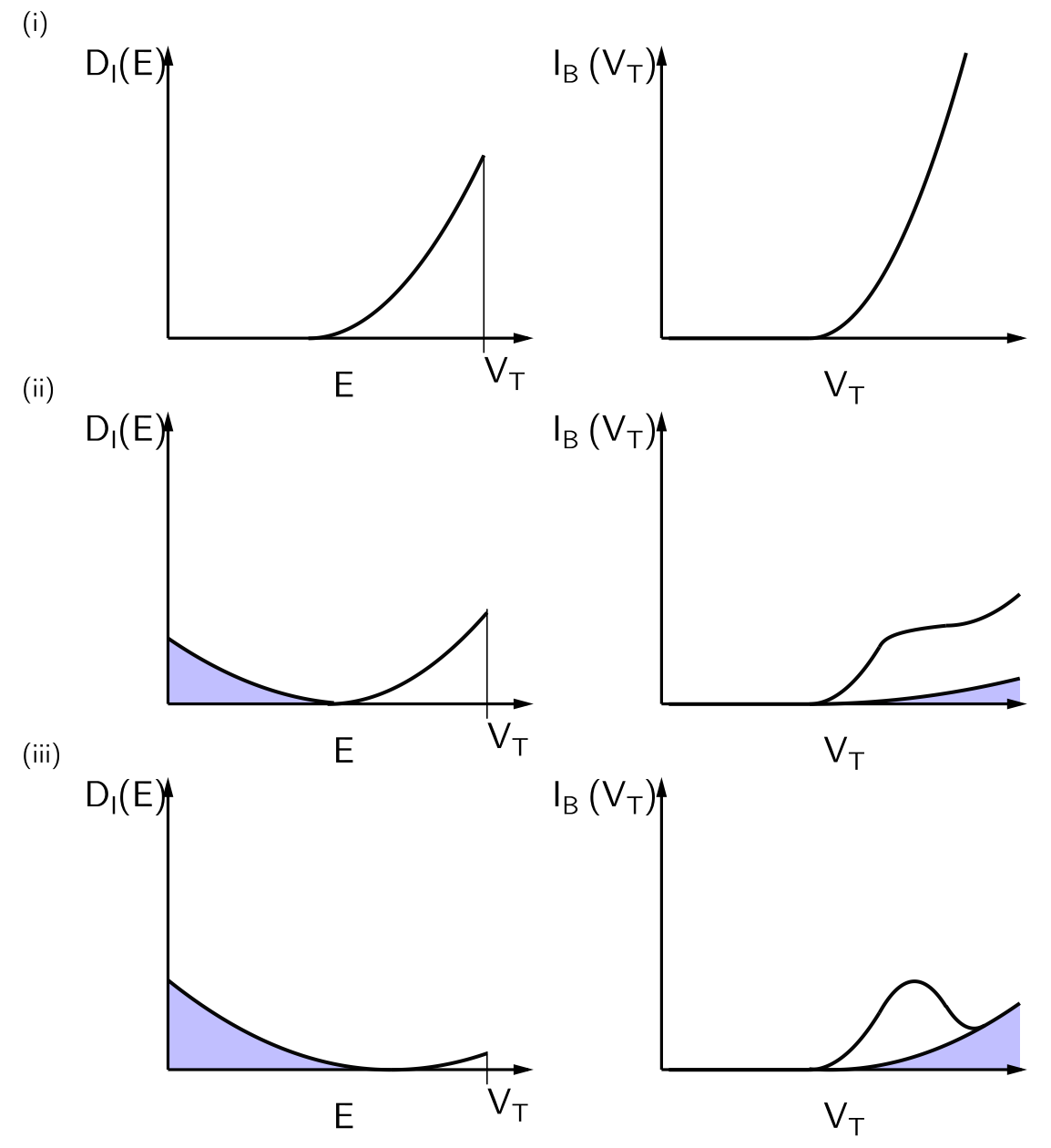

Figure 2.9: Examples of the energy distribution of electrons in the metal for large tip biases on the left column and the corresponding spectrum from a BEEM measurement on the right column. (i) The distribution of electrons at the interface for very thin metal film layers with all ballistic electron transport, (ii) electrons are inelastically scattered with thicker metal films and the start of secondary electron contributing to BEEM current, (iii) strong inelastic scattering that attenuates the signal and secondary electrons accounting for larger portion of BEEM current. Shaded blue region is contributions from secondary electrons [1].

which can also contribute to the BEEM current. The rate at which these secondary electrons are generated depends on the tip voltage and the energy of the inelasticallyscattered electron. Generation of the secondary electron will result in much lower energy electrons approaching the barrier interface. These electrons, when generated with energy greater than the Schottky barrier, can be collected as BEEM current. In general the contribution of these secondaries to BEEM current is shown in Fig. 2.9.

Each inelastic scattering event due to electron-electron interaction will have an 


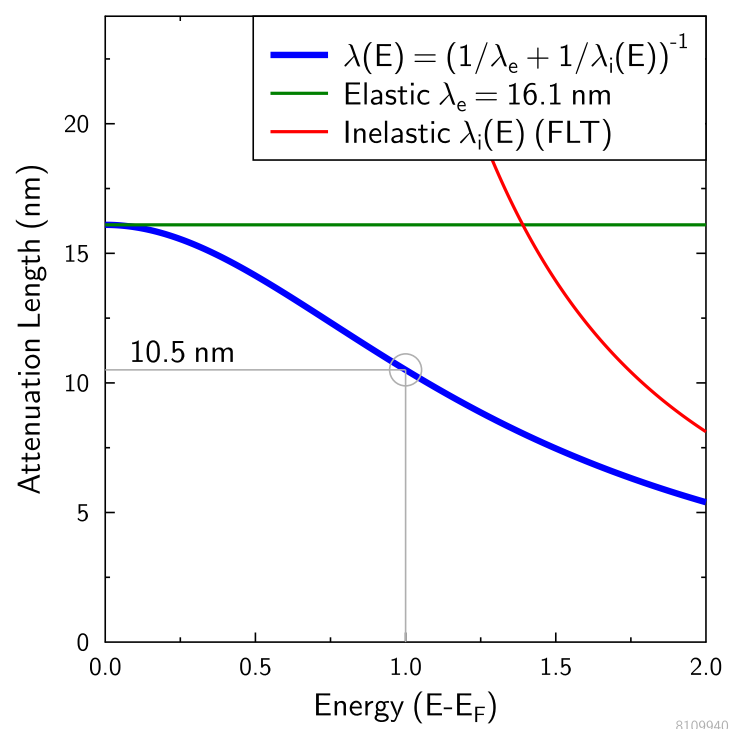

Figure 2.10: Scattering results based off of Fermi-liquid theory and the relation to the attenuation length. Example values are used to show comparison.

energy loss equal to $E / 2$ on average. This gives a rough estimate to the total energy loss to the system for a given electron that has made it through the interface and is collected as BEEM current. This is shown in the BEEM current schematic (ii) of Fig. 2.9, where at twice the threshold turn on there is a second threshold which starts collecting BEEM current from the inelastically-scattered electrons.

The scattering described in this chapter can be combined into a general scattering parameter using Mathhiessen's rule [72]

$$
\frac{1}{\lambda}=\frac{1}{\lambda_{e}}+\frac{1}{\lambda_{i}(E)},
$$

where $\lambda$ is the attenuation length, $\lambda_{e}$ is the elastic scattering length, and $\lambda_{i}(E)$ is the inelastic scattering length. The attenuation length is demonstrated in Fig. 2.10 with elastic scattering length as a constant value and the inelastic scattering length decreases in proportion to $1 / E^{2}$. The combination of these two values shows that at the Fermi energy the attenuation length is equal to the elastic scattering length and as energy is increased the attenuation length decreases towards the inelastic scattering length at higher energies. 

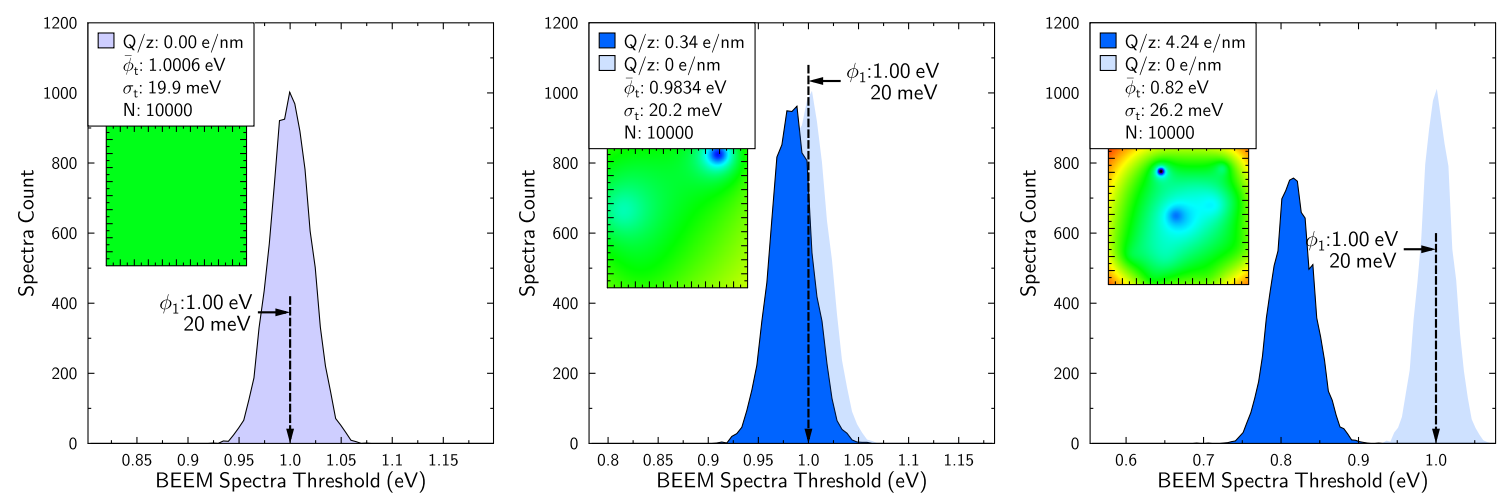

Figure 2.11: Computational modelling results and the effect of charge density. An increase in donor density decreases the barrier height. Units of charge are measured with depth from the interface in e/nm. Schottky maps are shown with each histogram to demonstrate visual representation of the barrier height changing.

\subsection{Modelling}

\subsubsection{Monte-Carlo Modelling}

Computational modelling of hot electrons and interface physics provides insight into the histograms of the BEEM spectra thresholds. The modelling computes histograms of voltage thresholds by simulating the transmission of the hot electrons through the metal and interface. The best fit between the modeled and measured histogram is determined by searching over billions of possible combinations of input parameters, while minimizing $\chi^{2}=\left(\sum_{i}^{N}\left(x_{i}-y_{i}\right)^{2} / y_{i}\right) /(N-D)$, where $x_{i}$ is the data, $y_{i}$ is the model, $N$ is the number of bins and $D$ is the number of degrees of freedom or parameters in the model. The search range and resolution for each input parameter is specified from either theory or empirical observation.

The physical effects in the model are broadly spilt into two categories; those arising from the interface, and those arising from the hot electron transport. The interface is modeled by specifying a Schottky barrier height $\phi_{b}$ along with a standard deviation $\sigma$. Multiple barrier heights can be specified with a mixture ratio $R$ to model the relative presence of each barrier at the interface. This captures any nonuniformities in the electrostatic potential that may arise due to structural or chemical disorder such as silicide formation or the presence of multiple metal species. Coulomb scattering from ionized impurity atoms in the near interface region of the semiconductor is captured by specifying the sign, magnitude, and number of the charges with a 

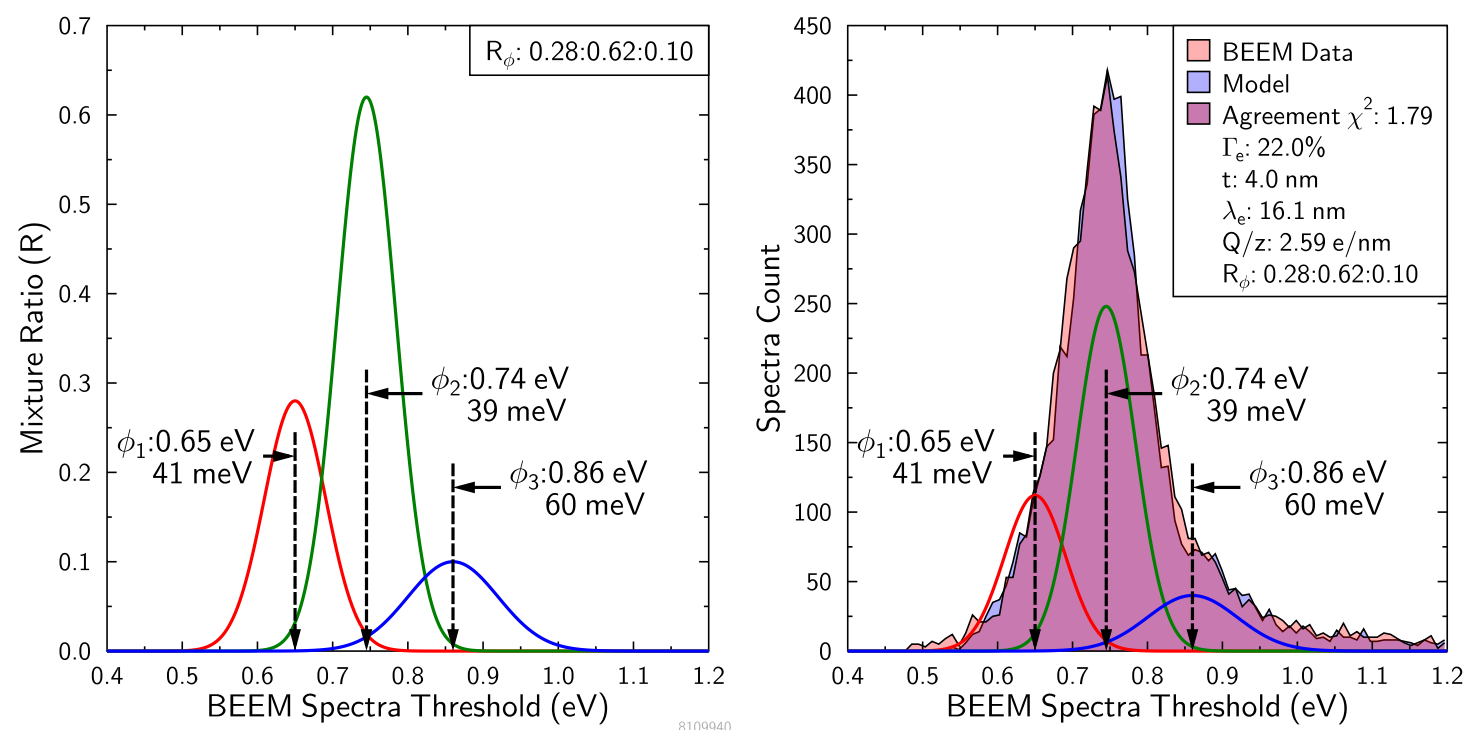

Figure 2.12: Computational modelling results and the effect of multiple barrier heights.

randomized position relative to the interface. The Coulombic potentials capture local adjustments to the electrostatic dipole potential from dopant atoms, or unintended charged foreign species introduced into the semiconductor during sample fabrication. Foreign species appear as thresholds above or below the mean barrier height for negative or positive charged impurities, respectively which is shown in Fig. 2.11. The temperature, binding energy, and dopant density are specified to model Debye length screening and ionization probability. The total charge per unit depth from the interface in units of $e / n m$ is recorded to capture this effect.

Specifying multiple barrier heights present at the interface also shapes the structure of the histogram as seen in Fig. 2.12. A Gaussian distribution of threshold values is assumed for each barrier listed before any scattering effects are added. A mixture ratio is chosen for the multiple barrier heights based on relative counts in the data at the energy values of each barrier height. This produces a summation-like distribution of threshold values as indicated in Fig. 2.12 on the left. Each barrier added to the modelling affects the overall values the model will find as a best fit. The best fit is purely a mathematical decision and analysis should be used to determine how many barriers and what values actually would be the most probable course. Data from BEEM measurements show the actual Schottky barrier height of the sample, and the skewing of the data collected is a combination of many different effects on the hot 

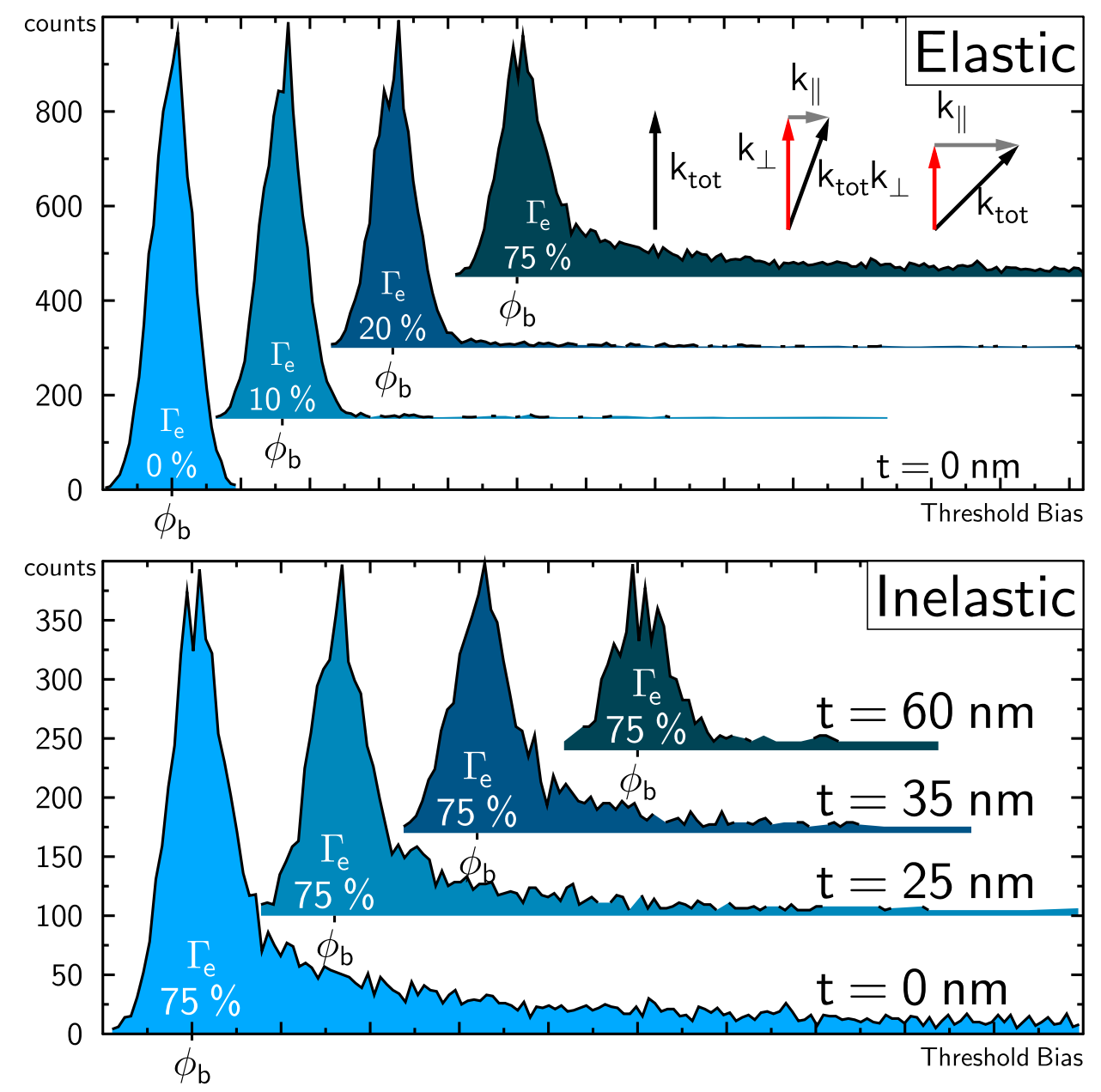

Figure 2.13: Computational modelling results and the effect of elastic and inelastic scattering. The elastic modelling shows a high energy tail appear while the inelastic modelling will have an overall large decrease in counts. $\Gamma_{e}$ is the elastic scattering probability and $t$ is an inelastic scattering length.

electron. The model aims to figure out the balance of values for all of these effects. The model is shown in Fig. 2.12 on the right and compared to the data. The bin size of both the data and the model are set to be equal to one another.

The hot electron transport and scattering phenomena in the metal are captured by a Kinetic Monte Carlo (KMC) approach using elastic and inelastic scattering parameters $\Gamma_{e}$, and $\Gamma_{i}$, respectively. The effects of elastic and inelastic scattering are shown in Fig. 2.13. $\Gamma_{e}$ specifies the fraction of spectra that experience an elastic scattering event, which randomly redirects the momentum vector away from the normal to the interface. This captures any momentum scattering that may arise from defects, grain boundaries, or other sources. When $\Gamma_{e}=0 \%$ the distributions are symmetric 
and become skewed to higher energies as $\Gamma_{e}$ increases as displayed in Fig. 2.13. $\Gamma_{i}$ is computed from the thickness of the metal in nanometers to compute the probability of an inelastic event occurring $\Gamma_{i}=1-\exp \left[-t / \lambda_{i}(E)\right]$, where $\lambda_{i}(E)$ is the inelastic mean free path and $E$ is the electron's energy. Fermi liquid theory (FLT) is utilized to model mean free path: $\lambda_{i}(E)=\sqrt{2 m_{e} E} /\left[a_{o}\left(E-E_{F}\right)^{2}\right]$, where $m_{e}$ is the electron mass, $E_{F}$ is the Fermi energy of the metal, and $a_{0}$ is a metal-dependant parameter obtained from theory (e.g.; $a_{o}^{A g} \approx 5.0 \times 10^{13} \mathrm{~s}^{-1} \mathrm{eV}^{-2}$ )[73] and is shown in

Fig. 2.10. Inelastic events reduce the total energy of the electron and greatly reduces the probability that electrons with high energies overcome the barrier and appear in the histogram as displayed in Fig. 2.13.

The model utilizes $\mathrm{C}++11$ coding and interfaces with a server storing all of the BEEM data collected for every sample measured. Random number generators are used to ensure that each data point in the model is random and systematic generation of numbers is reduced. The random number generators used are: mt19937, device_voltage, and taus8 from the BOOST $\mathrm{C}++$ libraries are utilized.

\subsection{2 $\chi^{2}$ Test}

Computational modelling and BEEM data are compared using a $\chi^{2}$ distribution. A $\chi^{2}$ distribution is primarily used for testing a hypothesis and understanding the confidence intervals in the critical region [74]. The purpose of looking at the $\chi^{2}$ distribution is to determine if the data and the model will either support or reject the null hypothesis. In the case of this model, the null hypothesis, $H_{0}$, is always stated as:

"Can it be proven, with a reasonable required level of significance, the null hypothesis that the modelled data set and the measured data set are created from the same population distribution function?"

Or by simply stating that the simulation expects to observe a particular set of parameters that will correspond to a lowered $\chi^{2}$ when compared with the measured data [75]. The $\chi^{2}$ tests require that an "alternate" hypothesis, $H_{a}$, is also put forth to have a second possibility in order to be able to reject the null hypothesis. In mathematical terms we state that the population density function, PDF, of the model is equal to the population density function of the measured data: 


$$
\begin{aligned}
& H_{0}: P D F_{\text {Model }}=P D F_{\text {Data }} \\
& H_{a}: P D F_{\text {Model }} \neq P D F_{\text {Data }}
\end{aligned}
$$

The $\chi^{2}$ test will be used to measure the difference between two population density data sets with binned data. For the test three variables are used for the calculation: $O_{i}$ is the observed variable used for the counts of the measured data, $E_{i}$ is the expected variable for the modelled binned counts and $\nu$ is the degrees of freedom as shown in Eqn. 2.37 and 2.38.

$$
\begin{aligned}
\chi^{2} & =\frac{1}{\nu} \cdot \sum_{i=1}^{n} \frac{\left(O_{\text {data }}-E_{\text {model }}\right)^{2}}{E_{\text {model }}} \\
\nu & =N_{\text {bins }}-1-N_{\text {parameters }}
\end{aligned}
$$

$\chi^{2}$ is normalized to the number of degrees of freedom for the given model data set. This is done in order to compare $\chi^{2}$ values across different models with different numbers of parameters. Since all $\chi^{2}$ values are normalized a minimization procedure can be developed to investigate all possible parameters for generating a population density of the model to match with the data. A significance level is also chosen to find the maximum and minimum possible accepted $\chi^{2}$ value for given degrees of freedom. If the $\chi^{2}$ value falls within the upper and lower limits of the values given by the significance level, the null hypothesis is fulfilled and our model parameters are in an acceptable range. If the values for $\chi^{2}$ are greater than the values from the significance level, then either the data is too perfect and fraudulent, the model is too low of a probability to be real, or our model parameters are chosen inaccurately and does not reflect agreement with the data, all would result in rejecting the null hypothesis. These significance values are found in Table 2.1 [75].

A rigorous approach to computational modeling involves asserting the level of confidence with each addition of parameters that changes the sum of the number of degrees of freedom associated with that model. In this work the calculation of the degrees of freedom, $N_{D}=N-D$, is used to normalize $\chi^{2}$ across all the models for comparison, but care must be taken to ensure that every additional parameter 


\begin{tabular}{c|ccccccccccc} 
DoF & \multicolumn{10}{|c}{$\chi^{2}$ value } \\
\hline 1 & 0.004 & 0.02 & 0.06 & 0.15 & 0.46 & 1.07 & 1.64 & 2.71 & 3.84 & 6.63 & 10.83 \\
2 & 0.1 & 0.21 & 0.45 & 0.71 & 1.39 & 2.41 & 3.22 & 4.6 & 5.99 & 9.21 & 13.82 \\
3 & 0.35 & 0.58 & 1.01 & 1.42 & 2.37 & 3.66 & 4.64 & 6.25 & 7.82 & 11.34 & 16.27 \\
4 & 0.71 & 1.06 & 1.65 & 2.2 & 3.36 & 4.88 & 5.99 & 7.78 & 9.49 & 13.28 & 18.47 \\
5 & 1.14 & 1.61 & 2.34 & 3 & 4.35 & 6.06 & 7.29 & 9.24 & 11.07 & 15.09 & 20.52 \\
6 & 1.63 & 2.2 & 3.07 & 3.83 & 5.35 & 7.23 & 8.56 & 10.64 & 12.59 & 16.81 & 22.46 \\
7 & 2.17 & 2.83 & 3.82 & 4.67 & 6.35 & 8.38 & 9.8 & 12.02 & 14.07 & 18.48 & 24.32 \\
8 & 2.73 & 3.49 & 4.59 & 5.53 & 7.34 & 9.52 & 11.03 & 13.36 & 15.51 & 20.09 & 26.12 \\
9 & 3.32 & 4.17 & 5.38 & 6.39 & 8.34 & 10.66 & 12.24 & 14.68 & 16.92 & 21.67 & 27.88 \\
10 & 3.94 & 4.87 & 6.18 & 7.27 & 9.34 & 11.78 & 13.44 & 15.99 & 18.31 & 23.21 & 29.59 \\
\hline P value & 0.95 & 0.9 & 0.8 & 0.7 & 0.5 & 0.3 & 0.2 & 0.1 & 0.05 & 0.01 & 0.001
\end{tabular}

Table 2.1: $\chi^{2}$ distribution table. DoF are the number of degrees of freedom assumed and $\mathrm{P}$ value is the probability associated with the $\chi^{2}$ value.

is meaningful. A level of confidence must be set to show that the two distributions agree without chance.

The P-values for the measured $\chi^{2}$ and $N_{D}$ of data and modeling presented in this paper are shown in Fig. 4.9 and computed utilizing

$$
\mathrm{P}-\text { value }=\int_{\chi^{2}}^{\inf } \frac{x^{N_{D} / 2-1} e^{-x / 2}}{2^{N_{D} / 2} \Gamma\left(N_{D} / 2\right)} d x
$$

where $\Gamma$ is the gamma function [75]. A $3 \sigma$ confidence level is chosen to determine valid fits to the data.

\subsubsection{Model Algorithm}

The model produces a histogram of barrier heights to compare to the BEEM data based on the physics of hot-electron transport and interface electrostatics. Parameters such as the Schottky barrier height, standard deviation, elastic and inelastic scattering rates, the number of desired barriers and position and depth of the charges at the interface are specified for each run of the model. A reproducible seed is generated from a time stamp for each simulation, which then is used to randomly populate a two-dimensional vector with a Gaussian distribution of barrier height values as specified. The size and shape of this Schottky barrier vector is made equivalent to the mapped grid of the interface that was used to acquire the BEEM spectra. Additional Schottky barrier heights are included in the vector if desired based on the ratio. If any 
charges are specified this vector is modified by calculating the net Coulomb potential from the aggregate set of charges for each position in the vector.

For each location in the Schottky barrier vector the following steps are performed to determine the measured barrier height at that location:

1. The elastic scattering angle is determined from a random number.

2. An inelastic scattering probability is determined from a random number.

3. For each energy (tip bias) in the measured spectra, the inelastic scattering probability is calculated and if it occurs the electron's energy is reduced by a specified amount. Then the elastic scattering angle is applied and the energy associated with the resulting forward momentum is compared to the Schottky barrier at that location. If the barrier height is less than this "forward" energy then the tip bias is added to the output histogram as the measured barrier height at that location.

4. A $\chi^{2}$ value is computed from the model and data histograms, always keeping the best fit. The simulations end when the entire parameter space has been simulated.

Two types of random number generators are used (taus88 and random_device) [76]. The former is a Tausworthe generator which uses a polynomial initialized by the time stamp generated from the reproducible seed. The latter is non-deterministic and uses implementation-defined stochastic processes to create sequences of random numbers that are uniformly distributed. 


\section{CHAPTER 3 EXPERIMENTAL METHODS}

This chapter will focus on the process, steps and aspects related to measurement of Schottky devices measured with BEEM. This includes all work completed before BEEM measurements such as metal deposition, sample preparation and mounting to custom STM plates, as well as the BEEM measurement setup and acquisition of data.

\subsection{Sample Preparation}

\subsubsection{Substrate preparation}

The first aspect of setting up a BEEM measurement involves the proper care and selection of the substrate prior to metal deposition. The substrate for all BEEM studies used in this work was single-crystal silicon with a $<001>$ orientation of the crystalline surface structure. The choice of this substrate comes down to convenience more than functionality. When $<100>$ is cleaved, the primary flat of a wafer will cleave at angles of 90 degrees to initial stress fracture. This produces cleaved wafers that are in a regular quadrilateral shape which in practice gives more control over the region for metal deposition. Another good reason is that it is the common orientation used in the semiconductor industry as well as a few other effects of choosing $<100>$ over $<111>$ which were discussed earlier [77, 78, 79]. The resistivity of the silicon substrate is chosen to make the most of a longer depletion layer in the silicon. Typically this is on the order of microns into the silicon. A $2 \mu \mathrm{m}$ depletion width in an n-type wafer is achieved with a resistivity of the silicon measured to be $100 \Omega$-cm which correlates to a doping density of $\approx 10^{13} \mathrm{~cm}^{-3}$. The same is done for p-type wafers with a doping density of around $\approx 10^{15} \mathrm{~cm}^{-3}$, resistivity of $10 \Omega$-cm and a depletion width of $0.5 \mu \mathrm{m}$.

The choice of the substrate also affects how the surface needs to be cleaned to achieve proper metallic contact with the semiconductor. Each substrate material (Si, Ge, GaN) behaves differently on the surface and how the semiconductor's oxide forms. For silicon, a thin oxide grows on the surface from dangling bonds of the top surface layer of silicon. These dangling bonds will bond with oxygen and form 
a $0.2 \mathrm{~nm}$ native $\mathrm{SiO}_{2}$ oxide layer on the surface after 20 minutes in air, and after 3 hours will form a $1 \mathrm{~nm}$ thick oxide layer which is the self-limiting point at standard room temperature and pressure [80]. This oxide growth is often described using the Deal-Grove model [81]. The reason that it is important to know about this thin oxide layer is that BEEM is very sensitive to growth on the surface. A simple thin native oxide can be detrimental to any metal-semiconductor measurements done with BEEM as it causes electron scattering and an increase in the momentum needed to traverse the interface. To remove the oxide, all substrates are immersed in a $49 \%$ hydroflouric acid ( $\mathrm{HF}$ ) bath for 5 seconds and rinsed with distilled $\mathrm{H}_{2} \mathrm{O}$. The acid bath etches the native oxide at a rate of $2300 \mathrm{~nm} / \mathrm{min}$ and leaves the surface of the silicon terminated with hydrogen which is illustrated in Fig. 3.1. The hydrogen-terminated surface is able to inhibit oxide growth for up to 20 minutes after etching in atmosphere [82, 83]. Immediately after etching the substrate is loaded into the metal deposition system and pumped down to UHV conditions, preventing oxide growth.

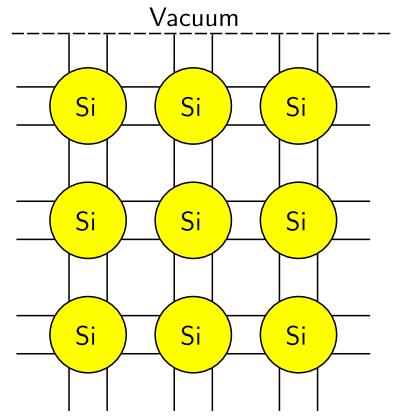

a) Silicon Surface

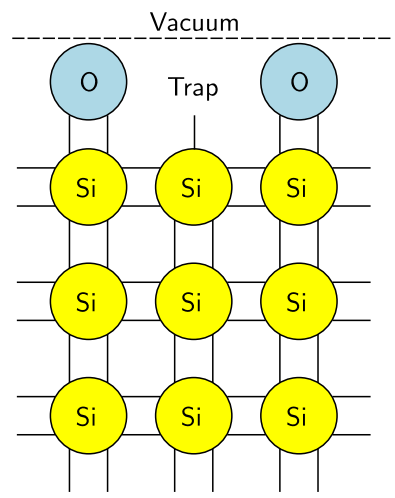

b) Unpassivated $\mathrm{Si} / \mathrm{SiO} 2$ interface

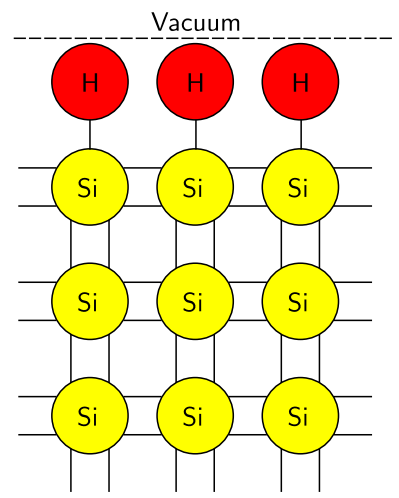

c) H-terminated surface

Figure 3.1: Diagram showing the silicon surface a) before exposure to oxygen, b) after $\mathrm{SiO} 2$ formation on the surface, and c) after hydrogen passivation of the surface with $\mathrm{HF}$ etch of the native oxide.

\subsubsection{Metal depositions}

Metal deposition is a critical step in the creation of a Schottky junction. The decision of which metal to use for a device is a widely changing part of the semiconductor industry ranging from aluminum as the first metals used as a contact to silicon or more complicated metallic structures such as Ti-Ni-Pt/Si. A wide array of metals 
are used in this thesis, with multiple metal deposition methods performed depending on the type of metal grown.

Electron beam evaporation was utilized to deposit a tungsten film with a gold capping layer with a base pressure of $10^{-8}$ Torr. E-beam evaporation is a physical vapor deposition method relies on an electron beam generated by a charged tungsten filament in vacuum which bombards a target source material increasing the temperature of the target eventually causing the material to enter a gaseous phase [84]. The fact that it is a thermal evaporation process is important for later analysis. The gold capping layer prevents oxidation of the barrier metal during sample mounting. All metal films were deposited with a $1 \mathrm{~mm} \times 2 \mathrm{~mm}$ shadow mask for a consistent dimensional area across all measurements performed.

The main metal deposition system used is a UHV molecular beam epitaxy (MBE) system with a separate chamber for radio frequency magnetron sputtering. Tungsten and chromium were sputtered onto a silicon (001) substrate using the magnetron sputtering chamber constructed in-house. The two deposition methods are connected in-situ, and both are used for each sample fabricated. Once the tungsten or chromium sputtering takes place, the sample is moved in vacuum to the $\mathrm{MBE}$ system and then gold is deposited for a capping layer. The magnetron sputter is started with a base pressure of $\approx 10^{-10}$ Torr, then an ultra-pure argon source is bled in, bringing the deposition pressure up to $10^{-3}$ Torr. All gauges and gas flow are monitored in real time to spark a stable plasma in the chamber in order for deposition to start; angle and distance from source are also controlled to adjust speed of metal growth. After metal deposition is finished gaseous lines are cut off and UHV pumps are turned back on to transfer the sample to the MBE chamber. The MBE chamber has a base pressure of $\approx 10^{-11}$ Torr and uses standard Knudsen cells for metal deposition. All metals used in the MBE are listed in Table 3.1.

\subsubsection{Sample mounting}

Shortly after metal deposition has taken place, the freshly-fabricated device is mounted to our custom STM BEEM plate shown in Fig. 3.2. For BEEM measurements the top contact of metal and the backside contact on the semiconductor must be separately contacted. Each contact needs to produce an ohmic response to guarantee 


\begin{tabular}{lllllll}
\hline & \multicolumn{2}{c}{ E-beam } & \multicolumn{2}{c}{ Sputter } & \multicolumn{2}{c}{ MBE } \\
& Temp. (C) & Rate $(\mathrm{nm} / \mathrm{s})$ & Temp. (C) & Rate $(\mathrm{nm} / \mathrm{s})$ & Temp. (C) & Rate $(\mathrm{nm} / \mathrm{s})$ \\
\hline $\mathrm{Au}$ & $\mathrm{n} / \mathrm{a}$ & $\mathrm{n} / \mathrm{a}$ & $\mathrm{n} / \mathrm{a}$ & $\mathrm{n} / \mathrm{a}$ & 1380 & 0.47 \\
$\mathrm{Ag}$ & $\mathrm{n} / \mathrm{a}$ & $\mathrm{n} / \mathrm{a}$ & $\mathrm{n} / \mathrm{a}$ & $\mathrm{n} / \mathrm{a}$ & 950 & 0.14 \\
$\mathrm{Cr}$ & $\mathrm{n} / \mathrm{a}$ & $\mathrm{n} / \mathrm{a}$ & 22 & 0.116 & $\mathrm{n} / \mathrm{a}$ & $\mathrm{n} / \mathrm{a}$ \\
$\mathrm{Cu}$ & $\mathrm{n} / \mathrm{a}$ & $\mathrm{n} / \mathrm{a}$ & $\mathrm{n} / \mathrm{a}$ & $\mathrm{n} / \mathrm{a}$ & 1240 & 0.11 \\
$\mathrm{~W}$ & $\mathrm{n} / \mathrm{a}$ & 0.051 & 22 & 0.03 & $\mathrm{n} / \mathrm{a}$ & $\mathrm{n} / \mathrm{a}$ \\
\hline
\end{tabular}

Table 3.1: Temperatures are measured in degrees $\mathrm{C}$ and rate is calculated in nanometers per minute (nm/min). Metals used for deposition are all $99.99 \%$ pure and bought commercially.

that the low current is able to be measured. For both convenience and functionality to make an ohmic contact with two different materials, indium metal is used. Indium has a low melting point at around $150^{\circ} \mathrm{C}$, which makes it a metal that can be made into a contact with soldering and a hotplate. Its also functions as an ohmic contact to silicon for the semiconductor backside contact to ground. A beryllium copper wire is used in conjunction with the indium for the top metal contact to the STM plate and is labeled in Fig. 3.2 as the STM contact. An ohmic BEEM contact is made by using a diamond scribe to score the backside of the semiconductor and the sample is cold pressed onto the indium. The backside contact electrical connection is to the pico-ammeter and the top metal contact is the electrical path to ground for STM pre-amp. The contacts are done this way to ensure that each are electrically isolated from the other for accurate measurement of the current.

\subsection{Sample Measurements}

\subsubsection{Current-Voltage Spectroscopy}

To ensure that each sample has been mounted properly, current-voltage (IV) measurements are done to test rectification of the device. All devices are measured once at room temperature with a Kiethley 2400 source meter and again at liquid nitrogen temperature of $77 \mathrm{~K}$ in the STM. To measure the device a voltage is swept from $+2 \mathrm{~V}$ to $-2 \mathrm{~V}$ with a resolution of $40 \mathrm{meV}$ and a current is measured. The IV curve generated is indication of how well the diode was constructed and some of its properties. From the IV, the slope of the curve and the turn on voltage is observed. 


\subsubsection{Ballistic Electron Emission Microscopy}

All experiments presented have been measured using the BEEM technique. BEEM is a novel technique to probe the electrostatics of the interface with nanoscale precision. Measurement of the Schottky barrier, elastic scattering, charge density fluctuation, and inelastic collisions can be measured. BEEM uses hot carriers injected from an atomically sharp STM tip into the metal. The type of carrier can either be electrons or holes depending on the type of semiconductor and sign of the applied bias. Four types of measurements are done with the BEEM technique and they will be discussed.

BEEM requires a specific configuration of grounded contacts to measure the Schottky barrier of the device. As described in the mounting procedure, the top metal contact is grounded to the STM and the backside of the semiconductor is connected to the Stanford Research Systems SR 570 pico-ammeter. The STM is kept in constant feedback mode to monitor the tip position and magnitude of the current being injected. The feedback loop keeps the tunneling stable during BEEM measurements. A voltage bias is applied between the STM tip and the metal and is swept from $\pm 0.2 \mathrm{~V}$ to $\pm 2 \mathrm{~V}$ depending on the measurement type being performed. Any electrons that have enough momentum to overcome the barrier height of the

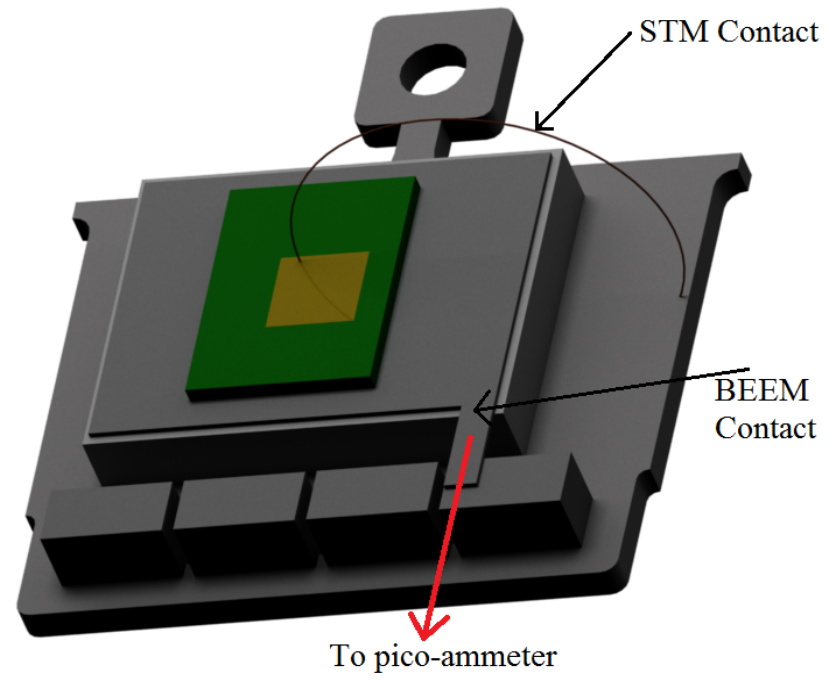

Figure 3.2: Diagram showing the wiring of a BEEM plate. Metal and semiconductor both have separate contacts with preamplifier reading current from the semiconductor with bias placed on the tip. 
specific metal-semiconductor are collected by the backside contact as BEEM current as shown in Fig. 3.3.

One type of measurement done with BEEM is called forward BEEM. When the bias on the STM tip is applied with a positive voltage to the metal, electrons are able to tunnel from the tip to the metal. When the substrate is n-type and grown with donors, the electrons with energy greater than the barrier height is able to be collected as BEEM current. This is shown in Fig. 3.4. Reverse BEEM is performed when the majority carrier in the semiconductor is opposite of the injected carrier from the tip. i.e. if the majority carrier in the semiconductor is electrons, the tip is injecting holes into the metal with a negative applied bias.

The final configuration is forward BHEM where a negative applied bias is used to inject holes into the metal. If the majority carrier in the semiconductor is ptype, a negative applied bias has holes being injected into the metal and when the hole has energy greater than that of the barrier height of the metal/semiconductor junction, it is collected as BHEM current. This is shown in Fig. 3.5. Reverse BHEM is the configuration when a positive bias is applied between the tip and the metal with a p-type semiconductor. In this setup electrons tunnel into the metal, and an

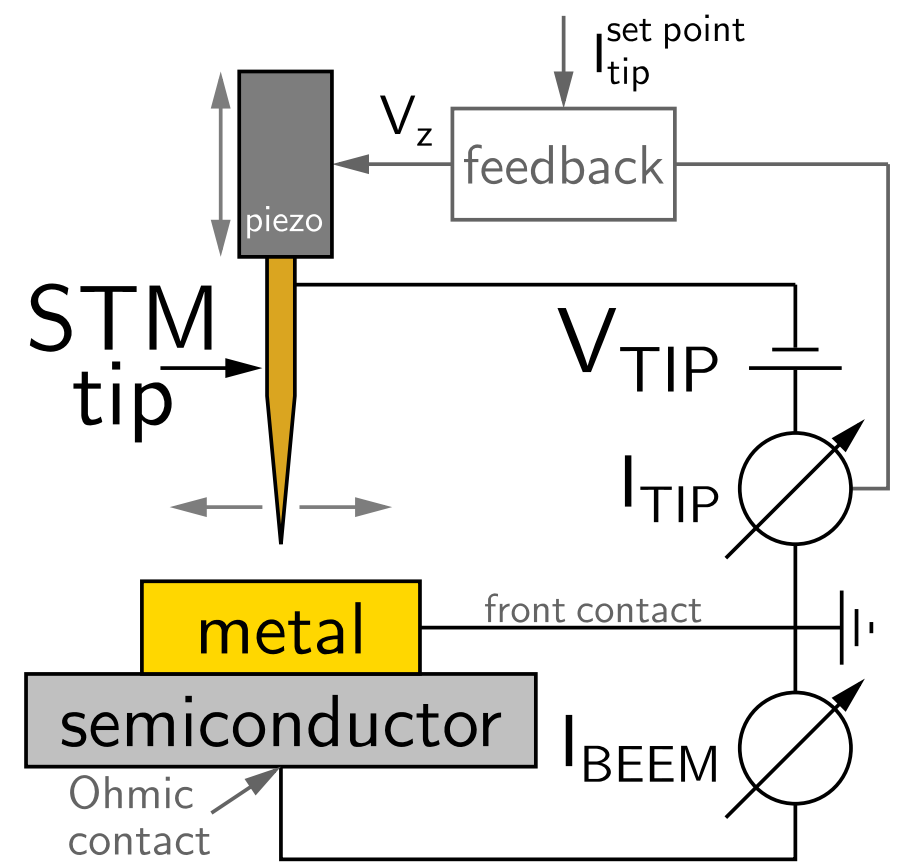

Figure 3.3: Diagram of the wiring for BEEM setup. Both the Metal and semiconductor are grounded; with a preamplifier reading current from the semiconductor while a bias placed on the tip to the metal. 


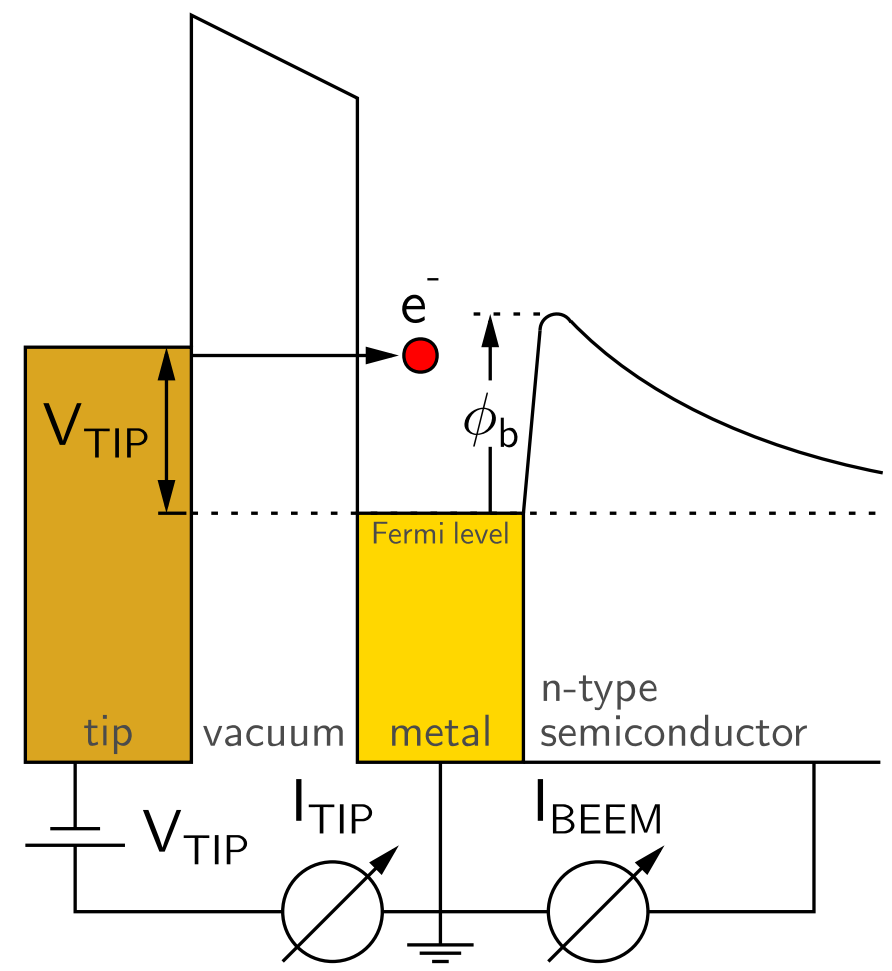

Figure 3.4: Energy band diagram of the BEEM setup, showing the STM tip on the left, metal film in the center and the n-type semiconductor on the right.

electron hole pair excitation generates holes that are able to conduct in the p-type semiconductor. For the measurements done in this work forward BEEM and BHEM are only used for measurements.

A low temperature UHV STM (Omicron) was modified to be used for all BEEM and BHEM measurements with a base pressure in the $10^{-11}$ mbar range [85]. All samples were loaded in the UHV environment and on to the liquid nitrogen cooled, $78 \mathrm{~K}$ STM stage within 3 hours of deposition, and then BEEM and BHEM measurements were performed.

\subsubsection{BEEM Mapping}

Each BEEM spectra was acquired at unique tip locations every $10 \mathrm{~nm}$ to $11.7 \mathrm{~nm}$ on a grid over a $1 \mu \mathrm{m} \times 1 \mu \mathrm{m}$ area by sweeping the voltage from $0.2 \mathrm{~V}$ to $2 \mathrm{~V}$ for the $n$-type diodes, and $-0.2 \mathrm{~V}$ to $-2 \mathrm{~V}$ for the $p$-type diodes. Between 7225 and 10000 spectra are collected over a 36 hour period. An offset current was set with an SR 570 pico-ammeter to zero out any noise. All measurements were taken in absence of ambient light. Each spectra taken across this grid is fit the BEEM model using the 


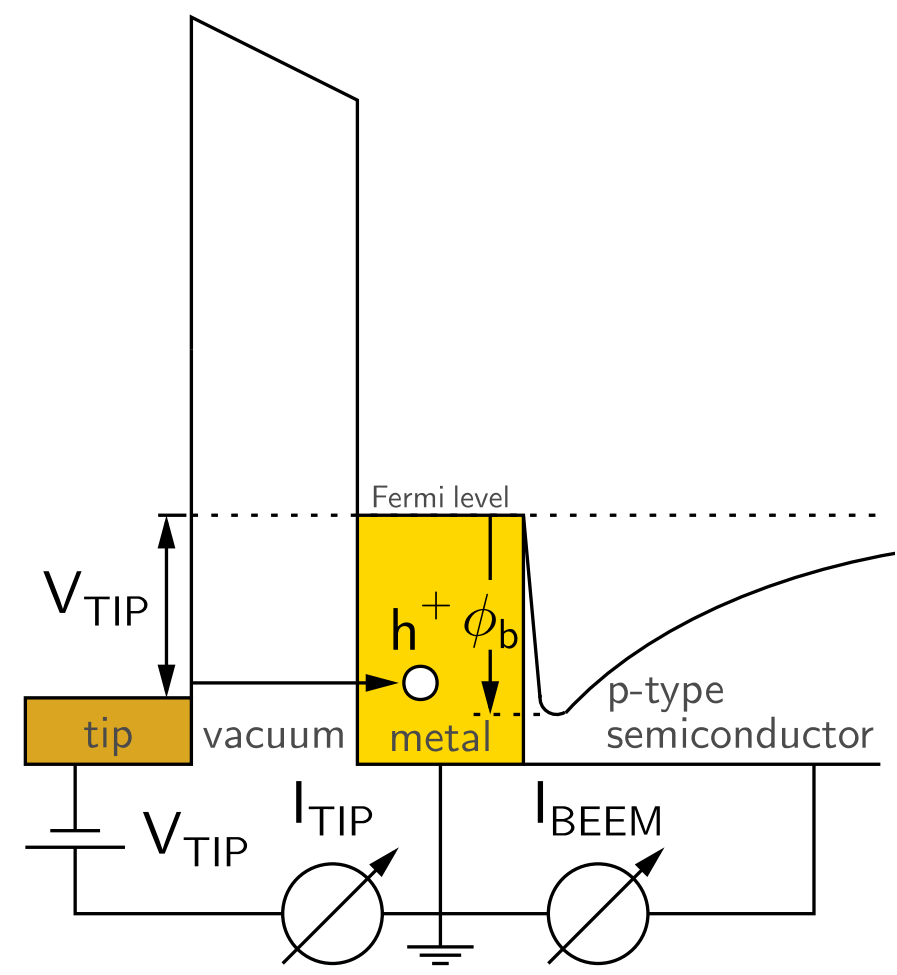

Figure 3.5: Energy band diagram of the BHEM setup, showing the STM tip on the left, metal film in the center and the p-type semiconductor on the right.

routine described in Chapter 2. The barrier height is extrapolated and is binned into a histogram of threshold values. The histogram is binned based on the resolution of the points in the spectra.

An evenly spaced grid as shown in Fig. 3.6, enables a systemic collection of spectra over the $1 \mu \mathrm{m}^{2}$ area. Each grid location and its corresponding spectra can then by mapped and colored according to the value of the threshold from the fit to the spectra at that grid location. The false color imaging provides a visual indication of changes of the threshold across the interface where the human eye can easily detect changes and trends across the entire $1 \mu \mathrm{m}^{2}$ area. 


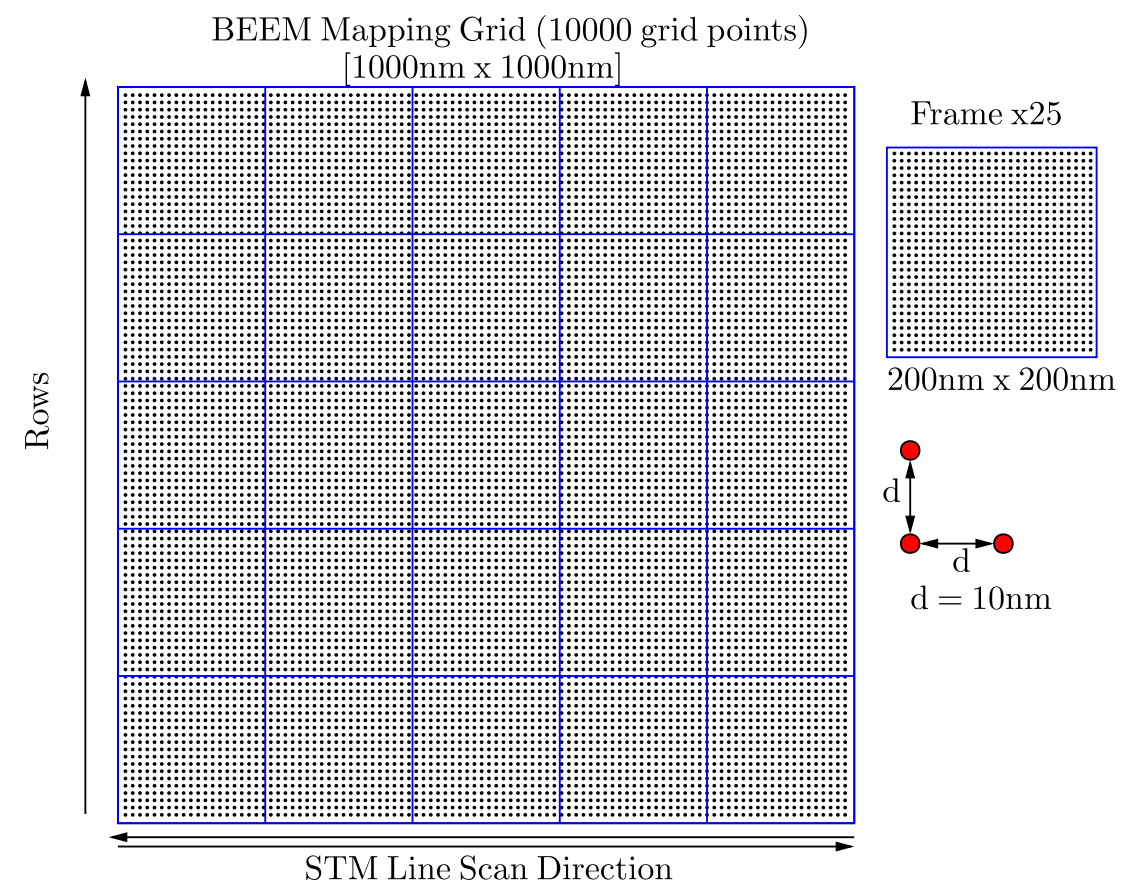

Figure 3.6: BEEM Mapping grid. 10000 spectra are taken over a $1 \mu \mathrm{m}^{2}$ area region. The region is broken down into 25 individual frames with data taken every $10 \mathrm{~nm}$. 


\section{CHAPTER 4}

\section{Nanoscale Schottky barrier mapping of thermally evaporated and sputter deposited $\mathrm{W} / \mathrm{Si}(001)$ diodes and the presence of multiple Schottky barriers}

\subsubsection{Results}

The cross-sectional TEM image of the sputter sample shows distinct regions of metal with abrupt interfaces between the $\mathrm{Au}, \mathrm{W}$, and $\mathrm{Si}$ and is displayed in Fig. 4.1(c) and TEM images of the e-beam deposited samples display an intermixed region of $\mathrm{W}$ and $\mathrm{Si}$ and have been previously published [42, 43]. The averaged BEEM spectra and corresponding fits indicating the Schottky barrier height for all four samples are plotted linearized and displayed in Fig. 4.2. Each spectra is an average of 7225 spectra and fit to $n=5 / 2$, with an $R^{2}$ value of 0.999 or greater. The barrier heights for both the $\mathrm{p}$ and n-type sputter samples are both about $0.07 \mathrm{eV}$ less than the ebeam deposited samples. The solid line indicates the region of fit $(0.2 \mathrm{eV})$ and the dotted line indicates the extrapolation of the fit to the x-axis intercept or $\phi_{b}$. The extrapolation region is smallest $(0.02 \mathrm{eV})$ for the n-type sputter sample and larger for the other samples $(0.05-0.07 \mathrm{eV})$. All four average spectra are plotted together in Fig. 4.3 to demonstrate the shift in the barrier heights and similarities in the sum of the barriers. Fits using $n=2$ were also performed resulting in a sum of barrier heights that was $0.07 \mathrm{eV}$ higher than the band gap of silicon and not considered any further.

The spatial map of the Schottky barrier height obtained from the fitting of each individual spectra for all four samples are displayed in Fig. 4.4. The maps of the sputter deposited samples show a very homogeneous distribution of Schottky barrier heights, whereas the maps of the e-beam deposited sample show a spatially heterogeneous distribution with several localized regions with barrier heights higher than those obtained from fits to the average.

The histograms of all the barrier heights for all four samples, along with the mean barrier height and standard deviation of the distribution, are plotted in Fig. 4.5. The histograms for the sputter deposited samples show a symmetric distribution 


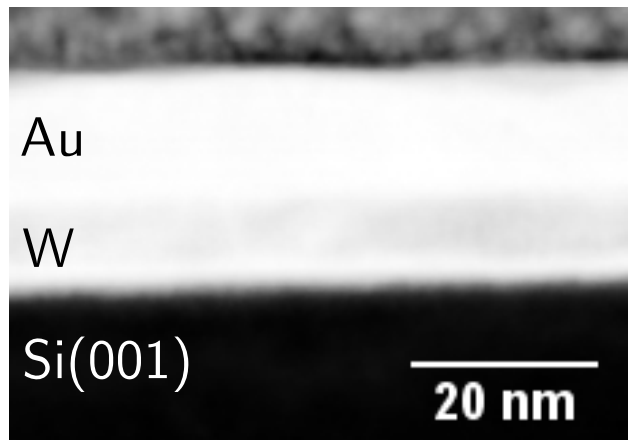

Figure 4.1: TEM image of sputter deposited tungsten/Si sample. An interfacial layer of tungsten and tungsten silicide is formed.

with a mean barrier height very close to the barrier height obtained from the fit to the averaged spectra. The distribution of the n-type sputter sample is the narrowest and this increases for the p-type distribution. The distributions for the e-beam samples show a much broader distribution when compared to the sputter samples and are asymmetric with a long tail to higher (lower) energies for the n-type (p-type) samples. The sputter samples mean barrier height is higher (lower) than the Schottky barrier height obtained from the fit to the averaged spectra for the n-type (p-type) samples. A Monte-Carlo model was developed to simulate the histogram based on adjustable elastic $\Gamma_{e}$ and inelastic scattering rates $\Gamma_{i}$ as well as a Gaussian distribution $\sigma_{\phi_{b}}$ of barrier heights at the interface. Its results are plotted in Fig. 4.6 and is discussed in section 4.1 .

The Schottky barrier heights are plotted vs. $\mathrm{R}^{2}$ value for all the individual fits along with lines indicated the mean $\mathrm{R}^{2}$ and mean Schottky barrier height, as displayed in Fig. 4.7. The sputter deposited samples show a tight locus of points around the intersection of the average values, whereas the e-beam samples show much more diffuse distribution of points. All the plots show distributions of values about the locus with little trend of correlation between the two, but the e-beam samples do show much lower $\mathrm{R}^{2}$ values. 

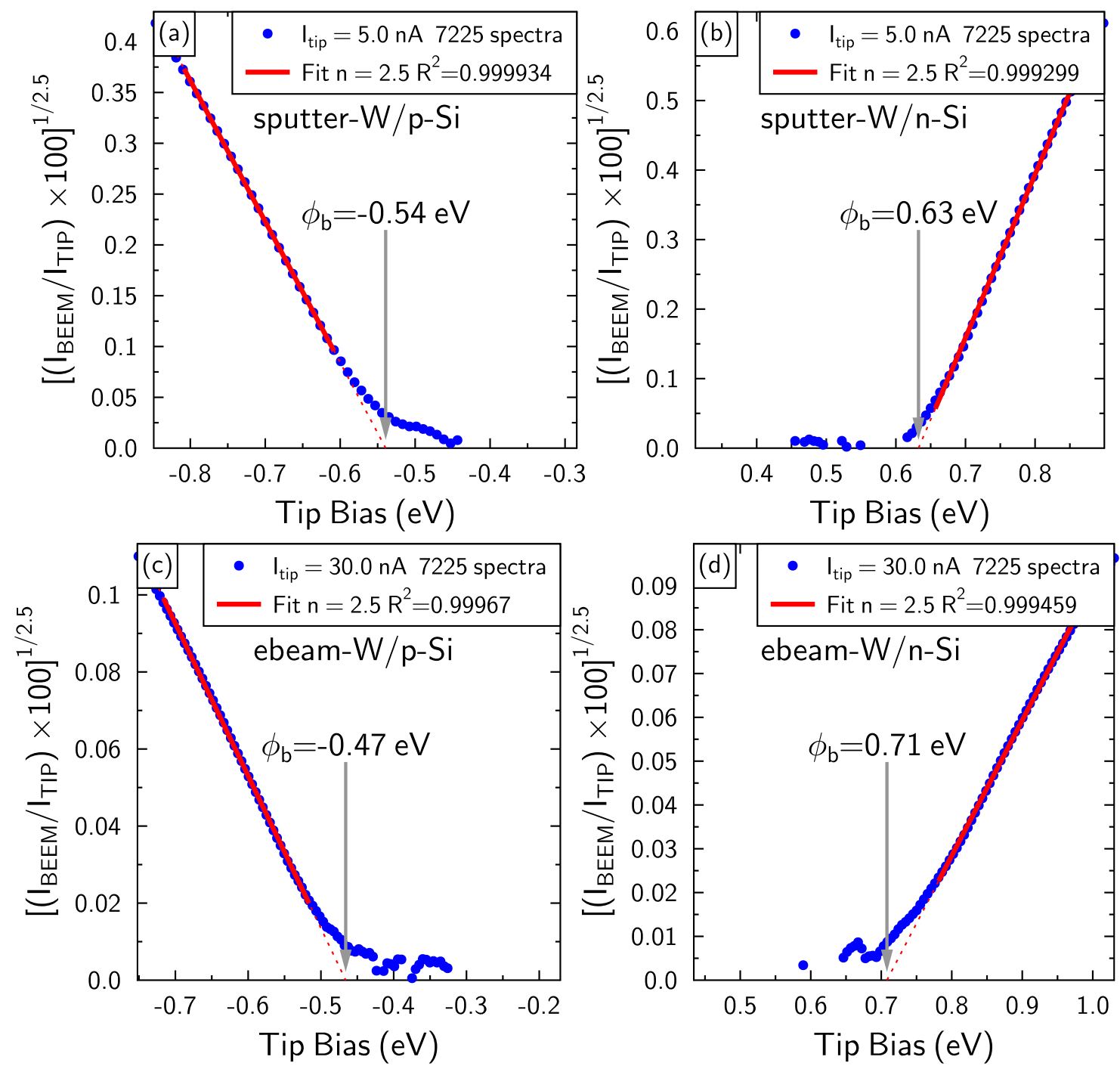

Figure 4.2: (a) Linearized fit of BHEM spectra of p-type sputter Schottky diode using exponent of $n=2.5$. (b) n-type linearized fit of sputter Schottky BEEM spectra. (c) p-type e-beam linearized fit of BHEM spectra showing an increased Schottky barrier height compared to its sputter equivalent. (d) n-type e-beam linearized fit of BEEM spectra.

\subsubsection{Discussion}

Previous IV studies of sputter deposited W/Si(001) Schottky diodes measure barrier heights of $0.65 \mathrm{eV}$ and $-0.5 \mathrm{eV}$ for $\mathrm{n}-\mathrm{Si}$ and p-Si, respectively [86, 87]. These barrier heights are in good agreement with the sputter deposited tungsten samples reported here. Interestingly the e-beam samples show a $70 \mathrm{meV}$ shift to higher energies, which was also observed with sputter and then annealed tungsten [87]. In 
addition, $60 \times$ less transmission is observed in the e-beam samples ( $1 \%$ for sputter to $0.017 \%$ for e-beam at $1 \mathrm{~V}$ ). The most likely cause of this barrier shift and decrease in current for the e-beam samples is silicide formation from unintentional heating during electron beam deposition [88]. Tungsten is a high $\mathrm{Z}$ refractory metal which requires a high emission current output from the electron beam to heat the tungsten source to a high enough temperature for deposition onto the substrate. This would cause unintentional radiative heating of the substrate due to its proximity to the source which would promote intermixing and the formation of a silicide. The TEM images support this finding as they show a more intermixed interface for the e-beam sample [42] when compared to sputter sample, which shows a very abrupt interface as seen in Fig. 4.1. This silicide formation has a dramatic effect on the barrier heights measured by BEEM which is independent of carrier type, as the sum of the $\mathrm{p}$ and $\mathrm{n}$ barrier heights for all samples are in good agreement with the band gap of silicon.

The average spectra and all individual spectra were fit to an $n=5 / 2$ exponent to the simplified BEEM power-law model. In general there are two values utilized in the BEEM model, $n=2$ Bell-Kaiser (BK) and $n=5 / 2$ Prietsch-Ludeke (PL) $[11,24]$. Both models assume no scattering within the metal and the PL model adds energy dependent transmission coefficient for tunneling transport across the Schottky barrier. The PL model systematically lowers the Schottky barrier when compared to the BK model and shows better agreement with the silicon band gap at $78 \mathrm{~K}(1.166 \mathrm{eV}$ for $\mathrm{Si}$ at $78 \mathrm{~K}$ ) for both sets of samples, as indicated in Fig. 4.3 [89]. These samples are formed on low doped silicon that would result in a long depletion width which should inhibit tunneling. However, the thin interface silicide that forms is most likely affecting the characteristic of the threshold region in these BEEM spectra. Image force lowering of the barrier height for these low doped silicon substrates is calculated to lower the sum of both gaps by $0.013 \mathrm{eV}[90,91]$. This lowering does not alter the agreement with the gap significantly or alter the choice of BEEM model, in contrast to $\mathrm{Au}, \mathrm{Ag}$, and $\mathrm{Cu}$ Schottky diodes on the same substrates where the BK model gave the best agreement with the Si band gap [91].

The Schottky maps are a dramatic visual indication of the electrostatic fluctuations of the interface and how the interface depends upon its structure in Fig. 4.4. The maps for the sputter samples are uniform and homogeneous with minimal localized 


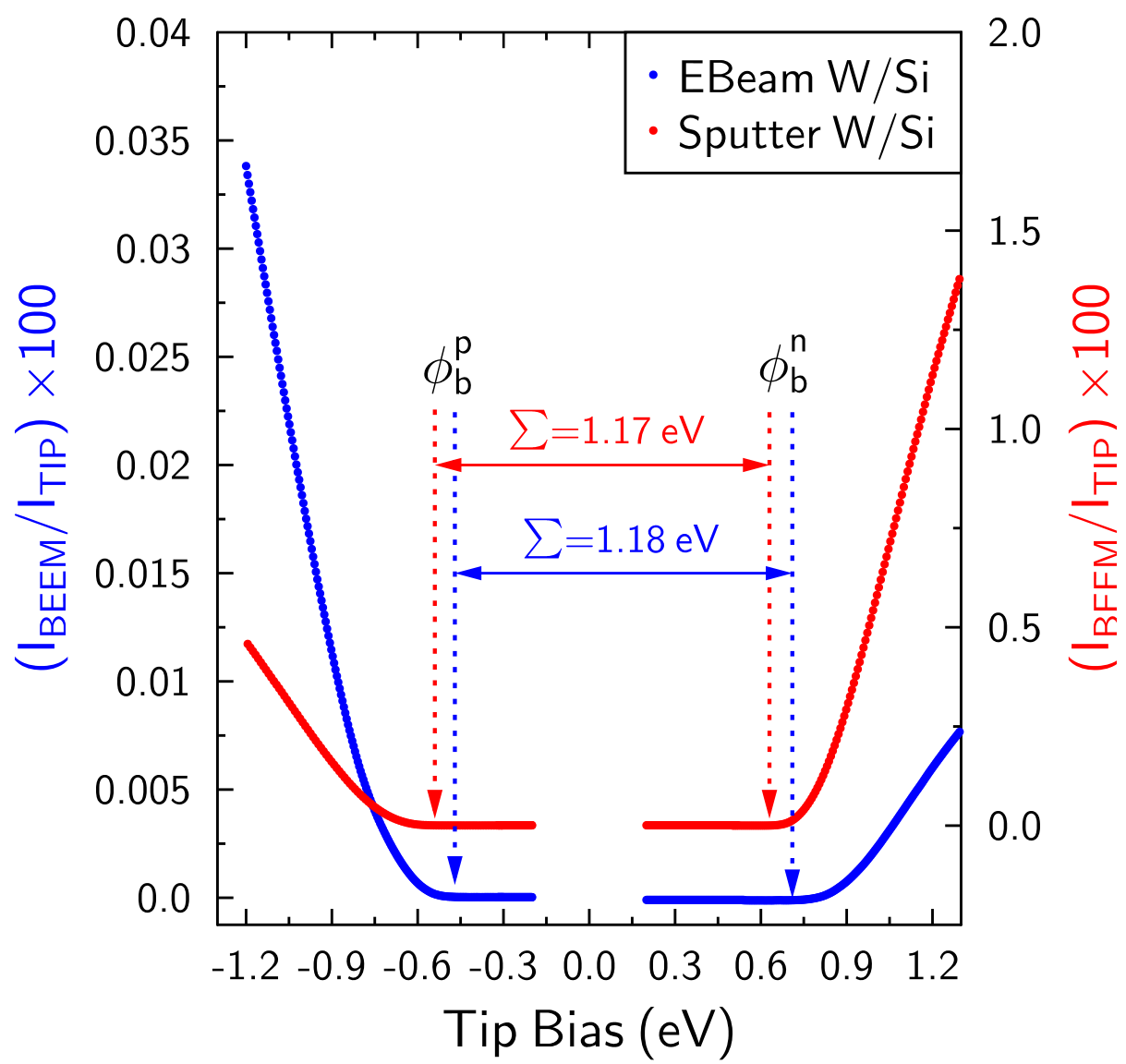

Figure 4.3: Average spectra of all four samples plotted together to demonstrate shift in barrier heights and agreement to the band gap of silicon. Each curve is plotted versus percent transmission of the BEEM current.

defects, whereas the e-beam sample shows a dramatic variation in the map, with large portions at different heights and a high degree of localization. These variations in the barrier height are consistent with the larger degree of interface disorder present in the e-beam sample and can be caused by impurities, structural disorder from incomplete silicide formation, or any other defects that would alter the charge density near the interface $[92,93]$. In addition, elastic scattering of the hot electrons can cause higher thresholds in the BEEM spectra as it forces the electrons to need a greater total energy for transmission and can be supported by looking at the histograms of the barrier heights.

The symmetry, distribution width, and mean of the histogram of barrier heights contain a tremendous amount of information about the electrostatic character of the interface as a whole, as seen in Fig. 4.5. The most symmetric histogram is for n- 

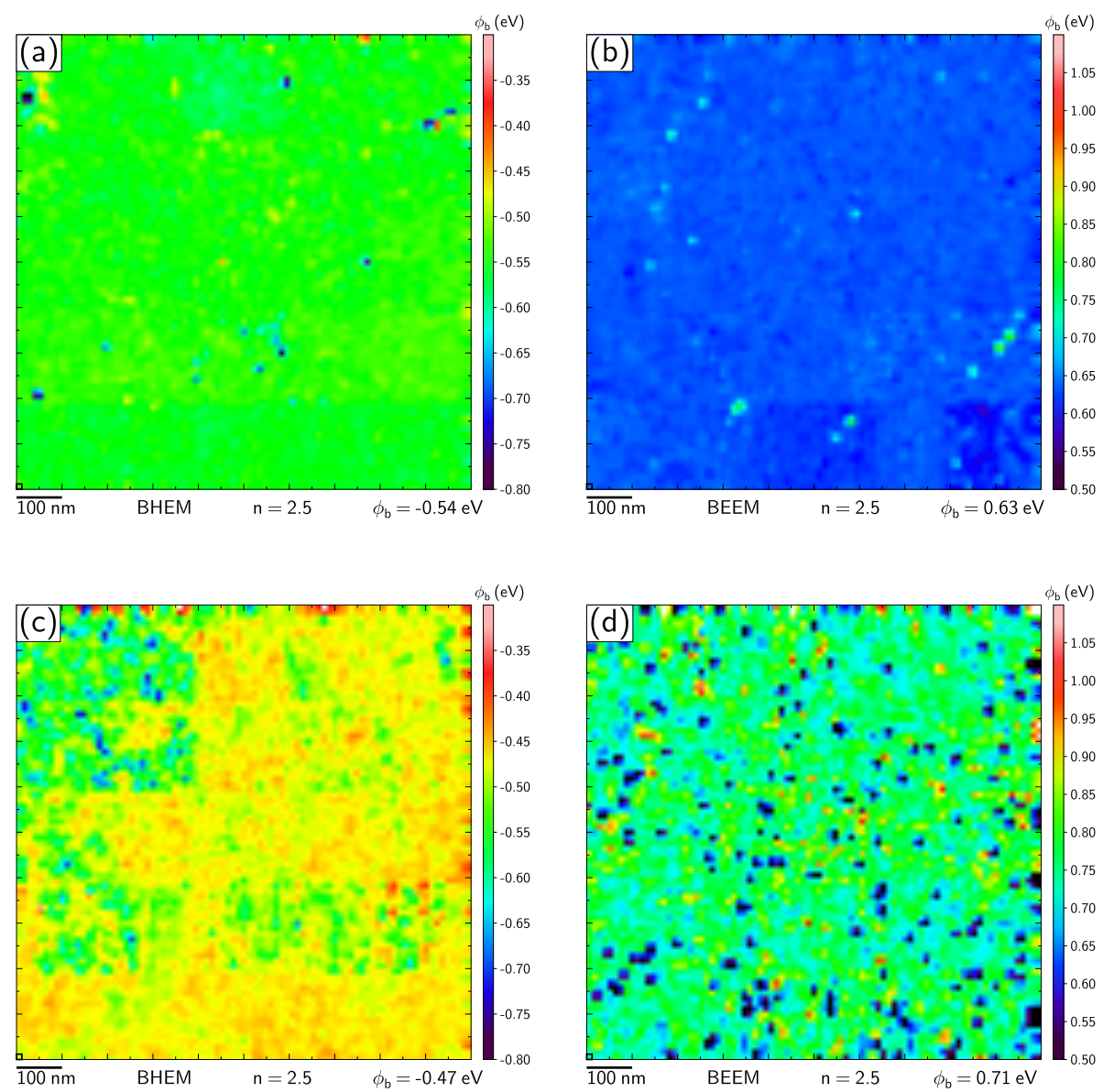

Figure 4.4: Each map displayed is representative of the electrostatic interface. (a) and (b) BHEM and BEEM measurements show a uniform interface for sputter. (c) and (d) e-beam samples show a very disordered interface caused by a change in the interface chemistry during deposition.

type sputter sample. It has the smallest $\sigma$ and the mean of the distribution is equal to the fit to the average $\left(\bar{\phi}_{b}=\phi_{b}\right)$. The corresponding fit to the average has the smallest extrapolation region, which arises from the narrow distribution as seen in Fig 4.2(b). The p-type sputter sample also exhibits similar features but with a slightly larger $\sigma$ and very small $(0.01 \mathrm{eV})$ difference between $\bar{\phi}_{b}$ and $\phi_{b}$. The extrapolation region of the fit to the average is also larger for the p-type sputter sample as seen in Fig. 4.2(a). The e-beam samples are dramatically different with much greater $\sigma$, a systematically lower mean Schottky obtained from a fit to the averaged spectra, and highly asymmetric distributions. Both the extrapolation regions of the fits to the average spectra are large and similar to the p-type sputter sample as seen in 

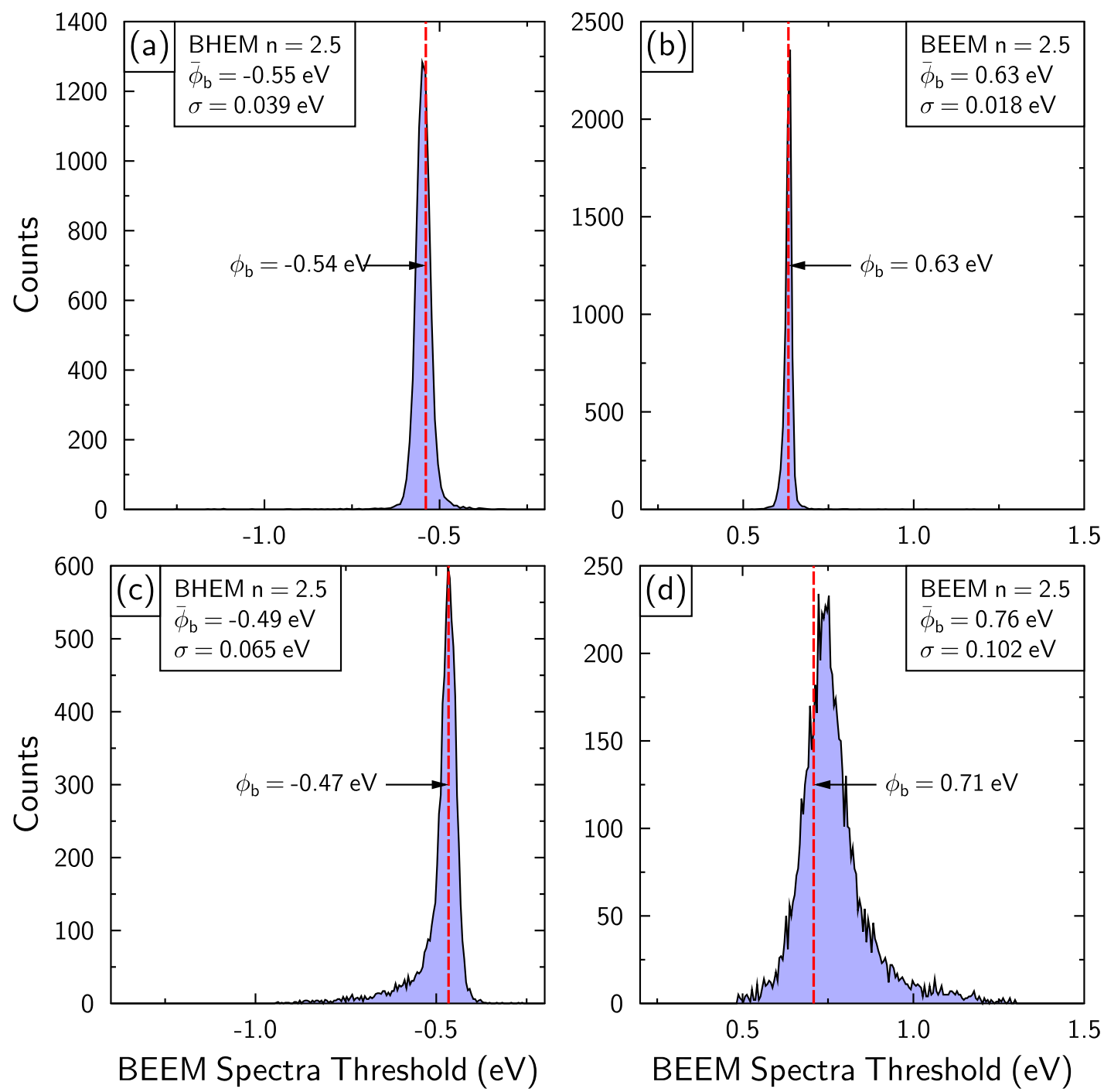

Figure 4.5: Histogram of Schottky barrier heights from fits to the individual spectra. (a) \& (b) are for the sputter sample and show a symmetric and narrow Gaussian-like distribution and good agreement between the mean Schottky and fit to the average. (c) \& (d) are for the e-beam sample showing a skewed asymmetric distribution attributed to elastic scattering of the hot electrons.

Fig. 4.2(c)\&(d). The extrapolation region arises from the width of the leading edge of the distributions, which is similar for these three samples. The averaging of multiple spectra with different onsets causes the average spectra to deviate from the linearity near the threshold and results in a fit to a Schottky barrier that is lower than the mean of the distribution, which is calculated from the fits to individual spectra. 


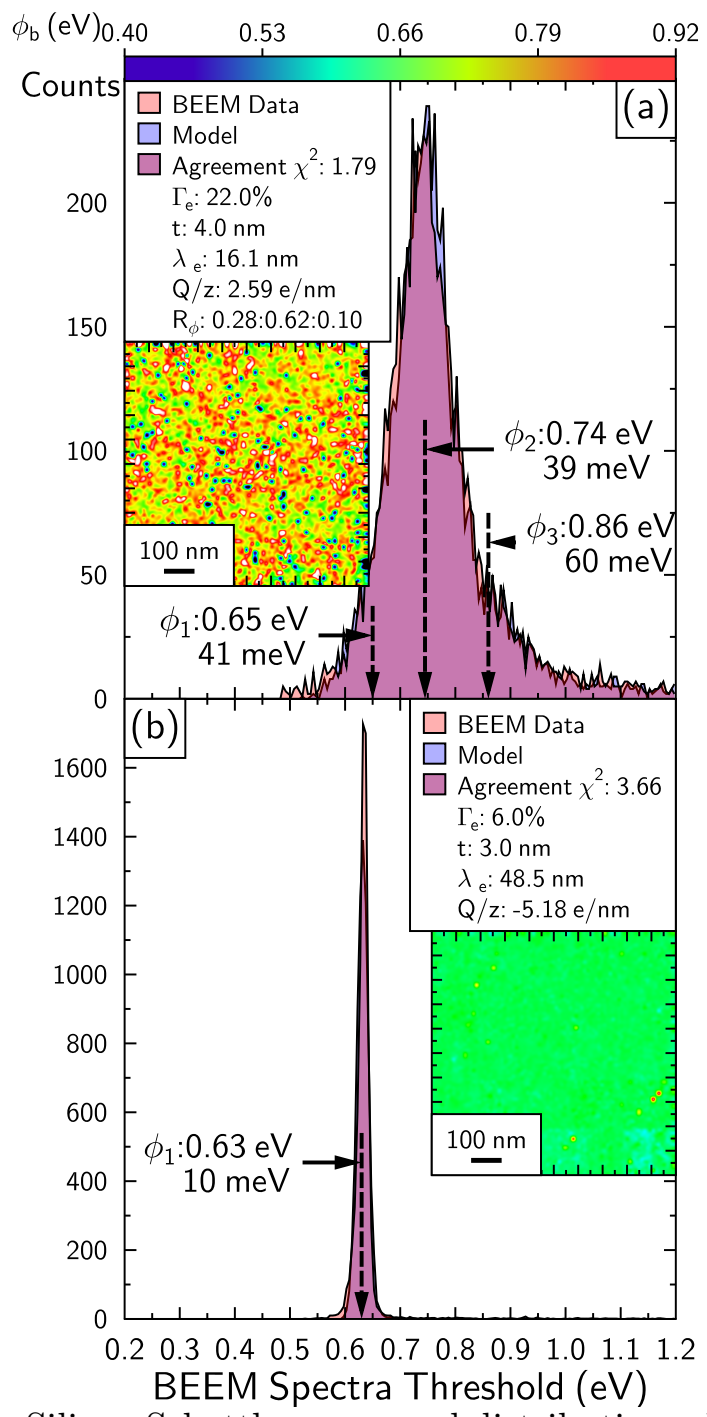

Figure 4.6: Tungsten-Silicon Schottky maps and distributions show the difference between an incomplete silicide formation in (a), and a uniform W/Si interface in (b). The Schottky maps for each show the distribution of threshold values over a $1 \mu m^{2}$

A symmetric Gaussian-like distribution is expected in barrier heights for metal semiconductor systems arising from variations in the interface structure where models based on interface dipoles have predicted this behaviour and have been observed with BEEM $[94,58]$. This is partially the cause of the wider distributions of the e-beam samples as the incompletely-formed silicide would have larger variations in stoichiometry and structure at the interface, resulting in a broadening of the distribution of barrier heights. However, this effect cannot account for the long tail and asymmetry and tail in the distributions for the e-beam samples. The asymmetry in the distribu- 
tion is attributed to elastic scattering which reduces the perpendicular momentum of the hot electrons, causing them to need a higher total energy to overcome the barrier. In contrast, few scattering events exist that would cause the electrons to need a lower total energy. This results in a skewed lognormal like asymmetrical distribution.

To further enhance this argument, a kinetic Monte-Carlo model was developed and utilized which produces a histogram of barrier heights to compare to the measured values. For n-type e-beam sample $\Gamma_{e}=22 \%$ and $t=4 \mathrm{~nm}$ give the best fit to the BEEM data and for the p-type sample these values are $\Gamma_{e}=6 \%$ and $t=3 \mathrm{~nm}$ as indicated in Fig. 4.6. Skewing is not observed if $\Gamma_{e}=0$, indicating that the observed skewing and high energy tail in the BEEM data arise from localised elastic scattering. The localized nature of BEEM and the recording and fitting of individual spectra from a uniformly spaced grid provides this insight which is obfuscated in the averaged spectra.
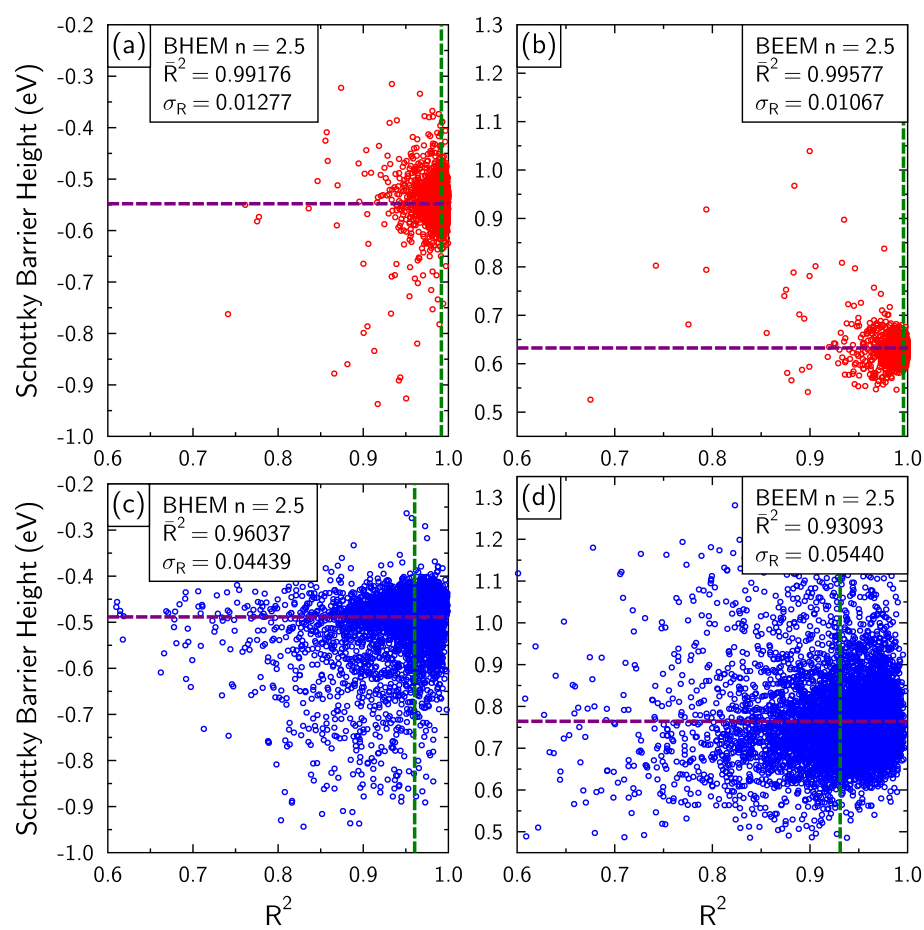

Figure 4.7: $R^{2}$ vs Schottky barrier height for all samples. In the case of sputter (a) and (b), the Schottky barrier heights fits are associated with a tight distribution around the average $R^{2}$ value. Both (c) and (d) e-beam samples exhibit a broad distribution of $R^{2}$ values which correspond to a wider range of Schottky barrier heights.

The plots of the $R^{2}$ values of the fits versus Schottky barriers further support 
the observation that the disorder in the e-beam sample is affecting the interface electrostatics, as shown in Fig. 4.7. For the p-type e-beam sample the width of the $\mathrm{R}^{2}$ distribution is over 2 times greater than the sputter sample as well as 5 times greater for the the n-type samples. However in each sample little correlation is seen between both parameters indicating that high quality fits are obtained throughout the range of barrier heights observed [44].

\subsection{Multiple Barrier Height Modelling}

The dramatic difference between the e-beam and sputter deposited W/Si samples arises from unintentional radiative heating of the sample during e-beam deposition, which does not occur during sputter deposition [44]. The heating promotes silicide formation which has been shown to shift the tungsten-silicon barrier height by roughly $70 \mathrm{meV}[86,87]$. This is captured in the computational modelling which indicates that only one barrier height is needed for the sputter sample, whereas three are needed to describe the e-beam sample. The lowest barrier height $\phi_{1}$ is attributed to $\mathrm{W} / \mathrm{Si}(001)$ with a $28 \%$ contribution, where $\phi_{1}^{\text {sputter }} \approx \phi_{1}^{\text {e-beam }}$. The middle barrier height $\phi_{2}$ is about $90 \mathrm{meV}$ higher and attributed to $\mathrm{WSi}_{2} / \mathrm{Si}(001)$ and is the strongest contributor to the histogram at $62 \%$. The highest barrier height, $\phi_{3}$ is in good agreement with the $\mathrm{Au} / \mathrm{Si}(001)$ barrier height and attributed to gold with only a small 10\% contribution [91]. Fig. 4.1 showed TEM and EDX profiling of a $10 \mathrm{~nm}$ wide cross section of the e-beam sample, showing a significantly intermixed tungsten-silicon region when compared to the sputter sample, which showed an abrupt tungsten to silicon transition $[42,44]$. The presence of a small amount of gold in the model suggests that either gold is in contact with the silicon or that a sub-nm-thick tungsten layer might not be able to "screen" the gold from affecting the barrier height in some regions. Grain boundaries or pin-holes in the tungsten film that formed during the deposition and subsequent heating may offer potential sites for the gold to contact the silicon.

An exhaustive search over seven different simulation runs with barrier heights from one to three, with and without Coulomb scattering, was utilized to arrive at the best-fit three barrier height model for the e-beam tungsten data, as summarized in Fig. 4.8. Previous published results of a simplified model with a fixed barrier height distribution and a single adjustable elastic scattering parameter is displayed 
Fig. 4.8(a). This resulted in a high $\chi^{2}$ but demonstrated that the skewing was caused by the elastic scattering and was the catalyst for developing the more detailed model. One of the biggest impacts in the goodness of fit is the elastic and inelastic scattering during the hot-electron transport through the metal layer. Sources of elastic scattering are grain boundaries, material interfaces, defects, and impurities [95]. Elastic scattering changes the perpendicular momentum of the electron relative to the metal/semiconductor interface causing the electron to need a higher energy to overcome the barrier, skewing the threshold distributions. Inelastic scattering is primarily electron-electron which dominates at energies above the barrier height and is modeled using Fermi liquid theory as previously described.

When only one barrier height is utilized, large elastic and inelastic scattering rates are needed to broaden and skew the histogram to higher energies. The histogram is also broadened by adding additional barrier heights with different energies and widths. The addition of Coulomb scattering improves the fits slightly as well, as shown by the effective charge per unit depth. This is a small effect that allows electrons to surmount the barrier outside of the specified Gaussian distributions at lower or higher energies for positive and negative impurities, respectively. Physical sources of this effect may include ionized impurity atoms in the silicon near the interface or fluctuations in the charge density due to variations in the bonding and defects at the interface [62]. The lowering of $\chi^{2}$ as parameters are added to the model supports their inclusion in the description of the data by increasing the confidence level above $3 \sigma$ as shown in Fig. 4.9. The additional barriers also reduce and stabilize $\Gamma_{e}$ and $t$. Multiple barrier heights resulting from incomplete silicide formation and gold interacting with silicon is a more plausible reason for the broad distribution of the e-beam sample histogram than high elastic and inelastic scattering rates. It should also be noted that both model fits of 3-barrier height with and without Coulomb scattering were above the $3 \sigma$ confidence level. This indicates that while Coulomb scattering does lower the $\chi^{2}$ value both models are considered acceptable.

The modelling of the $\mathrm{Au} / \mathrm{Ag} / \mathrm{Si}(001)$ measurements also provides insight into the structural composition of the interface as displayed in Fig. 4.10 [41]. Au and $\mathrm{Ag}$ are miscible and intermixing of the two metals at the interface would allow both barrier heights to be measured $(\mathrm{Ag} / \mathrm{Si}: 0.67 \mathrm{eV} \& \mathrm{Au} / \mathrm{Si}: 0.85 \mathrm{eV})$ [91]. The best 

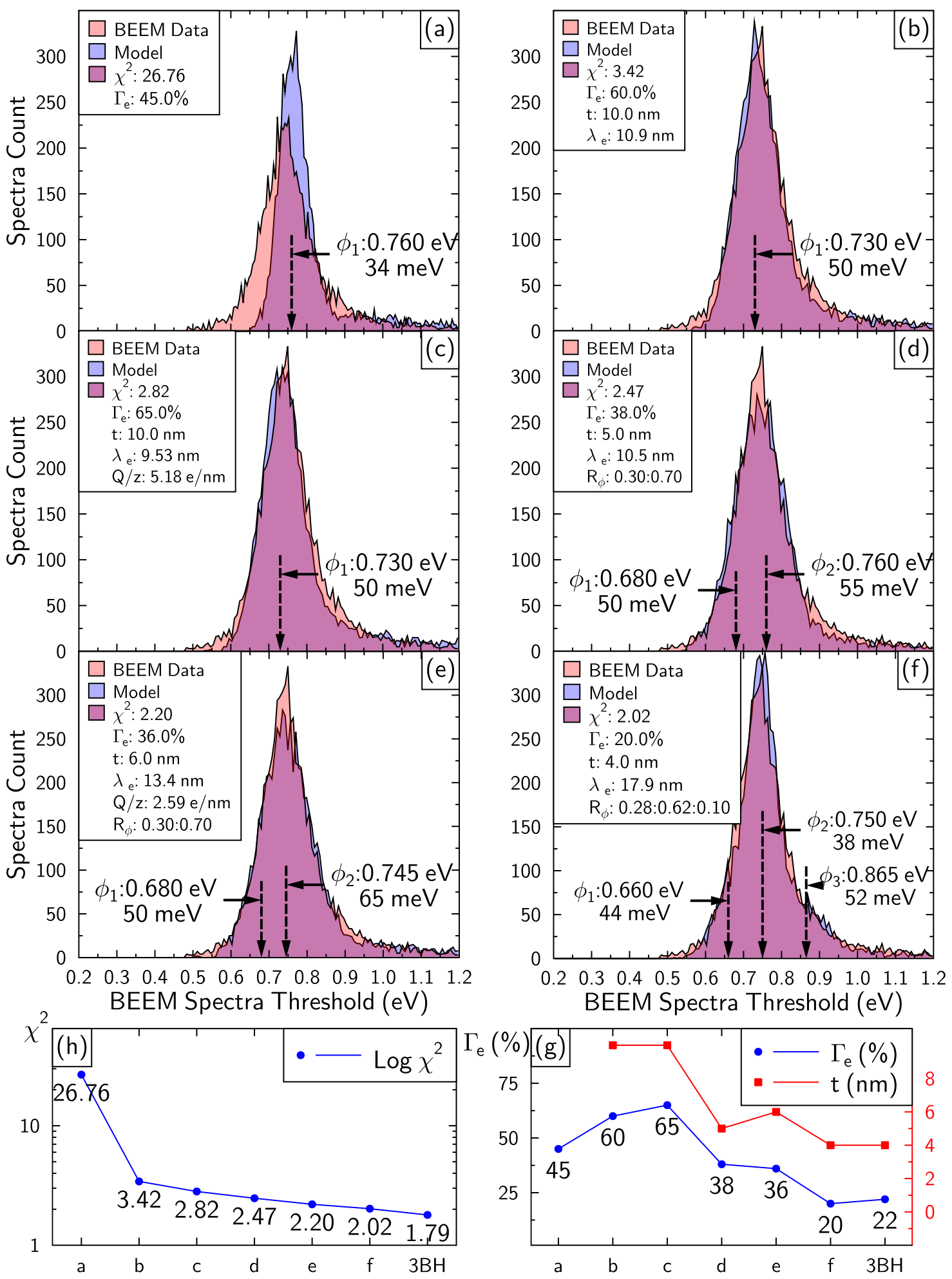

Figure 4.8: The effects of model parameters and number of barrier heights. The progression of $\chi^{2}$ and scattering rate as parameters are added are shown.

fit was found with two barrier heights, where the addition of Coulomb scattering did 


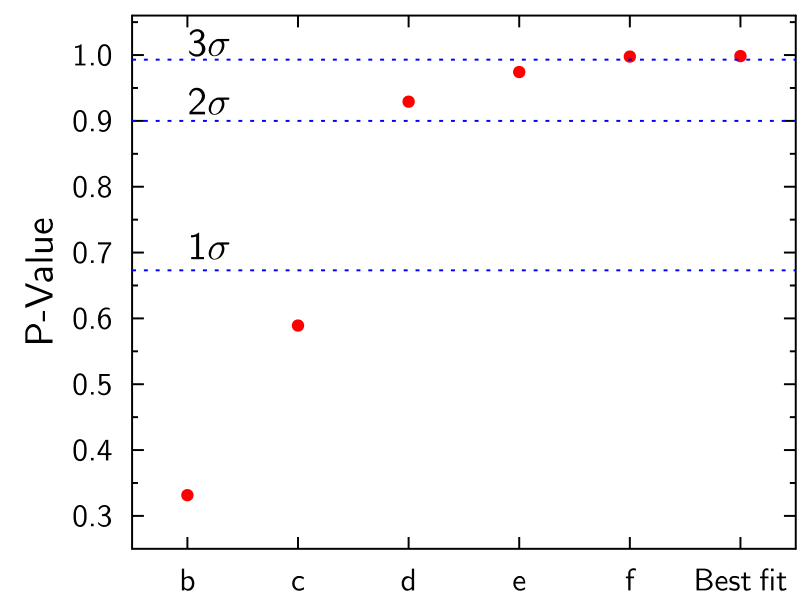

Figure 4.9: Tungsten-Si computational modeling confidence levels from P-value tests using the incomplete gamma function. Best fits are obtained when modeling reaches a $3 \sigma$ value for $\mathrm{P}$-value tests. $\mathrm{x}$-axis values correspond to modeling shown in Fig.4.8.

not significantly improve the fit. For both distributions of the thick (a) and thin (b) $\mathrm{Au} / \mathrm{Ag}$ samples, $\phi_{2} \approx 0.85 \mathrm{eV}$ corresponds to a $\mathrm{Au} / \mathrm{Si}$ and the lower $\phi_{1}$ to $\mathrm{Ag} / \mathrm{Si}$. The barrier height $\phi_{1}$ found in the model is slightly higher than pure $\mathrm{Ag} / \mathrm{Si}$ and may result from alloying or pinch off effects. The barrier height of Au-Ag alloy varies linearly with alloy composition and pinch off effects occur when a nanoscale size region of one metal is surrounded by another of different barrier height [58, 40, 96]. The included region's barrier height is altered due to the continuity of the electrostatic potential at the interface $[41,91,97,71]$. The modeling predicts a $\mathrm{Ag}$ :Au barrier height ratio of 90:10 for the $30 \mathrm{~nm}$ thick silver and 18:82 for the $5 \mathrm{~nm}$ silver. The amount of silver at the interface scales with silver thickness and these ratios are consistent with results from Auger depth profiling [41].

The elastic and inelastic scattering parameters $\Gamma_{e}$ and $\Gamma_{i}$ can be utilized to calculate an inelastic scattering length $\lambda_{e}$ from the probability to elastically scatter $\Gamma_{e}=1-\exp \left[-t / \lambda_{e}\right]$ first described in chapter 2. Solving gives $\lambda_{e}=-t /\left(\ln \left(\Gamma_{e}\right)-1\right)$. This number is calculated for each simulation, included in the figures, and is greater than $t$ or the simulated thickness in all cases. Using Matthiessen's rule $(1 / \lambda=$ $\left.1 / \lambda_{e}+1 / \lambda_{i}\right)$ to calculate the attenuation length at $1 \mathrm{eV}$ for all these samples puts them in the 10-20 nm range, which is similar to lengths measured using attenuation length studies with BEEM [98, 71, 99, 100]. This calculation and the simulations are 


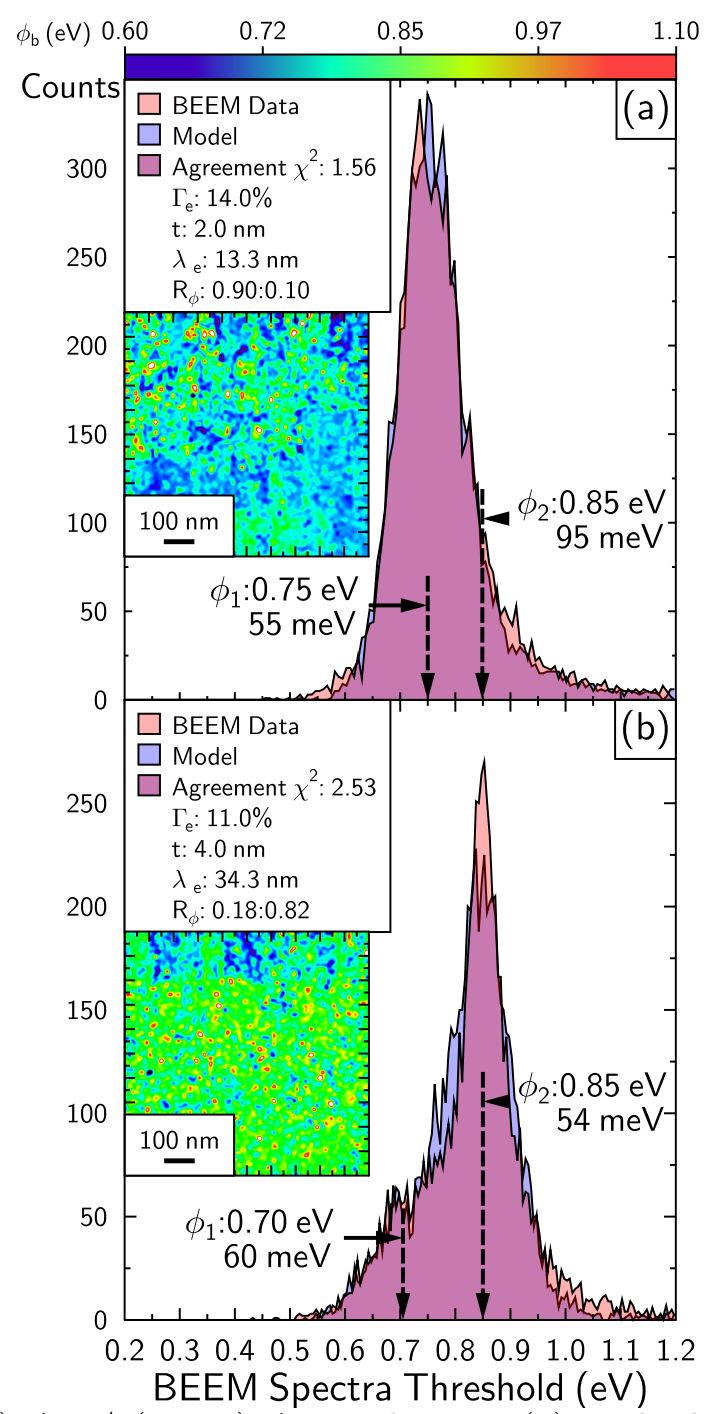

Figure 4.10: $(6.5 \mathrm{~nm}) \mathrm{Au} /(30 \mathrm{~nm}) \mathrm{Ag}$ is show in (a) with the majority of threshold values pinned to a higher energy than the barrier height of $\mathrm{Ag} / \mathrm{Si}$. (10nm) $\mathrm{Au} /(10 \mathrm{~nm}) \mathrm{Ag}$ is shown in (b) with the majority of threshold values equal to the barrier height of $\mathrm{Au} / \mathrm{Si}$.

performed using $a_{o}$ and $E_{F}$ for silver. Using this methodology, along with thickness dependent studies, would provide a means to find these parameters for other metals and a more accurate way to extract the hot-electron-scattering attenuation lengths. 


\section{CHAPTER 5 Study of Chromium Thickness and Silicide Formation Study}

\subsection{Detection of Silicide Formation in $\mathrm{Cr} / \mathrm{Si}(001)$}

The most recent trend in semiconductor manufacturing is to use a controlled growth of a silicide layer between the metal contact and the semiconductor source and drain regions [101, 102]. This controlled growth produces a salicide, or self-aligned silicide layer, on gate-all-around structures or on finFETs as the source and drain regions, as shown in Fig. 5.1. The benefit of using a salicide is to selectively grow an epitaxial layer of metal silicide for lowering the Schottky barrier of the metal contact. Lowering the Schottky barrier is one of the important optimizations to perform in a new process and technology node in order to increase performance or to lower voltage required for operation of the transistor. Transition metals are largely desired for their ability to form silicides and di-silicides easily and at varying temperatures below the eutectic point of silicon.

In this chapter, the transition metal chromium and silicon semiconductor interface are investigated for detection of the formation of a silicide with both n-type and p-type Silicon (001). Chromium is investigated with the BEEM measurement technique and the data is modelled with the computational model discussed in previous chapters.

\subsubsection{Results}

The chromium/silicon Schottky diodes were fabricated using RF magnetron sputtering in a UHV chamber with a base pressure in the $10^{-10}$ mbar range. A $2 \mathrm{~nm}$ chromium layer was deposited on both $\mathrm{n}$ - and p- type high resistivity $(10-100 \Omega \cdot \mathrm{cm})$ single crystal silicon substrates through a $1 \times 2 \mathrm{~mm}$ shadow mask. The doping density of $\mathrm{Si}$ was kept constant throughout all BEEM measurements. Prior to deposition, the substrates were cleaned with a standard hydrofluoric acid treatment to remove the native oxide present on the surface. After Cr deposition the layer was capped in situ with an $8 \mathrm{~nm}$ Au layer to prevent possible oxidation of the Cr layer after removal from the UHV chamber. The thickness of the metal layers were confirmed 

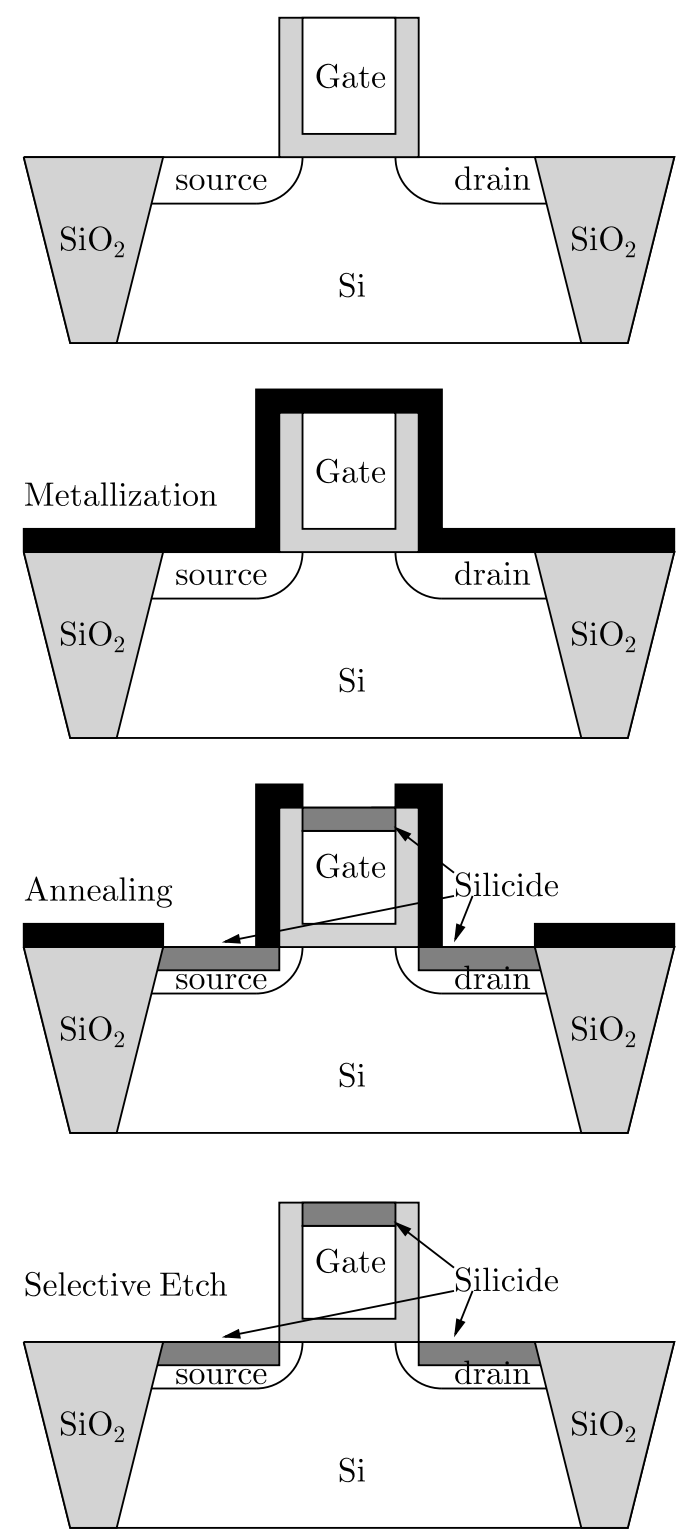

Figure 5.1: Self aligning silicide process contains three major steps: Metallization of the transistor finFET, annealing to promote diffusion of the metal and silicon to grow a silicide, and selective etching of the left-over metal.

ex-situ with ellipsometry as well as transmission electron Microscopy (TEM) and energy dispersive x-ray spectroscopy (EDS) for chemical composition in a FEI Titan $\mathrm{S} / \mathrm{TEM}$ in Fig. 5.3.

The average spectra obtained from the BEEM measurements for both p- and n-type substrates are plotted linearized along with its fit and shows a barrier height of $0.6 \mathrm{eV}$ (p-type) and 0.59 (n-type) in Fig. 5.2(a) \& Fig. 5.2(b), respectively. Both 

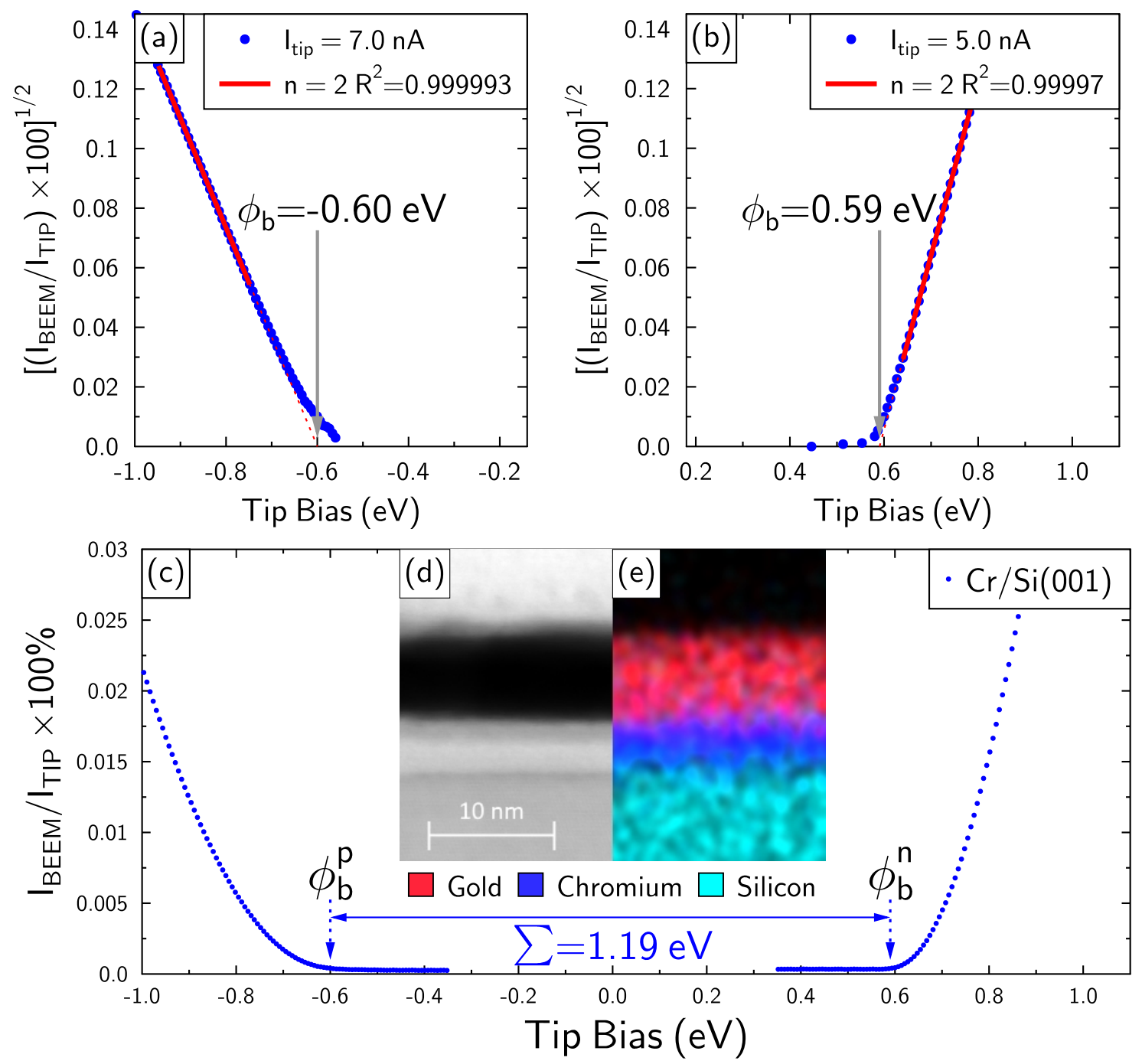

Figure 5.2: (a) Linearized fit of BHEM spectra of p-type $\mathrm{Cr} / \mathrm{Si}$ Schottky diode using exponent of $n=2$. (b) n-type linearized fit of Schottky BEEM spectra. (c) BHEM and BEEM Schottky indicating the summation of the barrier heights. (inset) (d) Bright field TEM image of the $\mathrm{Au} / \mathrm{Cr} / \mathrm{Si}(001)$ interface and (e) corresponding EDS map.

spectrum are plotted together to show that the sum of the barrier heights equal to $1.19 \mathrm{eV}$ in Fig. 5.2 which is close to the $1.17 \mathrm{eV}$ gap of $\mathrm{Si}$ at $77 \mathrm{~K}$ [89]. Image force lowering of the barrier heights for these low doped silicon substrates is calculated to reduce the sum by $13 \mathrm{meV}[90,91]$.

The maps of the barrier height show the spatial distribution of the thresholds are displayed in Fig. 5.4. The map for the p-type substrate shows a high degree of localization with a more disordered interface when compared to the n-type substrate 
that shows a more uniform barrier height map with only a few localized regions. The histograms of the thresholds from fitting to the individual spectra are plotted in Fig. 5.5(a) \& Fig. 5.5(b). The n-type histogram has a narrow distribution of spectra thresholds with a peak at $0.59 \mathrm{eV}$ and a small tail to $0.7 \mathrm{eV}$. In contrast, the ptype histogram has a broader distribution with a peak at $-0.6 \mathrm{eV}$ and a high energy tail extending out past $-1.0 \mathrm{eV}$. The skewing of the distributions to higher energy is the origin of the large extrapolation region observed in the fits to the linearized averaged spectra in Fig. 5.2. This causes the Schottky barrier from the fit to the average spectra to be less than the mean threshold from the distribution as averaging of multiple spectra with different onsets causes the average spectra to deviate from the linearity near the threshold [44]. However, the fit to the averaged spectra gives the best agreement to the band gap of the substrate and is used as the measured Schottky barrier height $[91,44]$.

A Monte-Carlo model is utilized to simulate the histogram of spectra thresholds from individually fit BEEM spectra. These values are an empirical account of the scattering being observed in the measurements and are an indication of the percentage of spectra which exhibit some form of scattering. The modelling is described in Chapter 2.

$R^{2}$ scatter plots show distribution of barrier height fits for the n-type sample localized near $R^{2} \approx 1$ with a standard deviation of 0.0136 from the mean of the distribution. The p-type samples $R^{2}$ have a distribution around $R^{2}=0.964$ and a stadard deviation of 0.0366 nearly 3 times greater than the n-type.

\subsubsection{Discussion}

The Cr/Si(001) Schottky diode has been studied with typical I-V and C-V measurements and report values of $0.60 \mathrm{eV}$ for n-type $\mathrm{Si}(001)$ at room temperature [90]. To the best of our knowledge, values for chromium on p-type silicon have not been reported to date but it can be estimated from the band gap of Si and the n-type value. The best agreement with the gap was achieved with the $n=2 \mathrm{BK}$ model with an $R^{2}$ value of 0.9998 and fits to the PK model, $n=5 / 2$, were performed but resulted in barrier heights sum to $\sim 45 \mathrm{meV}$ less than the band gap of silicon and lower $R^{2}$ values overall. The cross sectional TEM and EDS images of the interface 


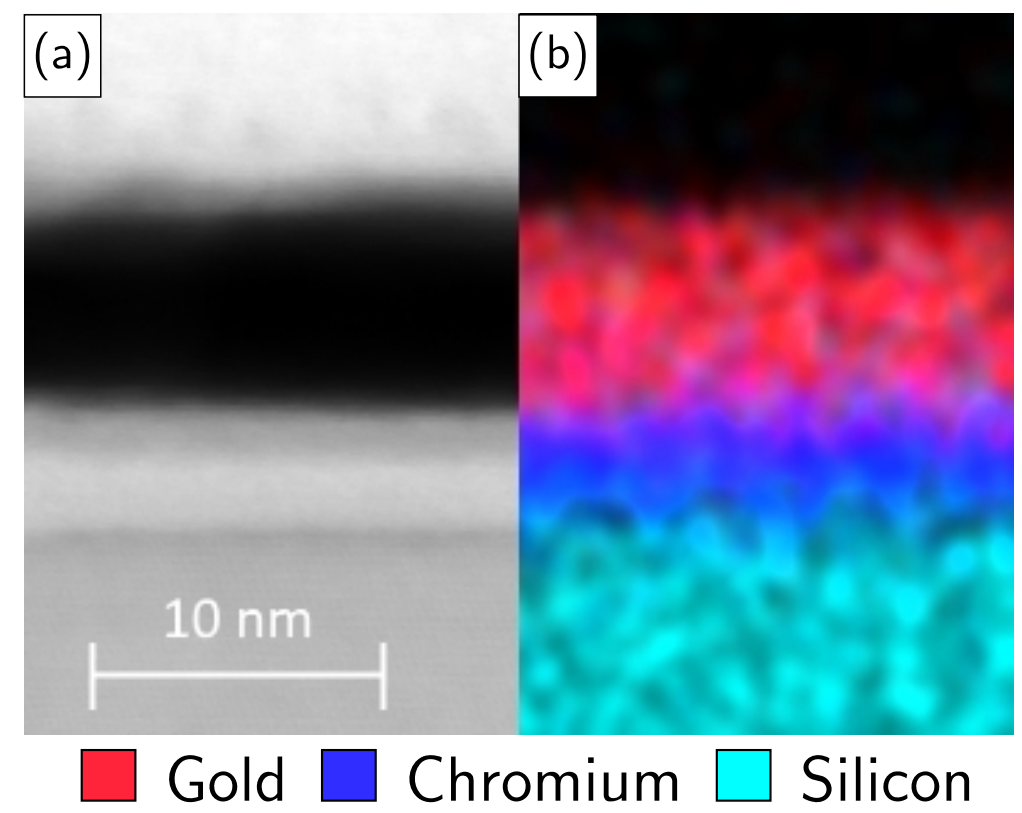

Figure 5.3: Transmission electron microscopy imaging of $\mathrm{Au}(8) / \mathrm{Cr}(2) / \mathrm{Si}(001)$. (a) Bright field image of $\mathrm{Cr} / \mathrm{Si}(001)$ Schottky diode and (b) EDS imaging of $\mathrm{Au}-\mathrm{Cr}$ layers.

show distinct gold, chromium, and silicon regions with a relatively small region of intermixed chromium and silicon inset in Fig. 5.2.

The skewing of the histograms is an indication of the degree of elastic scattering of the hot electrons, which is displayed more clearly in Fig. 5.6 \& Fig. 5.7. This has been previously observed and modeled in BEEM measurements of $\mathrm{W} / \mathrm{Si}(001)$ samples [44]. The electrons that scatter elastically have their perpendicular momentum reduced, requiring a greater total energy to overcome the barrier. This increase in parallel momentum results in the electrons arriving at the interface without enough perpendicular momentum to surmount the Schottky barrier, which causes the electrons to need a greater total energy to be collected as BEEM current and skewing the distributions to higher tip biases. Another way to describe this is with a maximum or critical angle $\theta_{c}$ for transmission which is dependent upon the applied voltage $V_{t i p}$, Schottky barrier height $\phi_{B}$ and metal Fermi level $E_{f}$ that can be written as

$$
\theta_{c}\left(V_{t i p}, \phi_{B}, E_{f}\right)=\sin ^{-1} \sqrt{\frac{m_{t}}{m_{e}} \frac{V_{t i p}-\phi_{B}}{E_{f}-V_{t i p}}},
$$

where $m_{t}$ is the transverse electron effective mass in the semiconductor $\left(m_{t}=0.19 m_{e}\right.$ 

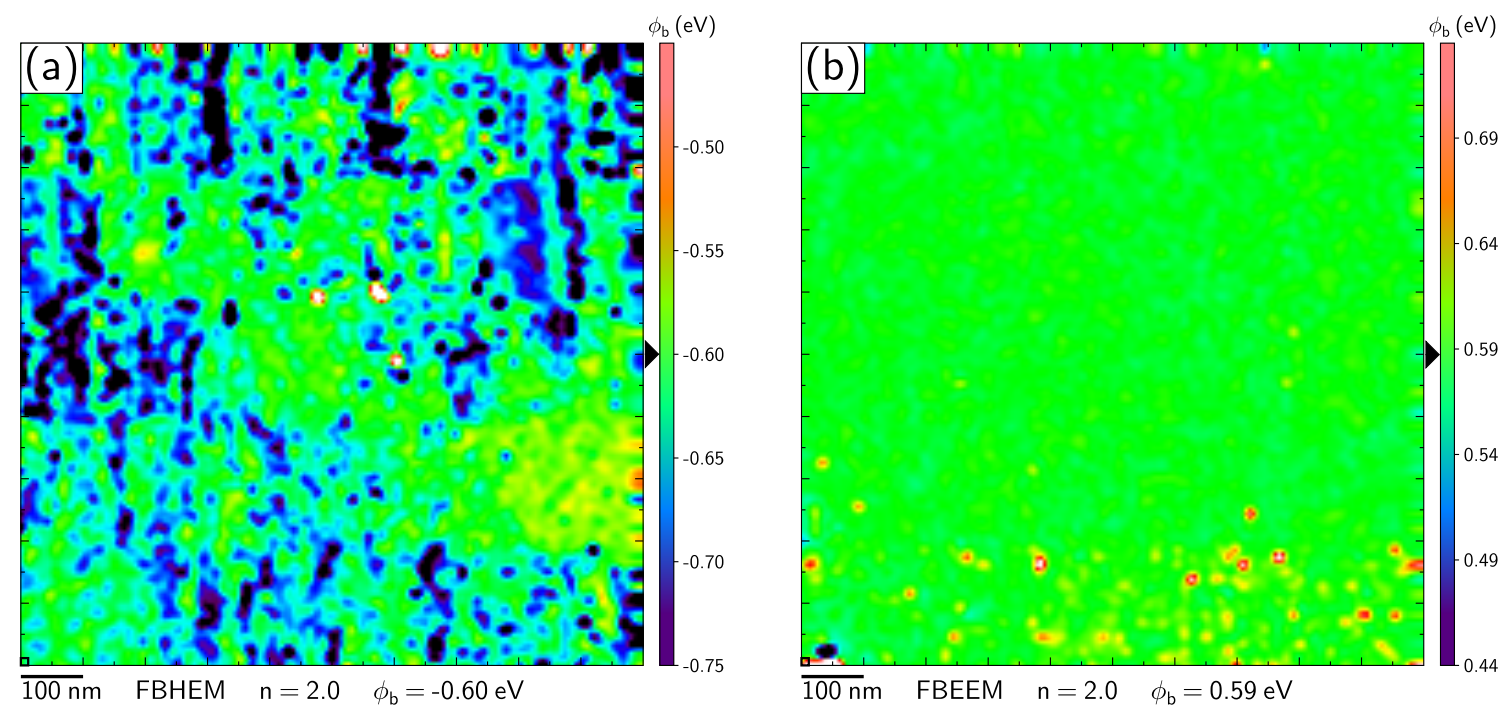

Figure 5.4: (a) $\mathrm{Cr} / \mathrm{p}-\mathrm{Si}(001)$ which shows a higher order degree of variation in the threshold values of the spectra at each tip location. (b) $\mathrm{Cr} / \mathrm{p}-\mathrm{Si}(001)$ Schottky map showing very little skewing to higher energies.

\begin{tabular}{llllll}
\hline & \multicolumn{2}{c}{ p-type } & \multicolumn{2}{c}{ n-type } \\
& Single $\phi_{t}$ & Double $\phi_{t}$ & Double $\phi_{t}$ & Single $\phi_{t}$ & Double $\phi_{t}$ \\
\hline$\phi_{t}(\mathrm{eV})$ & -0.64 & -0.64 & -0.64 & 0.59 & 0.59 \\
$\sigma$ & 0.078 & 0.078 & 0.078 & 0.023 & 0.023 \\
$\Gamma_{e}$ & $50 \%$ & $41 \%$ & $6 \%$ & $7 \%$ & $9 \%$ \\
$t(\mathrm{~nm})$ & 15 & 9 & 5 & 4 & 2 \\
$\phi_{1}(\mathrm{eV})$ & -0.61 & -0.61 & -0.60 & 0.58 & 0.58 \\
$\sigma_{1}$ & 0.051 & 0.030 & 0.030 & 0.009 & 0.009 \\
$\phi_{2}(\mathrm{eV})$ & - & -0.67 & -0.67 & - & 0.57 \\
$\sigma_{2}$ & - & 0.080 & 0.080 & - & 0.009 \\
$R(1: 2)$ & - & $60: 40$ & $60: 40$ & - & $97: 3$ \\
$\chi^{2}$ & 3.93 & 2.7 & 1.3 & 1.37 & 1.24 \\
\hline
\end{tabular}

Table 5.1: Values utilized in the modeling of the BEEM threshold distributions displayed in Fig. 5.6 \& Fig. 5.7.

in $\mathrm{Si})[23,95]$.

The results from the Monte Carlo simulation using one threshold at the interface from the mean of the data are plotted on top of the measured data and displayed in Fig. 5.6(a) \& Fig. 5.7(a). In addition, a $\chi^{2}$ value of 1.3 and 1.24 for p-type and n-type, respectively, are calculated to quantify the agreement between the model and the data. This was arrived at after an exhaustive search over all possible values of $\Gamma_{e}$, $\Gamma_{i}$, and $\sigma$. However, the quality of fit is unable to be increased by simply adjusting these parameters. The model disagrees slightly with the leading edge, peak, and tail of data for both samples. The inability to further increase the quality of fit is an indication that the skewing to higher energies and asymmetry in the distributions are 

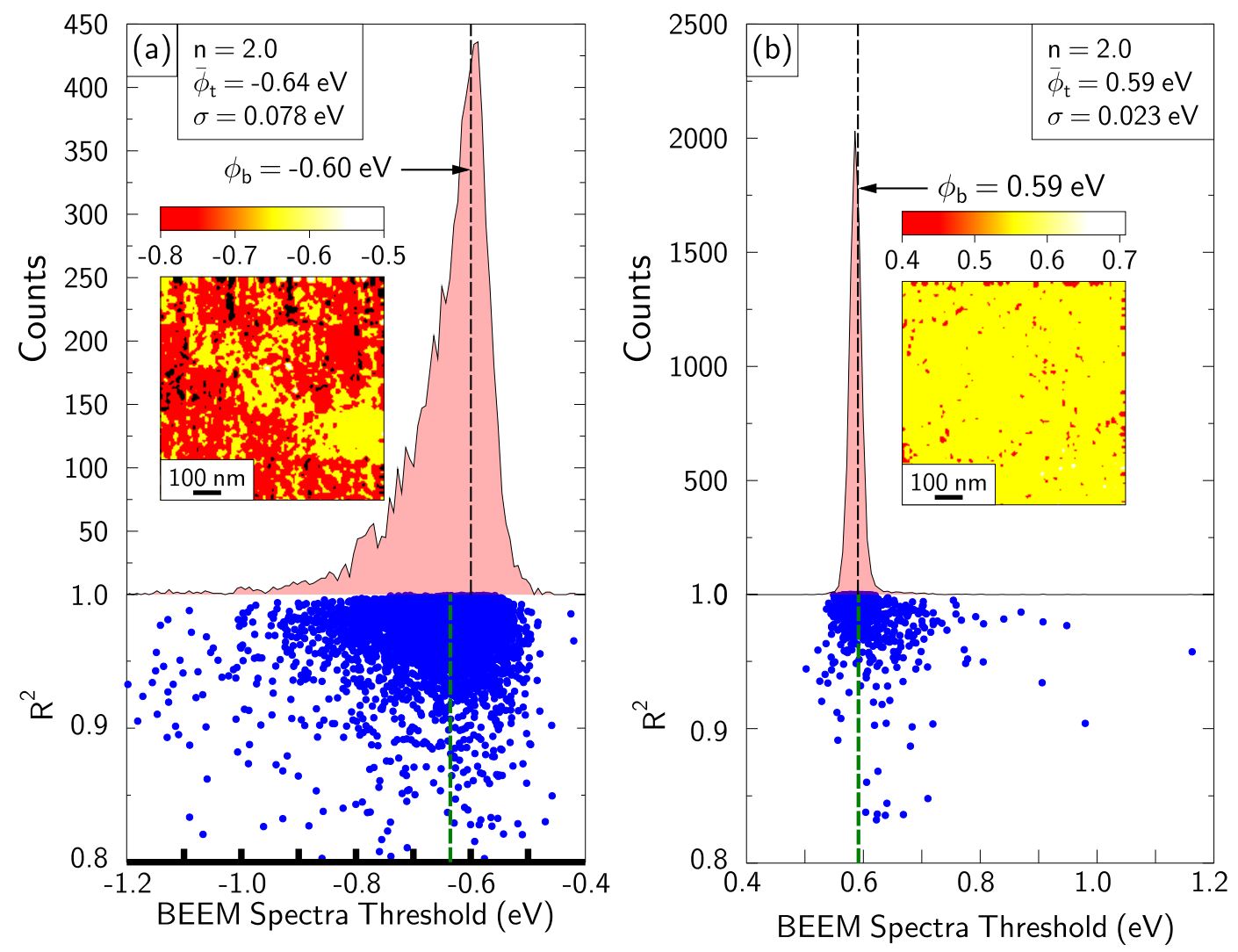

Figure 5.5: Spectra threshold distributions for (a) p-type and (b) n-type $\mathrm{Cr} / \mathrm{Si}(001)$ Schottky diode with associated $R^{2}$ values beneath. Inset is the map of the thresholds over a $1 \mu \mathrm{m}$ squared area. The lines plotted are fits to a lognormal probability distribution function.

not solely from elastic scattering.

Better agreement is achieved when including an additional threshold $\phi_{b 2}$, its standard deviation $\sigma_{2}$, and a relative threshold mixture factor $\mu$ of the amount of $\phi_{b 1}$ to $\phi_{b 2}$ at the interface in the model. The first threshold is kept fixed from the single threshold fit while a comprehensive search over $\phi_{b 2}, \sigma_{2}$ and mixture ratio $R$ is performed to maximize the $\mathrm{R}^{2}$ value. The best agreement is achieved with $\phi_{b 2}$ within a few meV of $\phi_{b 1}$ as summarized in Table. 5.1 and plotted in Fig. 5.6(b) and 5.7(b). The addition of the second threshold increases the $\chi^{2}$ values to 1.24 for $n$-type and 1.3 for p-type and provides much improved visual agreement between both distributions. $\Gamma_{e}$ was decreased in the p-type sample to achieve better agreement which brought it closer to the value used in the n-type sample as indicated in Table. 5.1. Reducing 


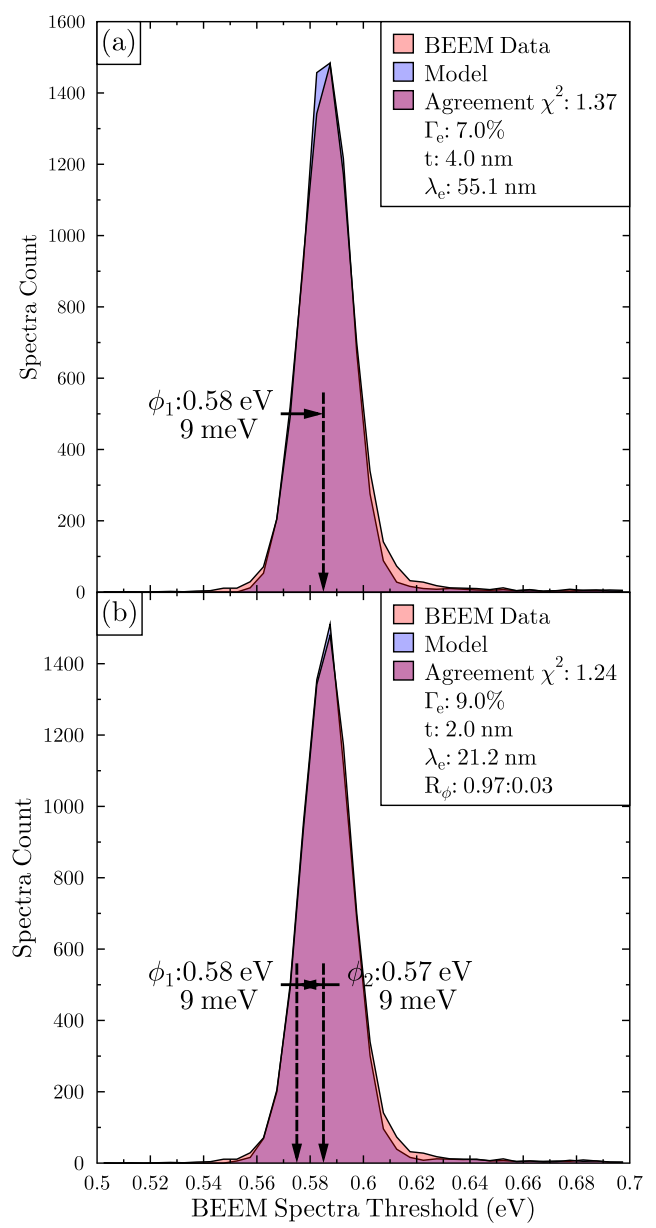

Figure 5.6: (a) $\mathrm{Cr} / \mathrm{n}-\mathrm{Si}(001)$ modelled histogram and data showing skewing to higher energies and degree of agreement between the two. (b) Improved agreement when a second threshold is added to the simulation with a mixture factor of 0.9 .

$\Gamma_{e}$ in the n-type sample did not improve agreement. The slightly larger value of $\Gamma_{e}$ for the p-type sample indicates that more elastic scattering is occurring in the p-type sample which consistent with its greater thickness.

The small differences in energy between $\phi_{b 1}$ and $\phi_{b 2}$ are $60 \mathrm{meV}$ for the ptype and $10 \mathrm{meV}$ for n-type sample and are attributed to partial chromium-silicide formation at the interface, which is supported by the slightly intermixed $\mathrm{Cr}$ and $\mathrm{Si}$ region observed in the TEM images displayed in Fig. 5.2. Typically, changes in barrier heights due to silicide formation are on the order of tens of meV as has been observed when comparing annealed and non annealed W/Si measurements [41, 44, 103, 43].

In contrast, prior Schottky barrier mapping of the miscible $\mathrm{Au}-\mathrm{Ag} / \mathrm{Si}$ diodes 


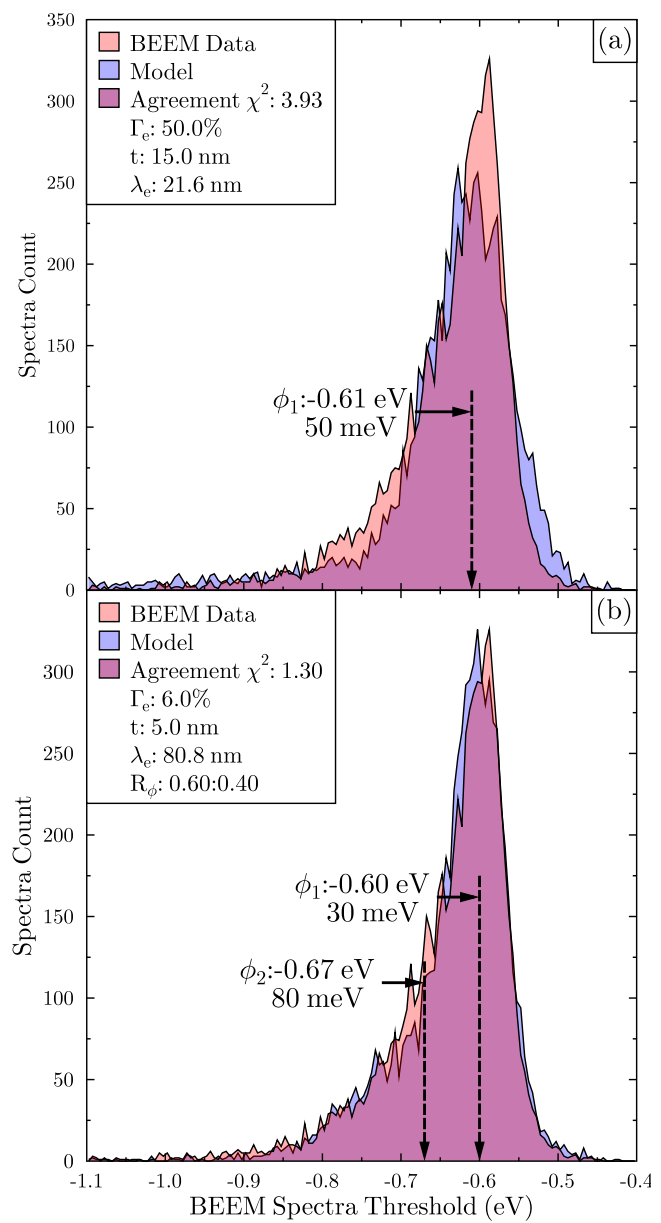

Figure 5.7: (a) $\mathrm{Cr} / \mathrm{p}-\mathrm{Si}(001)$ modelled histogram and data showing skewing to higher energies and degree of agreement between the two. (b) Improved agreement when a second threshold is added to the simulation with a mixture factor of 0.5 and a reduced $\Gamma_{e}$ of $6 \%$.

displayed much broader threshold distributions with $\sigma \approx 100 \mathrm{meV}$ and multiple peaks resulting from gold diffusion through the thin silver and the $200 \mathrm{meV}$ difference in barrier heights of the two metals to silicon [41]. Gold diffusion through these goldcapped chromium films would result in a much larger $\phi_{b 2}$ since its barrier height to silicon is $0.85 \mathrm{eV}$ (n-type) and $-0.31 \mathrm{eV}$ (p-type). In addition, gold and chromium are not as soluble with one another and do not form an alloy as readily as gold and silver, and gold is not observed at the silicon interface in the TEM results displayed in Fig. 5.2 [104, 41]. Isolated dopant atoms near the interface are neglected due to the low doping density of the substrates. The mixture factor is a measure of the relative amount of counts attributed to each barrier height at the interface. It can 

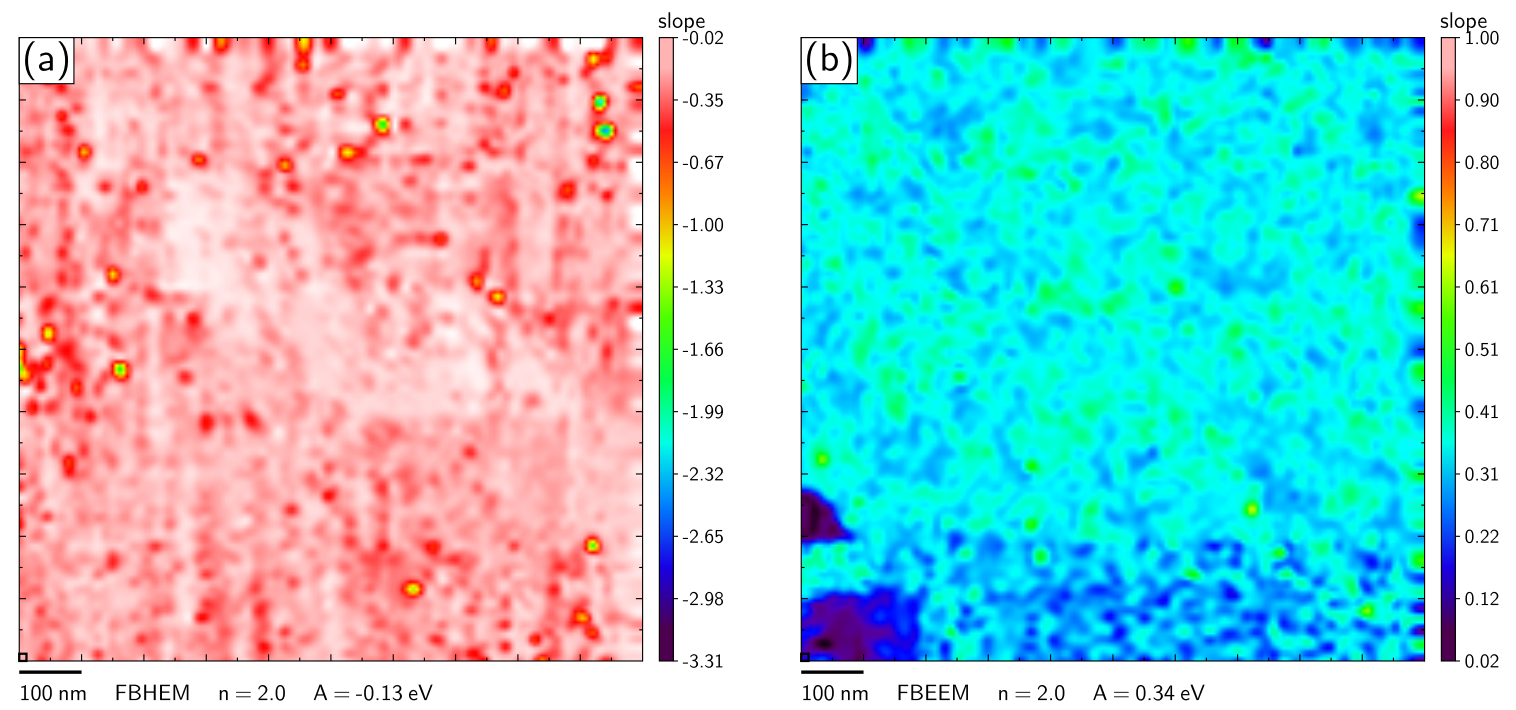

Figure 5.8: (a) $\mathrm{Cr} / \mathrm{p}-\mathrm{Si}(001)$ slope map which shows a higher order degree of variation in the slopes of the spectra at each tip location. (b) $\mathrm{Cr} / \mathrm{n}-\mathrm{Si}(001)$ slope map with a consistent value across the micron area.

be taken as a measure of the degree of silicide formation where $\mu$ for the p-type is less than the n-type. This suggests that more silicide is forming on the p-type sample as displayed in Table 5.1 and would be consistent with the thicker Cr layer of the ptype sample and possibly slight variation in processing conditions. Caution should be used when ascribing the two barrier heights reported from the modeling to a precise measure of the barrier heights for silicide and non silicide as pinch off effects tend to compress barrier heights from intermixed metal species $[6,58,40]$. This results from the continuity of the electrostatic potential at the interface and is proportional to the physical distribution of the two intermixed species.

When the BEEM spectra is fit to the simplified BEEM model, the slope of the fit is found. The slope can be an indication of thickness of the metal as well as deflection away from the interface due to elastic or inelastic scattering. Thus the slope of the barrier height is proportional to the scattering of the metal. In Fig. 5.8, the slopes of the measured chromium metal semiconductor system. In these maps we see relatively small degree of variation in the n-type $\mathrm{Si}$. The gradual increase in the slope from the top of the map to the bottom of the map could indicate a gradual increase in the thickness of the metal. In contrast, the p-type Si slope map has localized spots of increased slope and variation in the slope around those localized spots. This is 

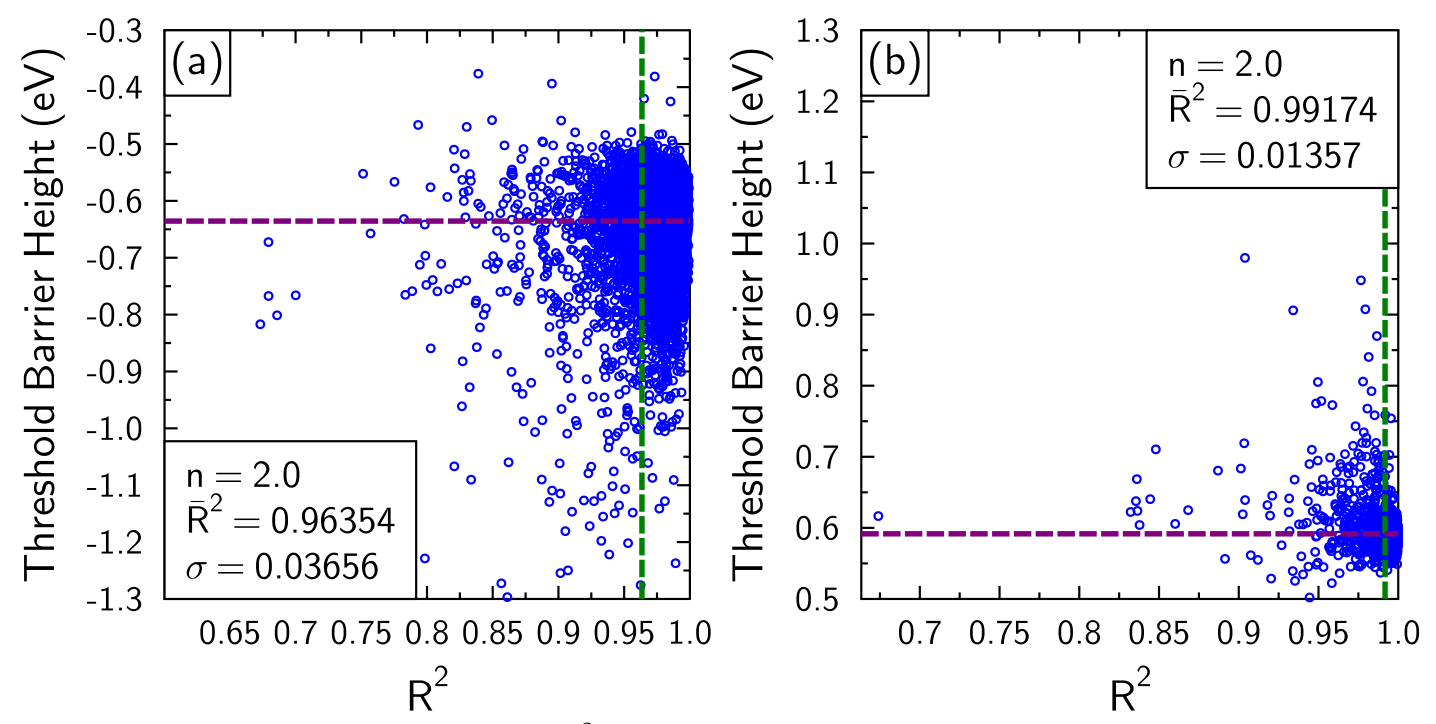

Figure 5.9: (a) Cr/p-Si(001) $R^{2}$ scatter plots of the goodness of fit and the associated threshold value which shows a higher order degree of variation in fit. (b) $\mathrm{Cr} / \mathrm{n}-\mathrm{Si}(001)$ localized to very good fitting parameters near a value of 1 .

an indication of a potential defect or scattering site that is causing deviation in the electrons path in that region. Comparing both of these slope maps to the Schottky map correlates the locations of deviation in the slope map are also locations in the Schottky map that indicates a change in the barrier height. Increasing the measured barrier height at that location.

Each fit to the BEEM spectra also produces the "goodness-of-fit" paramater, $R^{2}$. This parameter is discussed in an earlier chapter, but it indicates how well the data fits with the model. Each individual spectra is fit and the $R^{2}$ values are displayed in Fig. 5.9. These scatter plots show at a glance how well all of the data was fit over the seven to ten thousand spectra taken for a data set. These values also confirm the broadening of the histogram of threshold values and the variation in the slope accompanied by scattering encountered in the metal. These plots show that p-type Si has a broader distribution of barrier heights with accurate $\left(R^{2}>0.8\right)$ fits to each spectra.

Detection of localized silicide formation at a buried metal semiconductor interface is demonstrated via measurement of the electrostatic barrier to nanoscale dimensions and modeling with Monte-Carlo simulations. The best agreement between the model and the data is achieved when specifying two barrier heights within $60 \mathrm{meV}$ of 
one other, which provides strong evidence that localized silicide formation is occurring that would be difficult to observe in an averaged BEEM spectra or conventional current voltage measurements [45].

\subsection{Cr Thickness Study}

\subsubsection{Fermi level pinning}

Fermi-level pinning, as discussed in Chapter 2, is a prominent idea as an explanation to the formation of the Schottky barrier and prediction of the value of a given metal and semiconductor. Several studies examine the effect of the proximity of the metal atoms to the metal-semiconductor interface and the generation of metal-induced gap states (MIGS) [105, 57, 106, 107]. The work presented here aims to examine the effect of ultra-thin layers of chromium with a pinning effect generated from the gold capping layer. These ultra-thin layers are several atomic layers at their thinnest, $1.16 \mathrm{~nm}$, and many atomic layers at their thickest, $2.35 \mathrm{~nm}$ for (001). Previous studies on Fermi-level pinning reveal that MIGS can be generated by a metal layer several atomic distances away from the interface. Nishimura et al. investigated germanium with ultra-thin oxides at the interface and various metals on top of the oxide [105]. While varying the oxide thickness Nishimura was able to change the electrical properties of the diode from ohmic transport to Schottky and from Schottky to ohmic.

For this study the effects of an ultra-thin layer of $\mathrm{Cr}$ on $\mathrm{Si}(111)$ and $\mathrm{Si}(001)$ and a $\mathrm{Au}$ capping layer were examined to determine any underlying difference between choice of substrate and thickness of the transition metal. Only n-type silicon is chosen with a dopant density of $10^{-14} \mathrm{~cm}^{-3}$ to ensure that the depletion width is long for

reducing the effect of image force lowering on the barrier height [4]. Modelling of all samples was done for characterizing the effects of thickness on scattering in the metal layer.

\subsection{2 $\operatorname{Si}(100)$ Results}

$10 \mathrm{Cr}$ samples were measured with ballistic electron emission microscopy on a modified Omicron STM. All samples were measured in UHV environment and deposited with a magnetron sputter system mentioned previously. Of the 10 samples 


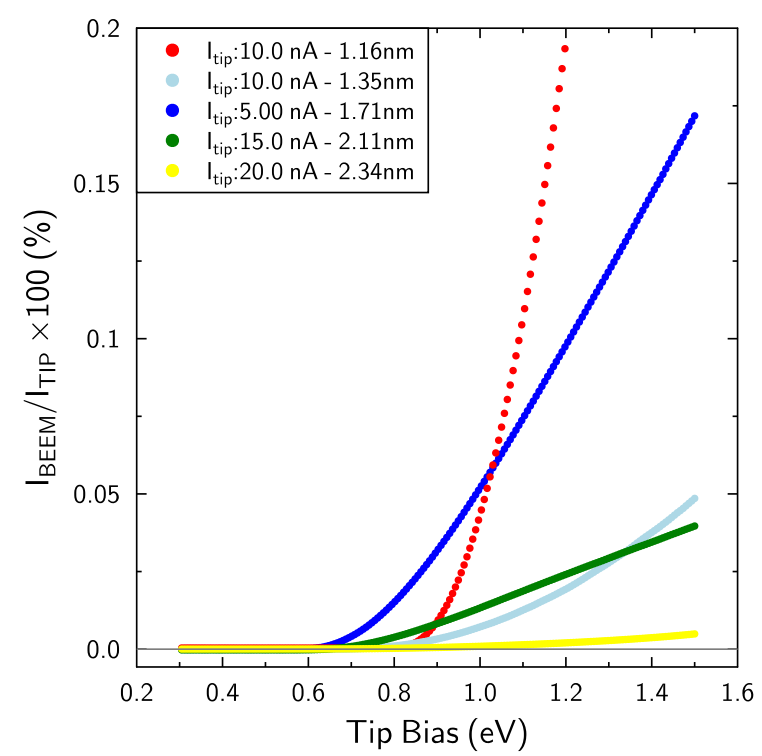

Figure 5.10: All average spectra for each of the different thicknesses are shown. An increase in $I_{t i p}$ is for increasing the percentage transmission being collected as BEEM current.

measured, 5 samples were on Si (001) with thicknesses of $1.16 \mathrm{~nm}, 1.35 \mathrm{~nm}, 1.71 \mathrm{~nm}$, $2.11 \mathrm{~nm}$ and $2.34 \mathrm{~nm}$. Thicknesses were determined by rate of deposition from magnetron sputtering with a rate of $0.12 \mathrm{~nm} / \mathrm{s}$.

Each sample was measured with BEEM at a set feedback current to ensure a measurable current can be measured on the backside contact as BEEM current. Fig. 5.10 shows the average BEEM spectra for each of the Si (100) samples. The thinnest sample at $1.16 \mathrm{~nm}$ showed the largest measured BEEM current in the spectra and was taken at $10 \mathrm{nA}$. At $1.6 \mathrm{~V}$ this is $55 \mathrm{pA}$ of BEEM current. The thickest sample at $2.34 \mathrm{~nm}$, measured at $1.6 \mathrm{~V}$ and $20 \mathrm{nA}$, showed $1 \mathrm{pA}$ of BEEM current. As thickness increases the $I_{\text {tip }}$ increases to compensate for lowered measured BEEM current.

The average BEEM spectra is shown in Fig. 5.11 for all thicknesses. All 7225 spectra are averaged together and fit to the simplified BEEM model. The thinnest sample (a) is found to have the highest barrier height at $0.83 \mathrm{eV}$. As the thickness increases the barrier Schottky barrier height decreases from $0.83 \mathrm{eV}$ to $0.59 \mathrm{eV}$ followed by a small increase at the thickest $\mathrm{Cr}$ layer to $0.61 \mathrm{eV}$. The goodness-of-fit for each of the average spectra varies between $R^{2}=0.999$ to $R^{2}=0.99999$.

A set of data for a given thickness has its histogram of BEEM spectra thresholds modelled with the computational modelling discussed in Chapter 2. The modelling 

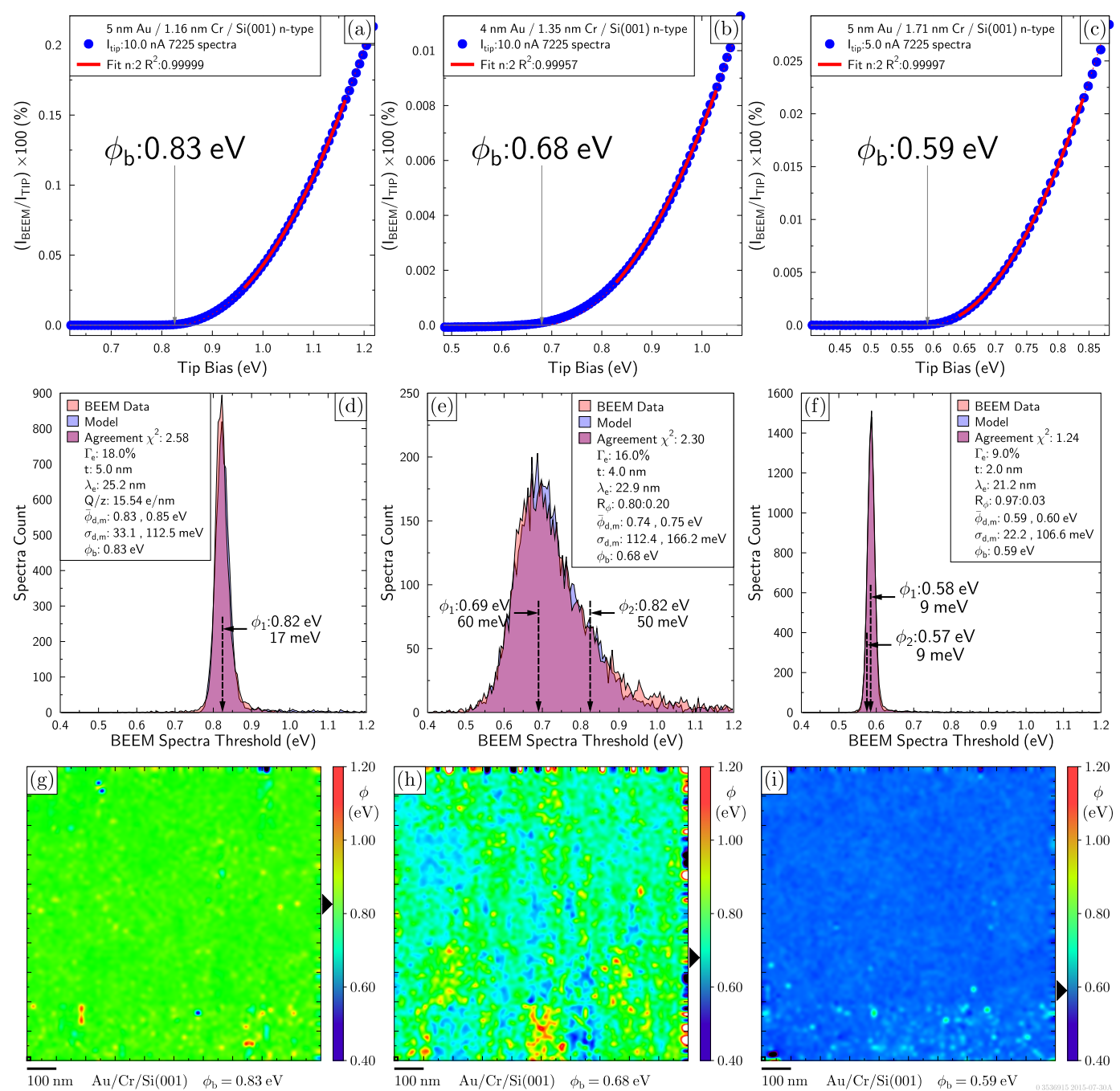

Figure 5.11: (a)(b)(c) Linear fits to the average spectra, (d)(e)(f) show histogram plots of the measured threshold values of each spectra. Each histogram gives indication of the elastic and inelastic scattering in the metal as well variation in the threshold at the interface., $(\mathrm{g})(\mathrm{h})(\mathrm{i})$ show threshold barrier height maps corresponding to thickness for chromium. (a)(d)(g) refer to $1.16 \mathrm{~nm}$ thickness, (b)(e)(h) refer to $1.35 \mathrm{~nm}$, and (c)(f)(i) refer to $1.71 \mathrm{~nm}$.

shows the number of spectra that have been skewed to higher or lower energies by elastic scattering, inelastic scattering, or fluctuation in the charge density. In Fig. 5.11, the histograms and the modelling results are shown together. How well they overlap is indicated by the $\chi^{2}$ value. For the thinnest $\mathrm{Cr}$ sample the model agrees with the data with $\chi^{2}=2.58$ and modelled barrier height of $0.82 \mathrm{eV}, \Gamma_{e}=18 \%, t=5 \mathrm{~nm}$, $Q / z=15.54 \mathrm{e} / \mathrm{nm}$ and a standard deviation of $17 \mathrm{meV}$. Each increase in thickness shows varying degrees of scattering in the metal film, with the second thinnest at 

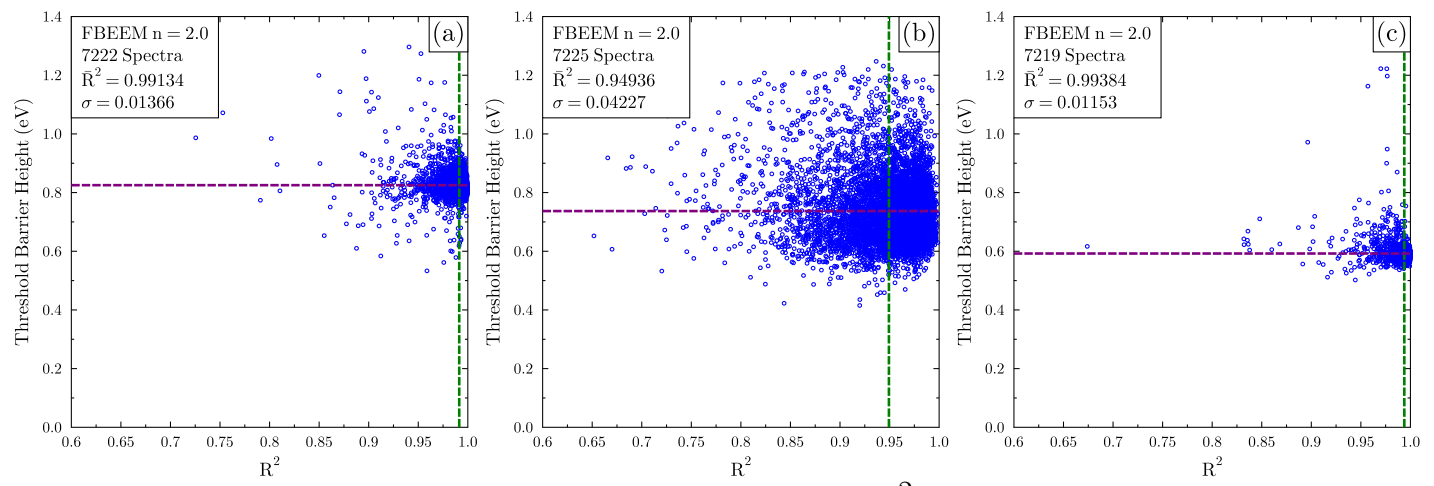

Figure 5.12: The scatter plot of the goodness of fit $R^{2}$. Tighter distributions generate more gaussian like histogram distribution of barrier heights. (a) to (c) is increasing in thickness from $1.16 \mathrm{~nm}$ to $1.35 \mathrm{~nm}$ to $1.71 \mathrm{~nm}$.

$1.35 \mathrm{~nm}$ with $\chi^{2}=3.19 \mathrm{~m}, \Gamma_{e}=28 \%, t=4 \mathrm{~nm}$, a modelled barrier height of $0.70 \mathrm{eV}$ and standard deviation of $0.081 \mathrm{meV}$.

The goodness-of-fit, $R^{2}$, is plotted in Fig. 5.12. Four of the five samples show $R^{2}$ greater than 0.94. Starting from the thinnest Cr layer the $R^{2}$ for each increase in thickness is: $R_{a}^{2}=0.99134, R_{b}^{2}=0.94936, R_{c}^{2}=0.96366, R_{d}^{2}=0.99384, R_{e}^{2}=0.97407$, and $R_{f}^{2}=0.8443$. Visual representation of the $1 \mu \mathrm{m}^{2}$ area can be represented in a threshold map of the interface. In Fig. 5.11, the Schottky barrier is shown to decrease from 0.82 to 0.6 with increasing thickness.

\subsection{3 $\quad \mathrm{Si}(111)$ Results}

$5 \mathrm{Cr} / \mathrm{Si}(111)$ samples were measured with BEEM. All samples were deposited with the magnetron sputter system mentioned previously. The samples on Si (111) were grown with thicknesses of $1.26 \mathrm{~nm}, 1.65 \mathrm{~nm}, 1.91 \mathrm{~nm}, 2.32 \mathrm{~nm}$ and $2.85 \mathrm{~nm}$. Thicknesses were determined by rate of deposition from magnetron sputtering with a rate of $0.12 \mathrm{~nm} / \mathrm{s}$.

Fig. 5.13 shows the average BEEM spectra for each of the Si-(111) samples. The thinnest sample at $1.26 \mathrm{~nm}$ shows the largest measured BEEM current in the spectra and is taken at $5 \mathrm{nA}$. At $1.0 \mathrm{~V}$ this is $5 \mathrm{pA}$ of BEEM current and the thickest sample at $2.85 \mathrm{~nm}$ measured at $1.0 \mathrm{~V}$ and $20 \mathrm{nA}$ showed less than $1 \mathrm{pA}$ of BEEM current. As with the $\operatorname{Si}(100)$ when the thickness increases the $I_{\text {tip }}$ is increased to offset for lowered transmitted BEEM current.

The average BEEM spectra is shown in Fig. 5.14 for all thicknesses. As with 


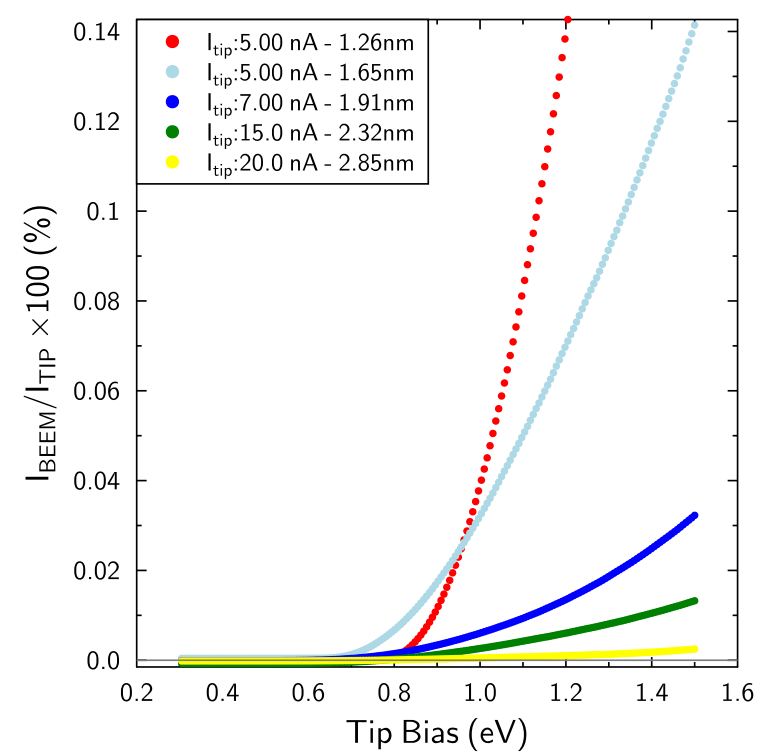

Figure 5.13: Average spectra for each of the different thicknesses are shown. An increase in $I_{t i p}$ is for increasing the percentage transmission being collected as BEEM current.

$\mathrm{Si}(100)$ samples, all 7225 spectra are averaged and it was found that the thinnest sample (a) has the highest barrier height at $0.78 \mathrm{eV}$. As the thickness increases the barrier Schottky barrier height decreases from $0.78 \mathrm{eV}$ to $0.55 \mathrm{eV}$. The goodness-of-fit for each of the average spectra varies between $R^{2}=0.999$ to $R^{2}=0.99999$.

In Fig. 5.14, the histograms and the modelling results are shown together. For the thinnest $\mathrm{Cr} / \mathrm{Si}(111)$ sample the model agrees with the data with $\chi^{2}=3.93$ an modelled barrier height of $0.79 \mathrm{eV}, \Gamma_{e}=30 \%, t=0 \mathrm{~nm}, Q / z=7.77 \mathrm{e} / \mathrm{nm}$ and a standard deviation of $25 \mathrm{meV}$. Each increase in thickness shows varying degrees of scattering in the metal film, with second thinnest at $1.35 \mathrm{~nm}$ with $\chi^{2}=3.19 \mathrm{~m}, \Gamma_{e}$ $=20 \%, t=4 \mathrm{~nm}$ and a modelled barrier height of $0.70 \mathrm{eV}$ and standard deviation of $0.081 \mathrm{meV}$. The ratio of multiple barrier heights for the (b) is $90 \%: 10 \%$. (c) in Fig. 5.14 decreases in counts while seeing an increase in elastic scattering. The trend continues with increasing thickness.

The goodness-of-fit, $R^{2}$, is plotted in Fig. 5.15. Four of five samples show $R^{2}$ greater than 0.91. Starting from the thinnest $\mathrm{Cr}$ layer the $R^{2}$ for each increase in thickness is: $R_{a}^{2}=0.96281, R_{b}^{2}=0.97410, R_{c}^{2}=0.93346, R_{d}^{2}=0.91983$, and $R_{e}^{2}=$ 0.82151. In Fig. 5.14, the Schottky barrier is shown to decrease from 0.79 to 0.55 with increasing thickness. 

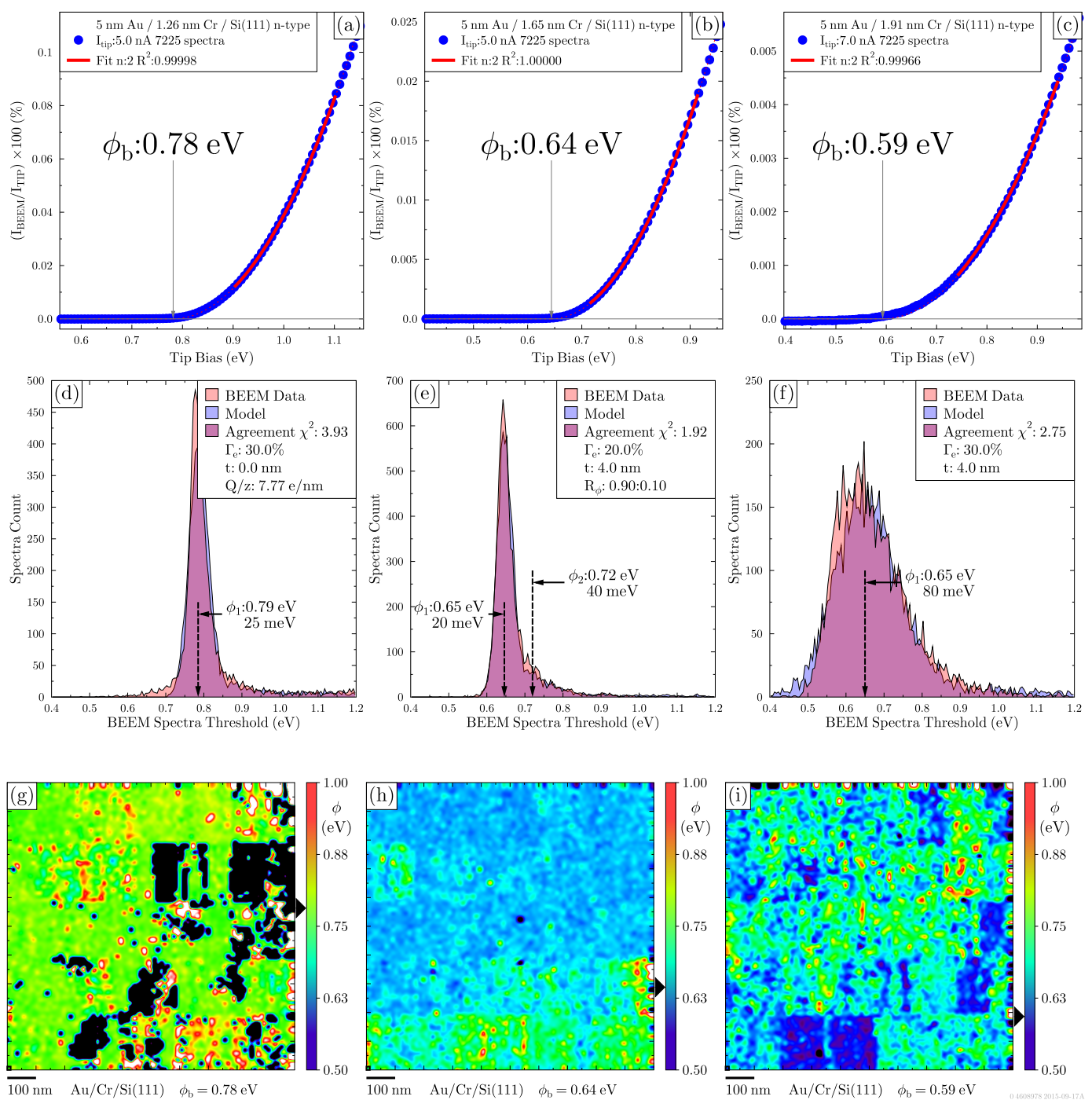

Figure 5.14: (a)(b)(c) Linear fits to the average spectra, (d)(e)(f) show histogram plots of the measured threshold values of each spectra. Each histogram gives indication of the elastic and inelastic scattering in the metal as well variation in the threshold at the interface., $(\mathrm{g})(\mathrm{h})(\mathrm{i})$ show threshold barrier height maps corresponding to thickness for chromium. (a)(d)(g) refer to $1.26 \mathrm{~nm}$ thickness, (b)(e)(h) refer to $1.65 \mathrm{~nm}$, and (c)(f)(i) refer to $1.91 \mathrm{~nm}$.

\subsubsection{Discussion}

Barrier heights presented are shown to correlate with the thickness of the $\mathrm{Cr}$ metal layer on both substrate choices, $\operatorname{Si}(111)$ and $\mathrm{Si}(100)$. The barrier heights approach the reported current-voltage measurement values of a $\mathrm{Cr}$ barrier heights on the $1.7 \mathrm{~nm}$ and $1.91 \mathrm{~nm}$ for $\mathrm{Si}(100)$ and $\mathrm{Si}(111)$ respectively. The fits to the BEEM spectra all indicate good agreement between simplified BEEM model and data. The BK 

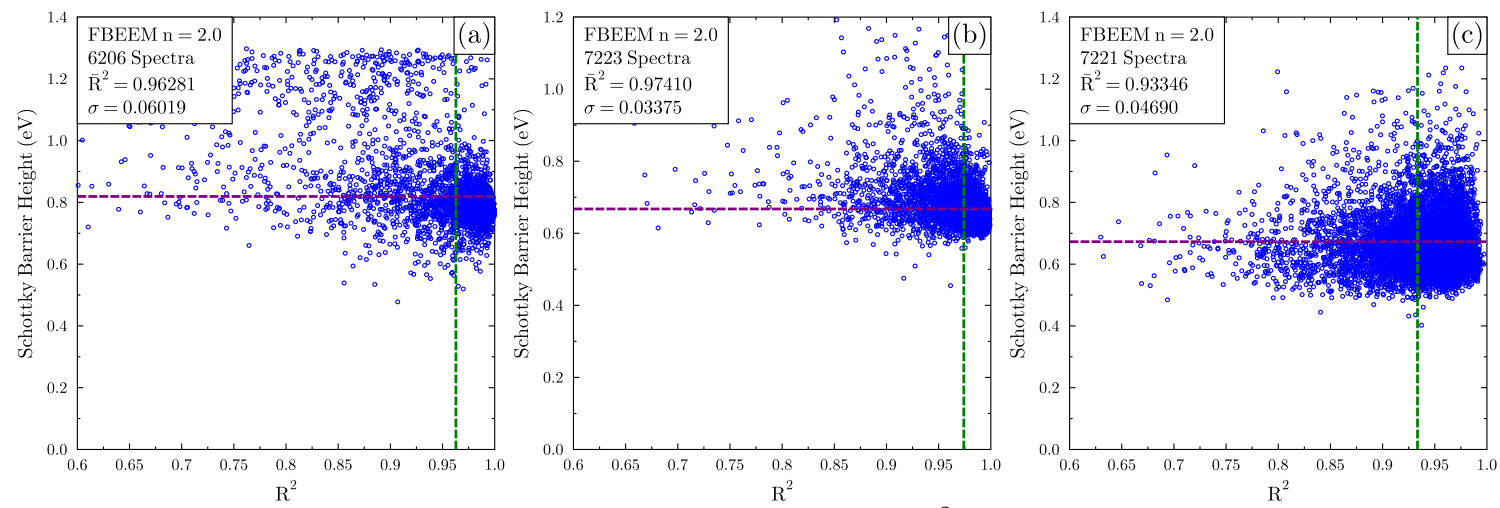

Figure 5.15: The scatter plot of the goodness of fit $R^{2}$. Tighter distributions generate more gaussian like histogram distribution of barrier heights. (a) to (c) is in increasing in thickness $1.26 \mathrm{~nm}, 1.65 \mathrm{~nm}, 1.91 \mathrm{~nm}$.

model $n=2$ is used for consistency between all reported thicknesses and in order to obtain the best goodness-of-fit. The average Schottky barrier height difference between thickest and thinnest samples also was in agreement with $\mathrm{Si}(100)$ showing $0.21 \mathrm{eV}$ change in barrier height and $\mathrm{Si}(111)$ showing a $0.23 \mathrm{eV}$ shift indicating that the overall effect of thickness is substrate independent as reports of $\mathrm{Au} / \mathrm{Si}(100)$ and $\mathrm{Au} / \mathrm{Si}(111)$ can show a shift of $0.02-0.06 \mathrm{eV}$ difference in $\phi_{B}$ between substrates $[100,108]$.

Both $\mathrm{Si}(111)$ and $\mathrm{Si}(001)$ samples were deposited in the same system and deposition environments while varying the total time of deposition. Vacuum was never breached between deposition of the two metals eliminating any possible causes for oxidation effects of the silicon or Cr layers.

Each sample is measured with 7225 spectra over $1 \mu m^{2}$ and each of these spectra are fit to the simplified BEEM model and their $R^{2}$ values are plotted vs. $\phi_{t}$. As with other data presented here, these scatter plots can help clarify underlying phenomena seen or speculated. The $R^{2}$ value confirms the accuracy of the data when compared the simplified BEEM model. The equation is able to explain aspects of the data and if the fit is not good, then the data might not be explainable by the model. Now in the case of both $\mathrm{Si}(100)$ and $\mathrm{Si}(111)$ data, the majority of the threshold values measured have an $R^{2}$ value greater than 0.9, indicating good agreement to Eq. 2.22. The thickest samples, (f) in Fig. 5.12 and (e) in Fig. 5.15, are the samples that varies greatly in $R^{2}$ which could indicate that the data taken for that sample contained a considerable degree of noise. This can be explained by the very low BEEM current seen in Fig. 5.10 and Fig. 5.13 where the thickest sample is almost completely without BEEM current 
transmission. As thickness increases for Cr with many mono-layers, the attenuation of BEEM current increases drastically due to its relatively short attenuation length and unlike other more conductive metals with longer attenuation lengths that are much greater than a monolayer $[109,99,110]$. The other $R^{2}$ scatter plots show that although the barrier height may differ by several hundred $m e V$, the goodness of fit shows that these values are accurate to the simplified BEEM model.

The modelled barrier heights and threshold histograms show a mostly increasing trend of scattering in the metal. This increasing elastic scattering is also supported by the lowering of the peak counts for each of the histograms as well as reduction of BEEM current in the average spectra. An increase in elastic scattering causes spectra to skew towards higher energies and broaden the histogram. Generally elastic scattering is caused by a few things, only one type of scattering increases from changes in thickness and reduction of BEEM current, reflection off the interface and multiple passes at overcoming the barrier height. This elastic scattering allows an electron multiple attempts at overcoming the barrier height but at a cost of requiring more energy after each pass. This has been demonstrated in Au/Si work where the change in measured BEEM current changes with quantum mechanical reflections and reduced quantum mechanical transmission probability from optical phonon scattering [111].

The decrease in measured average Schottky barrier height is an interesting phenomena since most fermi-level pinned measurements are done with oxides or dissimilar metal/si bonding [57]. These studies reveal that proximity of the metal to the interface can increase or decrease the measured barrier height and charge is only allowed to transfer between atoms at the interface of the two materials. Tung states that in reality the charge transfer can oscillate over several atomic distances. In this study, the gradual change of the barrier height is accomplished by varying a transition metal layer thickness and pinning the barrier height closer to that of $\mathrm{Au} / \mathrm{Si}$. As the $\mathrm{Cr}$ layer becomes its thinnest, the barrier height for both $\mathrm{Si}(111)$ and $\mathrm{Si}(100)$ have very good agreement. An unlike previous studies of multiple barrier heights present at the interface, the distribution of threshold values is gaussian-like and not broadened to higher energies [41]. This indicates that the measured Schottky barrier is not a combination of multiple barrier heights that can be explained by a pinch-off effect from circular patched areas of differing potentials but instead is a pinning effect bring the barrier 


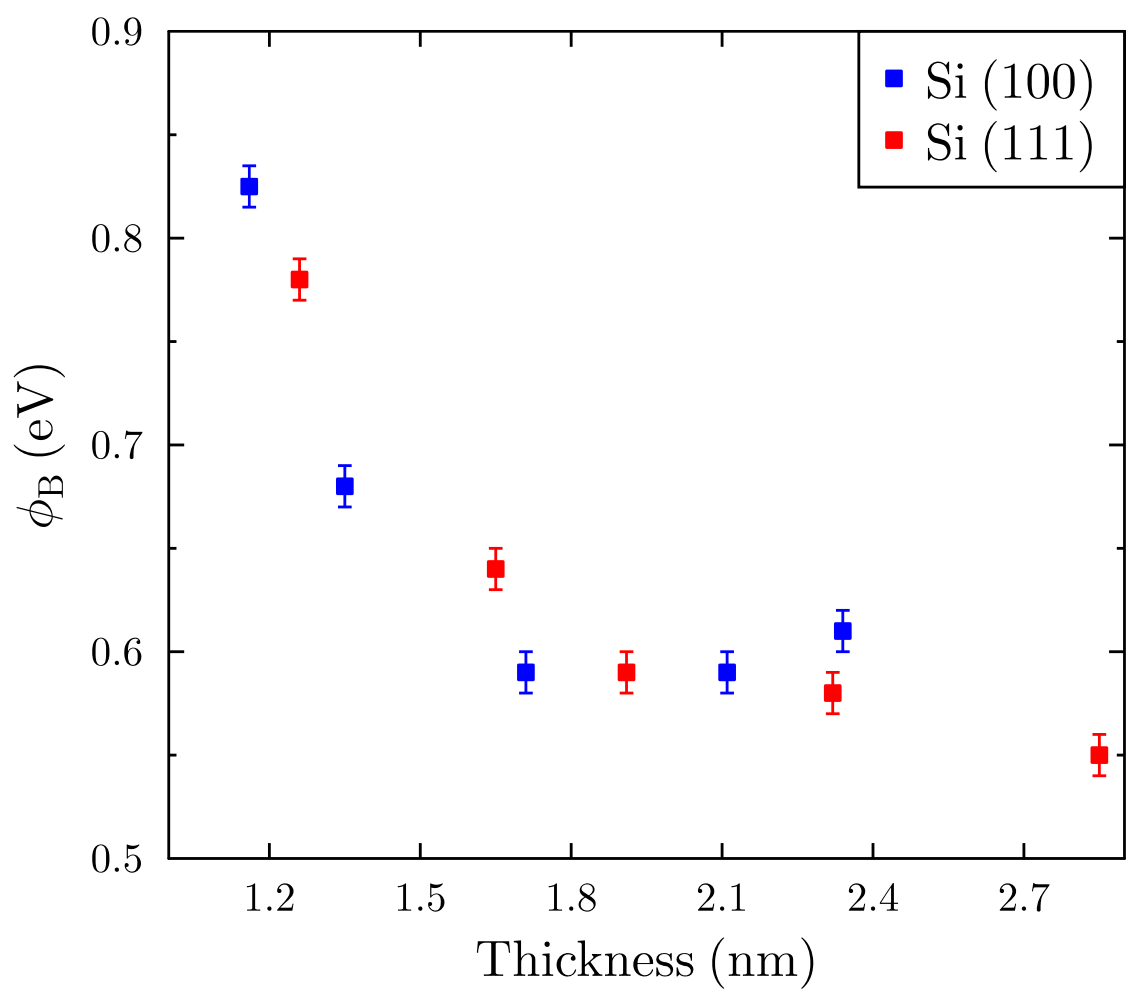

Figure 5.16: Trend of the barrier height as thickness increases. $\mathrm{Si}(100)$ and $\mathrm{Si}(111)$ both follow a similar downward slope followed by a leveling off at the barrier height of $\mathrm{Cr}$.

height somewhere between the $\mathrm{Cr}$ barrier height of $\phi_{B}=0.59 \mathrm{eV}$ and the Au barrier height of $\phi_{B}=0.85 \mathrm{eV}$.

One argument that could be made against the pinning-effect is if $\mathrm{Au}$ is intermixing with $\mathrm{Cr}$ at the interface and interdiffusion is taking place then the measured barrier height would be that of $\mathrm{Au} / \mathrm{Si}$. Franciosi et al. studied these effects on the $\mathrm{Au}-\mathrm{Cr}-\mathrm{Si}$ interface where they looked at the interdiffusion of $\mathrm{Au}$ and $\mathrm{Cr}$ on $\mathrm{Si}(111)$ [112]. In their work, the conclusions they came to were based on the number of monolayers of $\mathrm{Cr}$ at the interface. If the number of monolayers of $\mathrm{Cr}$ present at the interface was less than 10 monolayers or $0.9 \mathrm{~nm}$, then the $\mathrm{Cr}$ layer promoted interdiffusion of Si-Au to the interface and above 10 monolayers the $\mathrm{Cr}$ layer reduced the interdiffision. In the region of promotion the presence of $\mathrm{Cr}$ atoms reduces the $\mathrm{Si}-\mathrm{Si}$ bond energy and promotes sites where Au-Si bonding can be chemically driven. In contrast, once 10 monolayers is obtained, the un-reacted Cr layer at the top is able to act as a diffusion barrier to the $\mathrm{Au}$ layer.

Analysing the modelled histograms shows that there is a variation in the thick- 
ness. The $1.35 \mathrm{~nm}$ Cr sample was fit to two barriers present at the interface. In other data reported in this work, data was fit with multiple barriers were attributed to silicide formation at the interface or incomplete silicide and elastic scattering skewing thresholds to higher energies. In this Cr thickness study it is know that a silicide forms in the first 10 monolayers and analysing the difference between histograms reveals that two peaks in $1.35 \mathrm{~nm} \mathrm{Cr}$ of $\phi_{1}=0.69$ and $\phi_{2}=0.82$. Comparing this with the $1.16 \mathrm{~nm} \mathrm{Cr}$ sample shows that the $\phi_{2}$ barrier height of the $1.35 \mathrm{~nm} \mathrm{Cr}$ matches exactly to the thinnest sample. This could indicate that multiple barrier height modelling shows variations in thickness rather than just interfacial chemistry. This argument is also enhanced by examining the Schottky maps which show local areas of the interface with two different barrier heights.

In this work using BEEM the thinnest Cr layer is above the 10 monolayer limit at 13 monolayers indicating that $\mathrm{Au}-\mathrm{Cr}$ interdiffusion is an unlikely cause of shifts in the measured average Schottky barrier. The barrier height of $\mathrm{Au} / \mathrm{Si}$ is $0.85 \mathrm{eV}$ and while this is close to the thinnest $\mathrm{Cr}$ samples barrier height, for $\mathrm{Au} / \mathrm{Si}$ to show a decrease in barrier height there needs to be a underlying reason for decreasing the barrier height. A more plausible explanation is a thickness dependent effect from $\mathrm{Au}$ pinning the barrier height closer to the barrier height of $\mathrm{Au} / \mathrm{Si}$. The $1.35 \mathrm{~nm}$ sample is 2 monolayers thicker but still exhibits a pinning effect closer to the barrier height of $\mathrm{Au} / \mathrm{Si}$ which does not explain a $\mathrm{Au} / \mathrm{Si}$ barrier height present at the interface. This effect is also seen in the $\mathrm{Si}(111)$ as well. The barrier height of $\mathrm{Au} / \mathrm{Si}(111)$ is 0.89 which makes the effect even more drastic if Au was present at the interface. To make sure the Fermi-level pinning effects are accurate further investigation into the uniformity of the $\mathrm{Au} / \mathrm{Cr}$ layers is needed. 


\section{CHAPTER 6 \\ Conclusions and Future Directions}

\subsection{Conclusions}

This thesis has shown that computational modelling based on kinetic MonteCarlo methods are able to reproduce and report elastic and inelastic scattering rates, Coulomb scattering, and multiple metal species at the metal/semiconductor interface. Detection of localized silicide formation at a buried metal semiconductor interface is performed via measurement of the electrostatic barrier to nanoscale dimensions and modeling with Monte-Carlo simulation is demonstrated.

$\mathrm{W} / \mathrm{Si}$ and $\mathrm{WSi}_{2}$ depositions utilizing electron beam and RF magnetron sputter techniques to investigate the difference in barrier height measured with BEEM because of deposition technique. Results show that when depositing tungsten with electron beam evaporation unintentional substrate heating occurred during deposition which promotes growth of $\mathrm{WSi}_{2}$ layer. BEEM measurements reveal that electron scattering caused broadening of the distribution of BEEM threshold values. Modelling developed to help explain this broadening also shows that elastic scattering increases in $\mathrm{WSi}_{2}$. The best agreement between the model and the $\mathrm{W}$ data is achieved when specifying three barrier heights, two of the barrier heights within $70 \mathrm{meV}$ of one other, which provides strong evidence that localized silicide formation is occurring that would be difficult to observe in an averaged BEEM spectra or conventional current voltage measurements.

The $\mathrm{Au} / \mathrm{Ag} / \mathrm{Si}$ samples show effects of intermixed metals in contact with the semiconductor at the interface and change in barrier height because of multiple barrier heights in proximity to each other during BEEM measurements. These findings give strong evidence that the model is capable of determining the interface composition from measurements of the electrostatics with nanoscale resolution. Inferring the interface composition from the viewpoint of the electrostatics is novel and will help to improve our understanding Schottky barriers height formation as well as to understand the relationship between structural and electrostatic uniformity at the nanoscale. 
In the case of the second study outlined here, $\mathrm{Cr} / \mathrm{Si}(111)$ and $\mathrm{Cr} / \mathrm{Si}(100)$ was measured with BEEM and demonstrated a thickness dependent Schottky barrier height from proximity of the $\mathrm{Au}$ capping layer to the silicon. In a region of sub 10-monolayers of $\mathrm{Cr}$, interdiffusion of $\mathrm{Au}$ is triggered and the interface electrostatics are shown to have Au Schottky barrier values. In the samples presented here the thinnest Cr thickness of 13-monolayers is shown to have a Schottky barrier height that is close to that of $\mathrm{Au} / \mathrm{Si}$ but is lower in value and uniform in its threshold distribution. As thickness is increased the barrier height is reduced to values reported by IV for $\mathrm{Cr} / \mathrm{Si}$.

Nanoscale Schottky barrier visualization is a combination of the nanoscale maps, histograms, and modelling. Each count in the histogram has a unique position and spectra associated with it that was measured in an ordered array of tip locations and therefore should not be thought of as a global statistical histogram from a series of identically repeated measurements. In the work laid out here, nanoscale information is encoded in the histogram which enables the modelling of the nanoscale phenomenon such as barrier height distribution and location of interface charges. These statistics contain information about the hot electron transport and nanoscale fluctuations in the electrostatic barrier and is captured exceptionally well by the model. In this regard, Schottky barrier visualization as the combination of histograms, mapping, and modelling provides a new insight into the local nanoscale phenomenon of the Schottky barrier.

\subsection{Future Studies}

A sign of research being conducted well is always generating questions while answering pertinent scientific questions. The work presented in this thesis did not answer all the questions in the field of ballistic electron emission microscopy, but I believe it has generated the tools needed for pursuing new avenues that were previously closed or hard to navigate. The use of this computational modelling could give better insight into the ultra-thin layers of $\mathrm{Cr}$ on $\mathrm{Si}$ not explored here as well as thickness dependent scattering. 


\subsubsection{Ultra-thin $\mathrm{Cr} / \mathrm{Si}$}

Investigating the role of monolayers of $\mathrm{Cr}$ on the barrier height would be an excellent extension of some of the work presented here. As stated before Cr with up to 10 monolayers promotes the interdiffusion of Au to reach the Silicon. Monitoring the thickness of $\mathrm{Cr}$ as it approaches this threshold could show more dependence on metal induced gap states or would at the least provide more information on an intermixed interface of $\mathrm{Cr}-\mathrm{Au} / \mathrm{Si}$ and how the shift in barrier height reacts with multiple metals present at the interface.

This investigation would require a more in depth look at the concentrations of $\mathrm{Cr}$ and $\mathrm{Au}$ at the interface with monolayer coverage coupled with Schottky barrier measurements. X-ray photoelectron spectroscopy, TEM, EDS, and XRR measurements might explain more of what the bonding looks like at the interface and what is the role of $\mathrm{Au}$ at the interface. Also is this a $\mathrm{Au}$ dependent measurement or would other metals with barrier heights closer or further away from chromium's barrier height.

\subsubsection{Thickness dependent Scatter}

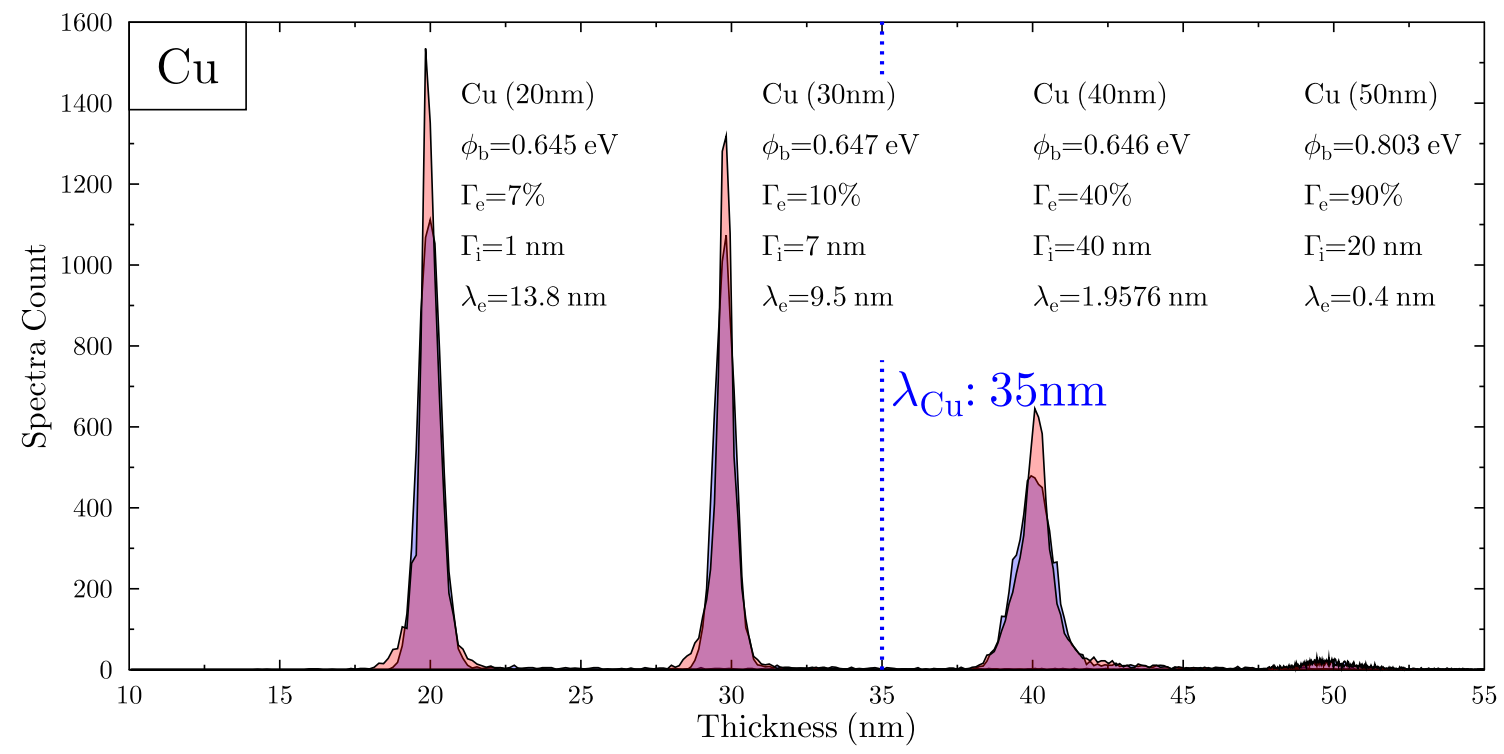

Figure 6.1: Trend of the barrier height as thickness increases. $\mathrm{Cu} / \mathrm{Si}(001)$ shows an increasing trend of scattering and attenuation of hot electrons.

Performing a thickness dependent study on various metal/semiconductor system could reveal exact nature of scattering of hot electrons during measurements. When 
BEEM data is analyzed with modelling efforts presented in this work, a moderate dependence on thickness is shown to affect the elastic and inelastic scattering rates.

In Fig. 6.1, attenuation of the BEEM signal in the threshold histograms has a significant decrease in counts after the thickness of the metal is above the attenuation length of $\mathrm{Cu}, \lambda_{C u}=35 \mathrm{~nm}$. We see that as thickness as increases the $\Gamma_{e}$ and $\Gamma_{i}$ terms also increase until most of the signal is completely attenuated well above the attenuation length of $\mathrm{Cu}$.

Thickness dependent studies in various metals would give better insight into developing modelling for predictive purposes rather than the modelling presented in this work that is more reactionary. Modelling presented in this work is done after data is collected and is used to describe the data that was collected where as a predictive model would be able to give a range of values possible or even accurately predict the exact scattering rate that the electron would encounter. 


\section{LITERATURE CITED}

[1] M. Prietsch, "Ballistic-electron emission microscopy (BEEM): studies of metal/semiconductor interfaces with nanometer resolution", Phys. Rep., 253, p. 163, (1995).

[2] S. Aftabjahani and et al., "Semiconductor Research Opportunities: An Industry Vision and Guide", Semiconductor Industry Assoc., March, p. 1, (2017).

[3] D. Edelstein, J. Heidenreich, R. Goldblatt, W. Cote, C. Uzoh, N. Lustig, P. Roper, T. McDevitt, W. Motsiff, A. Simon, J. Dukovic, R. Wachnik, H. Rathore, R. Schulz, L. Su, S. Luce, and J. Slattery, "Full copper wiring in a sub-0.25 um CMOS ULSI technology", IEDM, 10, p. 773, (1997).

[4] R. Tung, "The physics and chemistry of the Schottky barrier height", Applied Physics Reviews, 1, p. 011304, (2014).

[5] J. M. Mooney, "The dependence of the Schottky emission coefficient on reverse bias", J. Appl. Phys., 65, p. 2869, (1989).

[6] R. T. Tung, "Electron transport at metal-semiconductor interfaces: General theory", Phys. Rev. B, 45, p. 13509, (1992).

[7] J. Tersoff, "Schottky barriers and semiconductor band structures", Phys. Rev. B, 32, p. 6968, (1985).

[8] D. L. Carroll, M. Wagner, M. Ruhle, and D. A. Bonnell, "Schottky-barrier formation at nanoscale metal-oxide interfaces", Phys. Rev. B, 55, p. 9792, (1997).

[9] J. Y. Duboz, P. A. Badoz, F. Arnaud d'Avitaya, and E. Rosencher, "Evidence for Fermi-energy pinning relative to either valence or conduction band in Schottky barriers", Phys. Rev. B, 40, p. 10607, (1989).

[10] Y. Jiao, A. Hellman, Y. Fang, S. Gao, and M. Kll1, "Schottky barrier formation and band bending revealed by first- principles calculations", srep, 5, p. 11374, (2015).

[11] L. D. Bell and W. J. Kaiser, "Observation of interface band structure by ballistic-electron-emission microscopy", Phys. Rev. Lett., 61, p. 2368, (1988).

[12] F. Braun, "On current conduction through metal sulfides", Ann. Phys. -Leipzig, 153, p. 556, (1875). 
[13] G. W. Pierce, "Crystal Rectifiers for Electric Currents and Electric Oscillations. Part I. Carborundum", Phys. Rev., 25, p. 31, (1907).

[14] C.-T. Sah, "Fundamentals of Solid State Electronics" (World Scientific Pub Co Inc, Singapore, 1991).

[15] M. A. Shampo, R. A. Kyle, and D. P. Steensma, "William Shockley and the Transistor", Mayo Clinic Proceedings, 87, p. e43, (2012).

[16] N. Mott, "The Theory of Crystal Rectifiers", Proc. R. Soc., 171, p. 27, (1939).

[17] W. Schottky, "Semiconductor theory of the junction and peak rectifier", Z. Phys., 113, p. 367, (1939).

[18] W. Schottky and E. Spenke, "Quantitative treatment of the space charge and boundary-layer theory of the crystal rectifier", Wiss. Veroff. Siemens-Werken, (1939).

[19] H. Bethe, "Theory of high frequency rectification by silicon crystals.", MIT Radiation Lab Tech. Rep., 43-11, p. 12, (1942).

[20] H. Bethe, "Theory of the boundary layer of crystal rectifiers", MIT Radiation Lab Tech. Rep., 43-12, p. 23, (1942).

[21] G. Binning, H. Rohrer, C. Gerber, and W. Weibel, "Surface studies by scanning tunneling microscopy", Phys. Rev. Lett., 49, p. 57, (1982).

[22] G. Binnig, H. Rohrer, C. Gerber, and E. Weibel, "Tunneling through a controllable vacuum gap", Appl. Phys. Lett., 40, p. 178, (1982).

[23] W. J. Kaiser and L. D. Bell, "Direct investigation of subsurface interface electronic structure by ballistic-electron-emission microscopy", Phys. Rev. Lett., 60, p. 1406, (1988).

[24] R. Ludeke and M. Prietsch, "Ballistic electron emission spectroscopy of noble metal-GaP(110) interfaces", J. Vac. Sci. Technol. A, 9, p. 885, (1991).

[25] L. J. Brillson, "The structure and properties of metal-semiconductor interfaces", Surf. Sci. Rep., 2, p. 123, (1982).

[26] W. E. Spicer, I. Lindau, P. Skeath, C. Y. Su, and P. Chye, "Unified Mechanism for Schottky-Barrier Formation and III-V Oxide Interface States", Phys. Rev. Lett., 44, p. 420, (1980).

[27] P. W. Chye, I. Lindau, P. Pianetta, C. M. Garner, C. Y. Su, and W. E. Spicer, "Photoemission study of Au Schottky-barrier formation on GaSb, GaAs, and InP using synchrotron radiation", Phys. Rev. B, 18, p. 5545, (1978). 
[28] L. J. Schowalter and E. Y. Lee, "Role of elastic scattering in ballistic-electron-emission microscopy of $\mathrm{Au} / \mathrm{Si}(001)$ and Au/Si(111) interfaces", Phys. Rev. B, 43, p. 9308, (1991).

[29] A. Fernandez, H. D. Hallen, T. Huang, R. A. Buhrman, and J. Silcox, "Elastic scattering in ballistic-electron-emission-microscopy studies of the epitaxial $\mathrm{NiSi}_{2} / \mathrm{Si}(111)$ interface", Phys. Rev. B, 44, p. 3428, (1991).

[30] F. J. Garcia-Vidal, P. L. de Andres, and F. Flores, "Elastic scattering and the lateral resolution of ballistic electron emission microscopy: focusing effects on the Au/Si interface", Phys. Rev. Lett., 76, p. 807, (1996).

[31] A. M. Milliken, S. J. Manion, W. J. Kaiser, L. D. Bell, and M. H. Hecht, "Probing hot-carrier transport and elastic scattering using ballistic-electron-emission microscopy", Phys. Rev. B, 46, p. 12826, (1992).

[32] P. Niedermann, L. Quattropani, K. Solt, I. Maggio-Aprile, and O. Fischer, "Hot-carrier scattering in a metal: a ballistic-electron-emission microscopy investigation on PtSi", Phys. Rev. B, 48, p. 8833, (1993).

[33] L. D. Bell, W. J. Kaiser, M. H. Hecht, and L. C. Davis, "New electron and hole spectroscopies based on ballistic electron emission microscopy", J. Vac. Sci. Technol. B, 9, p. 594, (1991).

[34] C. Crowell and S. Sze, "Physics of Thin Films" (Academic Press, New York, 1967).

[35] T. Sumiya, T. Miura, H. Fujinuma, and S. Tanaka, "Schottky barrier inhomogeneity at $\mathrm{Au} / \mathrm{Si}(111)$ interfaces investigated using ultrahigh-vacuum ballistic electron emission microscopy", Appl. Surf. Sci., 117-118, p. 329, (1997).

[36] H. Palm, M. Arbes, and M. Schulz, "Fluctuations of the Au-Si(100) Schottky Barrier Height", Phys. Rev. Lett., 71, p. 2224, (1993).

[37] H. Palm, M. Arbes, and M. Schulz, "Nanometer-microscopy of the electron transmission through an ultrathin $(322 \mathrm{~nm}) \mathrm{Au}$ film and of the Au-Si schottky barrier height", Appl. Phys. A, 56, p. 1, (1993).

[38] K. E. J. Goh, A. Bannani1, and C. Troadec, "Imaging buried organic islands byspatially resolved ballistic electronemission spectroscopy", Nanotechnology, 19, p. 445718, (2008).

[39] A. Olbrich, J. Vancea, F. Kreupl, and H. Hoffmann, "The origin of the integral barrier height in inhomogeneous 
$\mathrm{Au} / \mathrm{Co} / \mathrm{GaAs}_{67} \mathrm{P}_{33}$-Schottky contacts: a ballistic electron emission microscopy study", J. Appl. Phys., 83, p. 358, (1998).

[40] A. Olbrich, J. Vancea, F. Kreupl, and H. Hoffmann, "Potential pinch-off effect in inhomogeneous $\mathrm{Au} / \mathrm{Co} / \mathrm{GaAs}_{67} \mathrm{P}_{33}(100)$ - Schottky contacts", Appl. Phys. Lett., 70, p. 2559, (1997).

[41] R. Balsano, C. Durcan, A. Matsubayashi, A. J. Narasimham, and V. P. LaBella, "Relating Spatially Resolved Maps of the Schottky Barrier Height to Metal/Semiconductor Interface Composition", J. Appl. Phys., 119, p. 095302, (2016).

[42] C. A. Durcan, R. Balsano, and V. P. LaBella, "Nanoscale Mapping of the W/Si(001) Schottky Barrier", J. Appl. Phys., 116, p. 023705, (2014).

[43] C. A. Durcan, R. Balsano, and V. P. LaBella, "Time dependent changes in Schottky barrier mapping of the $\mathrm{W} / \mathrm{Si}(001)$ interface utilizing Ballistic Electron Emission Microscopy", J. Appl. Phys., 117, p. 245306, (2015).

[44] W. Nolting, C. Durcan, A. J. Narasimham, and V. P. LaBella, "Nanoscale Schottky barrier mapping of thermally evaporated and sputter deposited $\mathrm{W} / \mathrm{Si}(001)$ diodes using ballistic electron emission microscopy", J. Vac. Sci. Technol. B, 34, p. 04J110, (2016).

[45] W. Nolting, C. Durcan, and V. P. LaBella, "Detection of Silicide Formation in Nanoscale Visualization of Interface Electrostatics", Appl. Phys. Lett., 110, p. 141606, (2017).

[46] G. Binnig and H. Rohrer, "Scanning tunneling microscopy", IBM J. Res. Dev., 30, p. 355, (1986).

[47] C. Chen, "Introduction to Scanning Tunneling Microscopy" (Oxford University Press, New York, 2008).

[48] D. J. Griffiths, "Introduction to Quantum Mechanics" (Prentice Hall, Upper Saddle River, New Jersey, 1995).

[49] E. Merzbacher, "Quantum Mechanics" (John Wiley \& Sons Inc., 1962).

[50] J. P. Sullivan, R. T. Tung, M. R. Pinto, and W. R. Graham, "Electron transport of inhomogeneous Schottky barriers: A numerical study", J. Appl. Phys., 70, p. 7403, (1991).

[51] A. A. Talin, D. A. A. Ohlberg, R. S. Williams, P. Sullivan, loannis Koutselas, B. Williams, and K. L. Kavanagh, "Time dependent ballistic electron emission microscopy studies of a $\mathrm{Au} /(\mathrm{IOO}) \mathrm{GaAs}$ interface with a native oxide diffusion barrier", Appl. Phys. Lett., 62, p. 2965, (1993). 
[52] T. dell'Orto, G. De Stasio, M. Capozi, C. Ottaviani, C. Quaresima, and P. Perfetti, "Band-offset formation in the a-Si/Si(111) homojunction by a CaF $_{2}$ intralayer", Phys. Rev. B, 48, p. 8823, (1993).

[53] P.E.Schmid, "Silicide-Silicon Schottky Barriers", Acta. Cryst. B, 58, p. $371,(1985)$.

[54] A. P. Horsfield, S. D. Kenny, and H. Fujitani, "Density-functional study of adsorption of Co on Si(100)", Phys. Rev. B, 64, p. 245332, (2001).

[55] R. T. Tung, "Schottky barrier heights of single crystal silicides on Si(111)", J. Vac. Sci. Technol. B, 2, p. 465, (1984).

[56] A. M. Cowley and S. M. Sze, "Surface States and Barrier Height of Metal-Semiconductor Systems", J. Appl. Phys., 36, p. 3212, (1965).

[57] R. T. Tung, "Chemical Bonding and Fermi Level Pinning at Metal-Semiconductor Interfaces", Phys. Rev. Lett., 84, p. 6078, (2000).

[58] R. T. Tung, "Recent advances in Schottky barrier concepts", Mater. Sci. Eng. R Rep., 35, p. 1, (2001).

[59] S. G. Louie and M. L. Cohen, "Self-Consistent Pseudopotential Calculation for a Metal-Semiconductor Interface", Phys. Rev. Lett., 35, p. 866, (1975).

[60] J. M. Andrews and J. C. Phillips, "Chemical Bonding and Structure of Metal-Semiconductor Interfaces", Phys. Rev. Lett., 35, p. 56, (1975).

[61] P. Esslinger and K. Schubert, "Zur systematik der strukturfamilie des NiAs. 1. Verbreitung der strukturen der NiAs-familie", J. Mater. Res. (USA), 48, p. 126, (1957).

[62] Y. Jiao, A. Hellman, Y. Fang, S. Gao, and M. Kll, "Schottky barrier formation and band bending revealed by first- principles calculations", Sci. Rep., 5, p. 11374, (2015).

[63] M. Prietsch and R. Ludeke, "BEEM spectroscopy at interfaces of Au, $\mathrm{Ag}, \mathrm{Cu}, \mathrm{Mg}$ and Ni films with n-GaP(110)", Surf. Sci., 251-252, p. 413, (1991).

[64] J. S. Chawla, F. Gstrein, K. P. OBrien, J. S. Clarke, and D. Gall, "Electron scattering at surfaces and grain boundaries in $\mathrm{Cu}$ thin films and wires", Phys. Rev. B, 84, p. 235423, (2011).

[65] G. Kastle, H. G. Boyen, A. Schroder, A. Plettl, and P. Ziemann, "Size effect of the resistivity of thin epitaxial gold films test", Phys. Rev. B, 70, p. 165414, (2004). 
[66] A. F. Mayadas and M. Shatzkes, "Electrical-Resistivity Model for Polycrystalline Films: the Case of Arbitrary Re6ection at External Surfaces", Phys. Rev. B, 1, p. 1382, (1970).

[67] W. Zhang, S. H. Brongersma, O. Richard, B. Brijs, R. Palmans, L. Froyen, and K. Maex, "Influence of the electron mean free path on the resistivity of thin metal films", Microelectron. Eng., 76, p. 146, (2004).

[68] G. N. Gould and L. A. Moraga, "A method for fitting the Fuchs-Sondheimer theory to resistivity-thickness measurements for all film thicknesses", Thin Solid Films, 10, p. 327, (1972).

[69] E. Sondheimer, "The mean free path of electrons in metals", $A d v$. Phys., 1, p. 1, (1952).

[70] K. Fuchs, "The conductivity of thin metallic films according to the electron theory of metals", Proc Camb Phil Soc, 34, p. 100, (1938).

[71] J. J. Garramone, J. R. Abel, S. Barraza-Lopez, and V. P. LaBella, "Signatures of the semiconductor crystallographic orientation on the charge transport across non-epitaxial diodes", Appl. Phys. Lett., 100, p. 252102, (2012).

[72] A. Matthiessen and C. Vogt, "On the influence of temperature on the electric conducting-power of alloys", Philos. Trans. R. Soc. Lond. A, Math. Phys. Sci. (UK), 154, p. 167, (1864).

[73] E. Zarate, P. Apell, and P. M. Echenique, "Calculation of low-energy-electron lifetimes", Phys. Rev. B, 60, p. 2326, (1999).

[74] G. W. Snedecor and W. G. Cochran, "Statistical Methods" (Iowa State University Press., 1989).

[75] W. H. Press, S. A. Teukolsky, W. T. Vetterling, and B. P. Flannery, "NUMERICAL RECIPES IN C: THE ART OF SCIENTIFIC COMPUTING" (Cambridge University Press, 1988).

[76] BOOST C++ Libraries. http://www.boost.org/ 2018-05-09.

[77] D. Graf, M. Grundner, and R. Schulz, "Reaction of water with hydrofluoric acid treated silicon(111) and (100) surfaces", J. Vac. Sci. Technol. A, 7, p. 808, (1989).

[78] A. Bannani, C. A. Bobisch, M. Matena, and R. Moller, "Ballistic electron emission spectroscopy on Ag/Si devices", Nanotechnology, 19, p. 375706, (2008).

[79] R. F. Schmitsdorf, T. U. Kampen, and W. Monch, "Correlation between barrier height and interface structure of $\mathrm{Ag} / \mathrm{Si}(111)$ Schottky diodes", Surf. Sci., 324, p. 249, (1995). 
[80] M. Morita, T. Ohmi, E. Hasegawa, M. Kawakami, and M. Ohwada, "Growth of native oxide on a silicon surface", J. Appl. Phys., 68, p. 1272, (1990).

[81] B. E. Deal and A. S. Grove, "General Relationship for the Thermal Oxidation of Silicon", J. Appl. Phys., 36, p. 3770, (1965).

[82] K. R. Williams and R. S. Muller, "Etch Rates for Micromachining Processing", jmems, 5, p. 96, (1996).

[83] G. S. Higashi, R. S. Becker, Y. J. Chabal, and A. J. Becker, "Comparison of $\mathrm{Si}(111)$ surfaces prepared using aqueous solutions of $\mathrm{NH} 4 \mathrm{~F}$ versus HF", Appl. Phys. Lett., 58, p. 1656, (1991).

[84] K. S. Harsha, "Principles of Vapor Deposition of Thin Films" (Elsevier Science, Great Britain, 2005).

[85] M. Krause, A. Stollenwerk, C. Awo-Affouda, B. Maclean, and V. P. LaBella, "Combined molecular beam epitaxy low temperature scanning tunneling microscopy system: enabling atomic scale characterization of semiconductor surfaces and interfaces", J. Vac. Sci. Technol. B, 23, p. 1684, (2005).

[86] M. Khaidar, A. Essafti, A. Bennouna, E. L. Ameziane, and M. Brunel, "rf sputtered tungsten amorphous silicon Schottky barrier diodes", J. Appl. Phys., 65, p. 3248, (1989).

[87] M. Aboelfotoh, "Electrical characteristics of WSi(100) Schottky barrier junctions", J. Appl. Phys., 66, p. 262, (1989).

[88] M. Mamor, F. D. Auret, S. A. Goodman, and G. Myburg, "Electrical characterization of defects introduced in $\mathrm{p}-\mathrm{Si}_{1-x} \mathrm{Ge}_{x}$ during electron-beam deposition of Sc Schottky barrier diodes", Appl. Phys. Lett., 72, p. 1069, (1998).

[89] W. Bludau, A. Onton, and W. Heinke, "Temperature dependence of the band gap of silicon", J. Appl. Phys., 45, p. 1846, (1974).

[90] S. M. Sze, "Physics of Semiconductor Devices" (John Wiley \& Sons, Inc., New York, 1981).

[91] R. Balsano, A. Matsubayashi, and V. P. LaBella, "Schottky barrier height measurements of $\mathrm{Cu} / \mathrm{Si}(001), \mathrm{Ag} / \mathrm{Si}(001)$, and $\mathrm{Au} / \mathrm{Si}(001)$ interfaces utilizing ballistic electron emission microscopy and ballistic hole emission microscopy", AIP Advances, 3, p. 112110, (2013).

[92] F. D. Auret and P. M. Mooney, "Deep levels introduced during electron-beam deposition of metals on n-type silicon", J. Appl. Phys., 55, p. 988, (1984). 
[93] F. D. Auret and P. M. Mooney, "Transient capacitance study of defects introduced by electronbeam deposition of metals on ptype silicon", J. Appl. Phys., 55, p. 984, (1984).

[94] H. J. Im, Y. Ding, J. P. Pelz, and W. J. Choyke, "Nanometer-scale test of the Tung model of Schottky-barrier height inhomogeneity", Phys. Rev. B, 64, p. 075310, (2001).

[95] L. D. Bell, "Evidence of momentum conservation at a nonepitaxial metal/semiconductor interface using ballistic electron emission microscopy", Phys. Rev. Lett., 77, p. 3893, (1996).

[96] T. Arizumi, M. Hirose, and N. Altaf, "Au-Ag Alloy-Silicon Schottky Barriers", Jpn. J. Appl. Phys., 7, p. 870, (1968).

[97] J. J. Garramone, J. R. Abel, I. L. Sitnitsky, and V. P. LaBella, "Hot-Electron Transport Studies of the $\mathrm{Ag} / \mathrm{Si}(001)$ Interface Using Ballistic Electron Emission Microscopy", J. Vac. Sci. Technol. A, 28, p. $643,(2010)$.

[98] C. A. Ventrice, Jr., V. P. LaBella, G. Ramaswamy, H. P. Yu, and L. J. Schowalter, "Measurement of hot-electron scattering processes at $\mathrm{Au} / \mathrm{Si}(100)$ Schottky interfaces by temperature-dependent ballistic-electron-emission microscopy", Phys. Rev. B, 53, p. 3952, (1996).

[99] J. J. Garramone, J. R. Abel, I. L. Sitnitsky, L. Zhao, I. Appelbaum, and V. P. LaBella, "Measurement of the Hot Electron Attenuation Length of Copper", Appl. Phys. Lett., 96, p. 062105, (2010).

[100] M. K. Weilmeier, W. H. Rippard, and R. A. Buhrman, "Ballistic electron transport through $\mathrm{Au}(111) / \mathrm{Si}(111)$ and $\mathrm{Au}(111) / \mathrm{Si}(100)$ interfaces", Phys. Rev. B, 59, p. R2521, (1999).

[101] C. Feautrier1, A. S. Ozcan, C. Lavoie, A. Valery, R. Beneyton, C. Borowiak, L. Clment, A. Pofelski, , and B. Salem, "Impact of laser anneal on NiPt silicide texture and chemical composition", J. Appl. Phys., 121, p. 225109, (2017).

[102] C. Lavoie, F. dHeurle, C. Detavernier, and C. C. Jr., "Towards implementation of a nickel silicide process for CMOS technologies", Microelectron. Eng., 70, p. 144, (2003).

[103] P. M. Gammon, A. Prez-Toms, V. A. Shah, O. Vavasour, E. Donchev, J. S. Pang, M. Myronov, C. A. Fisher, M. R. Jennings, D. R. Leadley, , and P. A. Mawby, "Modelling the inhomogeneous SiC Schottky interface", $J$. Appl. Phys., 114, p. 223704, (2013). 
[104] G. Gottstein, "Physical Foundations of Materials Science" (Springer-Verlag, Berlin, 2004).

[105] T. Nishimura, K. Kita, and A. Toriumi, "A Significant Shift of Schottky Barrier Heights at Strongly Pinned Metal/Germanium Interface by Inserting an Ultra-Thin Insulating Film", Jpn. J. Appl. Phys., 1, p. 051406, (2008).

[106] R. T. Tung, "Schottky barrier height-do we really understand what we measure?", J. Vac. Sci. Technol. B, 11, p. 1546, (1993).

[107] R. T. Tung, "Formation of an electric dipole at metal-semiconductor interfaces", Phys. Rev. B, 64, p. 205310, (2001).

[108] L. D. Bell, "Momentum conservation for hot electrons at the $\mathrm{Au} / \mathrm{Si}(111)$ interface observed by ballistic-electron-emission microscopy", J. Vac. Sci. Technol. A, 15, p. 1358, (1997).

[109] A. J. Stollenwerk, E. J. Spadafora, J. J. Garramone, R. J. Matyi, R. L. Moore, and V. P. LaBella, "Effect of interface band structure on hot-electron attenuation lengths in Au thin films", Phys. Rev. B, 77, p. 033416, (2008).

[110] C. R. Crowell, W. G. Spitzer, L. E. Howarth, and E. E. LaBate, "Attenuation Length Measurements of Hot Electrons in Metal Films", Phys. Rev., 127, p. 2006, (1962).

[111] E. Y. Lee and L. J. Schowalter, "Phonon scattering and quantum mechanical reflection at the Schottky barrier", J. Appl. Phys., 70, p. 2156, (1991).

[112] A. Franciosi, J. H. Weaver, and D. G. O'Neill, "Interface catalytic effect: Cr at the Si(Ill)-Au interface", Phys. Rev. B, 28, p. 4889, (1983). 